on-line at: www.actabp.pl

Review

\title{
Use of HIV as a gene transfer vector
}

\author{
Krzysztof Pluta ${ }^{1 凶}$ and Magdalena Marta Kacprzak ${ }^{2}$ \\ ${ }^{1}$ Department of Hybrid Biosystems Engineering, Institute of Biocybernetics and Biomedical Engineering \\ Polish Academy of Sciences, Warszawa, Poland; ${ }^{2}$ Genomed sp. z o.o., Warszawa, Poland
}

Received: 03 October, 2009; revised: 17 November, 2009; accepted: 19 November, 2009

available on-line: 23 November, 2009

\begin{abstract}
Despite the extensive research efforts over the past 25 years that have focused on HIV, there is still no cure for AIDS. However, tremendous progress in the understanding of the structure and biology of the HIV virus led to the development of safe and potent HIV-based transgene delivery vectors. These genetic vehicles are referred to as lentiviral vectors. They appear to be better suited for particular applications, such as transgene delivery into stem cells, compared to other viral- and non-viral vectors. This is because Lentivirus-based vectors can efficiently infect nondividing and slowly dividing cells. In the present review article, the current state of understanding of HIV-1 is discussed and the main characteristics that had an impact on vector design are outlined. A historical view on the vector concept is presented to facilitate discussion of recent results in vector engineering in a broader context. Subsequently, a state of the art overview concerning vector construction and vector production is given. This review also touches upon the subject of lentiviral vector safety and related topics that can be helpful in addressing this issue are discussed. Finally, examples of Lentivirus-based gene delivery systems and their applications are presented, with emphasis on animal transgenesis and human gene therapy.
\end{abstract}

Keywords: Lentivirus, lentiviral vectors, AIDS, HIV-1 life cycle; viral-host protein interactions, gene therapy, animal transgenesis, cell engineering

\footnotetext{
$\square$ Corresponding author: Krzysztof Pluta, Department of Hybrid Biosystems Engineering, Institute of Biocybernetics and Biomedical Engineering, Polish Academy of Sciences, Trojdena 4, 02-109 Warszawa, Poland; tel: (48) 22 659 9143; fax: (48) 22659 7030; e-mail: kpluta@ibib.waw.pl

Abbreviations: AIDS, acquired immunodeficiency syndrome; APC, antigen presenting cell; bGHpA, bovine growth hormone polyadenylation sequence; BL2, Biosafety Level 2 (containment); CA, capsid protein; CaP, DNA/calcium phosphate coprecipitation; $\mathrm{CFU} / \mathrm{ml}$, colony forming units per $\mathrm{ml}$; $\mathrm{cHS} 4$, chicken hypersensitive site 4 sequence ( $\beta$-globin insulator); $\mathrm{CMV}$, cytomegalovirus; CNS, central nervous system; $\mathrm{CPPT}$, central polypurine tract; CTS, central termination sequence; DC, dendritic cell; $\Delta \mathrm{U} 3$, self-inactivating deletion in U3 region of 3' LTR; DIS, viral RNA dimerization initiation site (dimerization signal); Dox, doxycycline; DSE, distal sequence element; EGFP, enhanced green fluorescent protein; ELISA, enzyme-linked immunosorbent assay; Env, envelope glycoprotein; FACS, fluorescence activated cell sorting; gRNA, viral genomic RNA; HBS, HEPES-buffered saline; HEK 293T, human embryonic kidney 293T cells; HIV, human immunodeficiency virus; HOS, human osteosarcoma cells; HSPC, hematopoietic stem/progenitor cell; IDLV, integration-deficient lentiviral vector; IN, viral integrase; iPS, induced pluripotent stem cell; IRES, internal ribosome entry sequence; KRAB, Krüppel-associated box (silencer domain); LEDGF/p75, lens epithelium-derived growth factor; LTR, viral long terminal repeat; MA, matrix protein; MLV, murine leukemia virus; MOI, multiplicity of infection; NC, nucleocapsid protein; NLS, nuclear localization sequence; NPC, nuclear pore complex; PBS, primer binding site; PEI, poly(ethylene imine); PIC, viral preintegration complex; polyA (pA), polyadenylation signal; PPT, polypurine tract; PR, viral protease; Pr55, Gag precursor protein; Pr160, Gag-pol precursor protein; PSE, proximal sequence element; $\psi$, viral RNA packaging signal; qRT-PCR, quantitative reverse transcriptase polymerase chain reaction; R, LTR repeat element; RCL, replication competent lentivirus; RNAP II, RNA polymerase II; RNAP III, RNA polymerase III; RRE, Rev response element; RT, reverse transcriptase; RTC, viral reverse transcription complex; rtTA, reverse Tet-controlled transactivator; SA, splice acceptor site; SD, splice donor site; shRNA, small hairpin RNA; SIN, self-inactivating (vector); siRNA, small interfering RNA; SIV, simian immunodeficiency virus; SU, gp120 surface subunit; TAR, transactivation response element; Tet, tetracycline; tetR, Tet repressor; TM, gp41 transmembrane subunit; TRE, Tet-responsive element; tTA, Tet-controlled transactivator; tTS, Tet-controlled transcriptional repressor; TU/ml, transducing units per ml; U3, LTR 3' unique element; U5, LTR 5' unique element; UTR, untranslated region; VSV-G, vesicular stomatitis virus G glycoprotein; WPRE, woodchuck hepatitis virus post-transcriptional regulatory element.
} 


\section{INTRODUCTION}

The 2008 Nobel Prize in Physiology or Medicine was shared by three clinician scientists who had worked with two important viral pathogens, human papilloma virus (HPV) and human immunodeficiency virus (HIV). Harald zur Hausen was awarded the prize "for his discovery of HPVs causing cervical cancer" and Françoise Barré-Sinoussi together with Luc Montagnier - "for their discovery of HIV". The discovery of HPV (Dürst et al., 1983) eventually led to the development of safe and effective vaccines. The first such vaccine, Silgard/Gardasil (Merck/Sanofi Pasteur MSD), was licensed in EU countries by the European Medicines Agency (EMEA) in 2006 and the second one, Cervarix (Glaxo Smith Kline, GSK), in 2007 (source: EMEA; see List of websites [1]). In contrast, over twenty five years after the link between HIV and the acquired immunodeficiency syndrome (AIDS) was demonstrated (Barré-Sinoussi et al., 1983), no efficacious vaccine for HIV is in sight (for comments see: Veljkovic et al., 2008; Amanna \& Slifka, 2009).

Although prophylactic vaccines are yet to be formulated, the intensive and detailed studies on HIV biology helped to design effective antiviral drugs, such as CCR5-receptor antagonists (Lieberman-Blum et al., 2008) or integrase inhibitors (Cocohoba \& Dong, 2008), and led to the establishment of clinical protocols, introduced in 1996, referred to as "highly active antiretroviral therapy (HAART)" (for current reviews see: Flessa \& Marschall, 2009; Bowman et al., 2009; Richman et al., 2009; for complete list of drugs used in the treatment of HIV infection, approved by the U.S. Food and Drug Administration (FDA), see List of websites [2]).

HIV and other lentiviruses (a genus of the Retroviridae family; for taxonomy details see: The Universal Virus Database (ICTVdB), authorized by the International Committee on Taxonomy of Viruses (ICTV); List of websites [3] and [4]), due to their unique features, have emerged as a powerful tool for transgene delivery into cells and for generation of transgenic animals. Moreover, HIV-based vectors have reached the clinic as promising vehicles for anti-viral gene therapy or vaccine applications.

Viral vectors have gained their popularity in basic research and gene therapy applications because of their high rates of gene transfer that are far superior to those achieved with non-viral methods. Numerous types of virus-derived gene transfer systems are available these days. These genetic vehicles are based either on DNA viruses or on RNA viruses. The corresponding vectors either integrate into the host genome or express their genetic information episomally.
Retroviral vectors, due to their ability to integrate into the host DNA, are widely used in both cell biology and biomedicine. Retrovirus-based systems (for a review see: Buchschacher, 2001), unlike episomal viruses (herpes simplex virus - HSV, adenovirus), offer stable, long-term transgene expression. Simple retroviruses (so-called gamma- or oncoretroviruses) play an important role in the modern biomedicine. More than $21 \%$ of gene therapy protocols approved worldwide until March 2009 (1537 trials) employed retroviral vectors and, among all viral and non-viral methods, only adenovirus-based vectors are currently more popular (about $24 \%$ ) (data provided by J Gene Med; see List of websites [5]).

A serious drawback of using onco-retroviruses in clinical trials is their natural disposition to integrate near promoters and regulatory regions $(\mathrm{Wu}$ et al., 2003; De Palma et al., 2005; Tsukahara et al., 2006; Deichmann et al., 2007; reviewed in Daniel \& Smith, 2008) and to induce insertional tumors due to the presence of potent transcriptional enhancers in viral long terminal repeats (LTRs). In fact, such an adverse outcome has been reported earlier in two X-linked severe combined immunodeficiency (X-SCID1) patients treated with cytokine-activated hematopoietic stem/progenitor cells (HSPCs) transduced with murine leukemia virus - MLV (trial initiated in 1999) (Hacein-Bey-Abina et al., 2003). More recently, further two cases with T cell leukemia developed after this gene therapy trial have been described. A detailed study has revealed that in all four patients blast cells harbor activating vector insertions near the LIM domain-only 2 (LMO2) and BMI1 proto-oncogenes (Hacein-Bey-Abina et al., 2008). Not surprisingly, in a more recent attempt to correct the same immune dysfunction (trial initiated in 2001), one of ten patients successfully treated has developed leukemia due to $L M O 2$ overexpression as a result of an adjacent vector integration (Board of the European Society of Gene and Cell Therapy, 2008). Also here the MLV vector was used, but in a different transduction protocol. In a Swiss-German gene therapy trial started in 2006 - correction of chronic granulomatous disease (X-CGD) - expansion of clones by insertional activation of growthpromoting genes by spleen focus-forming virus (SFFV) was observed (Ott et al., 2006). Disease complications have caused death of one of three successfully treated patients (European Society of Gene Therapy (ESGT), 2006).

Another limitation to the application of gamma-retroviral vectors is that they can infect only dividing cells. This is because viral preintegration complex (PIC), a large nucleoprotein complex responsible for viral cDNA integration, cannot enter the cell nucleus and requires disassembly of the nu- 
clear envelope during mitosis (Roe et al., 1993; Lewis \& Emerman, 1994).

These drawbacks can be avoided by using lentiviral vectors. Lentiviruses have evolved different strategies to interact with the host cell chromatin and do not integrate preferentially into close proximity of transcription start sites but rather favor introns in chromosomal regions rich in expressed genes (Schroder et al., 2002; Mitchell et al., 2004; De Palma et al., 2005; Laufs et al., 2006; Yang et al., 2008a; Felice et al., 2009; Wang et al., 2009a). This discrepancy in the integration site selection between simple retroviruses and their more complex relatives could be explained by the different interactions of host cellular factors with viral integrase (IN) and Gag-derived proteins (Lewinski et al., 2006). One of such host proteins responsible for proviral integration, lens epithelium-derived growth factor (LEDGF/ p75), interacting with HIV-1 IN, has been identified (Cherepanov et al., 2003). LEDGF accounts for the characteristic propensity of Lentivirus to integrate within active transcription units and is required for efficient viral replication (for review see: Engelman \& Cherepanov, 2008; Ciuffi, 2008).

Another advantage of Lentivirus-based vectors, important for research and gene therapy applications, is their ability to infect both dividing and nondividing cells. This feature, unique among retroviruses, substantially increases the range of cells available for gene transfer. Lentiviral vectors transduce quiescent cells, including primary hepatocytes (Pfeifer et al., 2001; Zamule et al., 2008; Dagher et al., 2009), progenitor and stem cells (Reiser et al., 1996; Miyoshi et al., 1999; Ricks et al., 2008; Santoni de Sio \& Naldini, 2009), nonproliferating monocytes and macrophages (Weinberg et al., 1991; Naldini et al., 1996a; Zufferey et al., 1997; Su et al., 2008; Veron et al., 2009) as well as postmitotic neurons (Naldini et al., 1996a; 1996b; Blömer et al., 1997; Zufferey et al., 1997; Wong et al., 2004; Hioki et al., 2009; Federici et al., 2009).

The first recombinant retroviral vector systems, with a replication-defective virus, were developed in the early 1980s (Mann et al., 1983; Watanabe \& Temin, 1983). Seven years later, and seven years after the discovery of HIV, an early system for recombinant HIV-1-based vector production was described (Page et al., 1990; Landau et al., 1991). In this system replication-defective HIV-1 vector with the sequences encoding the viral envelope (Env) glycoprotein deleted was cotransfected with expression vectors encoding heterologous Env proteins (ecotropic and amphotropic MLV Env, or human T cell leukemia virus type I - HTLV-I Env) to form HIV-1 pseudotypes. Although during the last two decades HIV-1-based vectors became accepted as the most promising gene delivery tool among lentivirus- es, also other members of the genus serve as platforms for recombinant vector generation. To date, constructs based on genomes of the following lentiviruses have been developed: human immunodeficiency virus 2 (HIV-2) (Poeschla et al., 1998a), various simian immunodeficiency viruses (SIVs) (Nègre et al., 2000; Nakajima et al., 2000), feline immunodeficiency virus (FIV) (Poeschla et al., 1998b), bovine immunodeficiency virus (BIV) (Berkowitz et al., 2001a), caprine arthritis-encephalitis virus (CAEV) (Mselli-Lakhal et al., 1998), equine infectious anemia virus (EIAV) (Olsen, 1998), Jembrana disease virus (JDV) (Metharom et al., 2000) and Maedi-Visna virus (MVV) (Berkowitz et al., 2001b).

Lentiviral vectors, due to their unique properties, including selection of "safe" integration sites in the host genome, efficient long-term gene delivery to both dividing and nondividing cells, relatively large cargo capacity $(7-8 \mathrm{~kb}$ without affecting vector titer) and target specificity achieved by pseudotyping are promising agents in research and medicine. However, their origin from mammalian pathogens, especially those developed from HIV viruses, raises the question of safety and this matter needs careful consideration.

This article presents a brief overview on the Lentivirus-host interactions and describes problems associated with the design of safe and efficacious lentiviral vectors, methods of production and purification of high titer virus preparations, strategies of delivery of genetic information to the diverse eukaryotic cells using pseudotyped lentiviruses as well as applications of recombinant lentiviral vectors in animal transgenesis and human gene therapy.

\section{HIV-1 - BASIC VIROLOGY}

\section{AIDS}

On June 5, 1981, the Morbidity and Mortality Weekly Report (MMWR) published information from the Centers for Disease Control and Prevention (CDC) about five homosexual men in Los Angeles treated for Pneumocystis carinii pneumonia (PCP) (CDC, 1981). This was the first published notice of what, a year later, became known as AIDS. Since the beginning of the epidemic, 25 million people have died of HIV-related causes. Thus, HIV is one of the leading causes of death worldwide and it leads to higher mortality than any other infectious disease.

From a clinical point of view, HIV is the most relevant Lentivirus. According to UNAIDS' 2008 Report on the global AIDS epidemic, there were an estimated 33 million (30-36 million) people living with HIV globally in 2007. The annual number of new 
HIV infections in 2007 was 2.7 million. Overall, 2 million people died due to AIDS in 2007, compared with an estimated 1.7 million in 2001 (data available from UNAIDS; see List of websites [6]).

We do not intend to review the medical conditions that are connected with AIDS in detail. Progression of HIV-related disease, epidemiology and treatment, have been recently discussed by others (Klimas et al., 2008; Wainberg \& Jeang, 2008; Weiss, 2008; Ho \& Bieniasz, 2008). Briefly, an initial, acute phase of HIV infection is followed by gradual immune exhaustion. HIV infects CD4+ lymphocytes as well as a variety of other cells, including monocytes and thymocytes, via interactions with cell surface molecules, such as CD4 receptor and chemokine coreceptors (CXCR4, CCR5). Since CD4+ cells (T helper cells) play a key role in the immune system by activating other cells such as the cytotoxic T cells and the B cells, their depletion from mucosal-associated lymphoid tissue (MALT) and blood circulation initially leads to minor infections, including cold sores (herpes simplex), condyloma (warts) and fungal infections, thrush and vaginal candidiasis, followed by serious opportunistic infections and cancers typical of AIDS.

The virulence and pathogenesis of HIV is reviewed thoroughly by Forsman and Weiss (2008). While the answer as to why HIV is a pathogen is obvious: the virus is a parasite that destroys the host immune system, there is not an easy answer as to why HIV eventually kills us. It is not clear how the high virulence of the pathogen might help in increasing its transmissibility. It is a paradox that HIV-1 is highly pathogenic in humans, whereas its precursor in chimpanzees, simian immunodeficiency virus (SIVcpz), is not. Infection of chimpanzees with HIV-1 also does not cause AIDS, so it is mostly the host rather than the virus that determines whether the disease occurs. The second human AIDS virus, HIV-2, derived from non-pathogenic SIVsm of the sooty mangabey, is less virulent in humans than HIV-1, but those individuals who develop AIDS do so with symptoms similar to HIV-1 infection. A better understanding of HIV's biology and mechanisms that govern non-pathogenic infection with SIV in chimpanzees and mangabeys might eventually lead to new approaches to treating AIDS (reviewed by: Liovat et al., 2009; Sodora et al., 2009). It is particularly important due to the fact that transmissions of new HIV strains to humans from novel hosts (gorillas) are still being reported (Plantier et al., 2009).

\section{VIRUS}

Suggestions that the virus responsible for AIDS belongs to the Lentivirinae (Lentivirus) genus of family Retroviridae appeared soon after its discov- ery, when some structural and antigenic similarities to equine infectious anemia virus (EIAV) (Montagnier et al., 1984) and sequence homology to MaediVisna virus (MVV) (Sonigo et al., 1985; Gonda et al., 1985) were reported. Lentiviruses means "slow" viruses, so named because the course of infection is characterized by a long interval between the initial infection and the onset of serious symptoms.

Lentiviruses have more complex genomes than simple retroviruses and employ sophisticated mechanisms that control all steps of infection. Some of these mechanisms rely on higher-order structures of the viral genome that, until very recently, were only poorly understood. The architecture and secondary structure of an entire HIV-1 genome, at single nucleotide resolution, has been recently published by Weeks and co-workers (Watts et al., 2009). For the first time, we can appreciate the structural complexity of the viral genomic RNA.

Lentiviral genome consists of two linear positive-sense single-stranded RNA molecules. The dimeric nature of the HIV-1 RNA genome is largely responsible for the high genetic variability of the viruses due to possible recombinations during reverse transcription (Rhodes et al., 2003). After its conversion into cDNA, in the act of viral invasion of the host cell, it becomes integrated into chromosomal DNA as a provirus (the size of the HIV-1 provirus is about $9.7 \mathrm{~kb}$; details can be found in HIV Sequence Compendium 2009 published on-line; see List of websites [7]) and is transmitted through daughter cell generations upon cell division. As an independent transcription unit, it has its own regulatory elements and is transcribed by the cell's transcription system. Figure 1A shows the structural features of HIV-1 provirus.

The protein-encoding regions are flanked by $5^{\prime}$ and $3^{\prime}$ LTRs, which consist of $3^{\prime}$ unique elements (U3), repeat elements (R) and $5^{\prime}$ unique elements (U5), and harbor some of the cis-acting elements. These cis elements contain signals important for provirus integration into the host genome (att repeats, which are located at the $5^{\prime}$ and $3^{\prime}$ ends of provirus DNA), enhancer/promoter sequences, transactivation response element (TAR) and polyadenylation signal (polyA). Besides the two LTRs there are other cisacting sequences including the primer binding site (PBS); viral RNA packaging/dimerization signals $(\psi$ and DIS); central polypurine tract (cPPT) and the central termination sequence (CTS), leading to the formation, during reverse transcription, of a threestranded DNA structure called the central DNA Flap. In addition, there is the Rev response element (RRE) that is essential for post-transcriptional transport of unspliced and incompletely spliced viral mRNAs from the nucleus to cytoplasm and the purine-rich region (polypurine tract; PPT), which pro- 


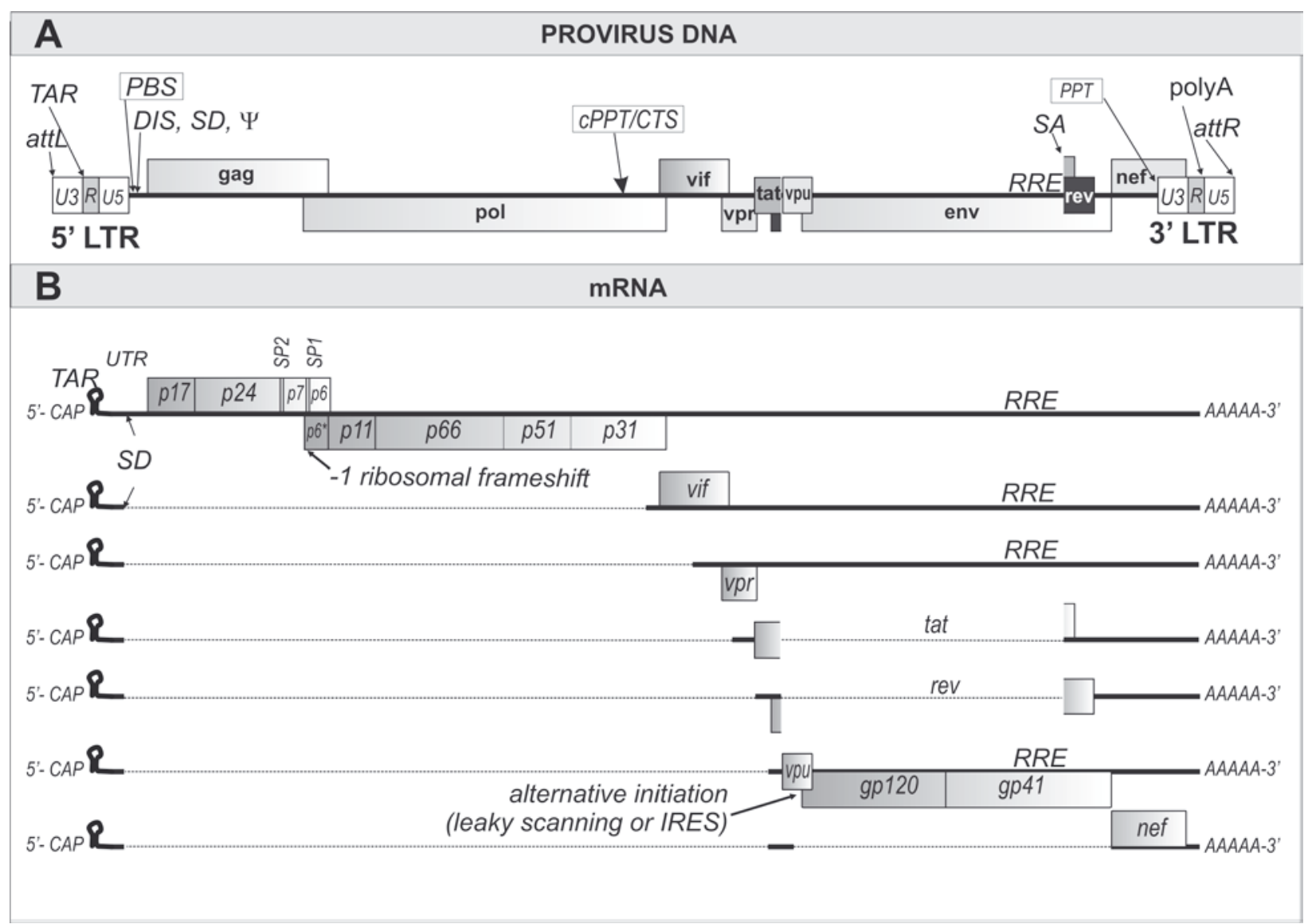

Figure 1. Schematic representation of HIV-1 genome features.

A. Elements present in the provirus. Rectangles (except LTRs) represent open reading frames positioned according to frame number $(1,2,3)$ above, on, and below the line, respectively. The cis elements crucial for replication (PBS, cPPT, CTS, PPT) and integration (attL, attR) of the virus, transcription (U3, TAR, polyA), splicing (SA, SD) and export of unspliced transcripts (RRE), for dimerization (DIS) and packaging ( $\psi$ ) of genomic RNA are indicated. B. Viral mRNA products resulting from provirus transcription and RNA splicing. Three classes of viral mRNAs are present in infected cells: approx. $9.2 \mathrm{~kb}$, approx. $4 \mathrm{~kb}$ and approx. $1.8 \mathrm{~kb}$. Rectangles represent expected final protein products that result from transcription, RNA splicing and protease cleavage of protein precursors. Ribosomal frameshift and alternative translation initiation sites are indicated.

Abbreviations: LTR, long terminal repeat; attL, attR, left and right attachment sites; U3, 3' unique element; R, repeat element; U5, 5' unique element; TAR, transactivation response element; PBS, primer binding site; DIS, dimerization signal; $\mathrm{SD}$, splice donor site; SA, splice acceptor site; $\psi$, packaging signal; cPPT, central polypurine tract; CTS, central termination sequence; RRE, Rev response element; PPT, 3' polypurine tract; polyA, polyadenylation signal; UTR, untranslated region; cap, terminal 7-methylguanosine; IRES, internal ribosome entry sequence; AAAAAA, polyA tail.

vides a second RNA primer for the initiation of plus strand DNA synthesis by virus-specific reverse transcriptase (reviewed by: Srinivasakumar, 2001; Spirin et al., 2008; for more information about structural features of HIV-1 genome see also: HIV Sequence Compendium 2009 - List of websites [7]).

The trans elements of the HIV-1 provirus include nine open reading frames (ORFs). The gag-pol, gag and env ORFs are encoding structural proteins and enzymes that are typical of all retroviruses. Additional ORFs code for essential regulatory proteins (tat and rev genes) and accessory proteins (genes: vif, $v p u, v p r$, and nef). Due to the compact size of the viral genome various strategies are used for expression of its genetic information (Fig. 1). There are nineteen protein products encoded by the provirus (summa- rized in Table 1). In almost all retroviruses, 5' LTR drives transcription of an initial genome-length RNA that also acts as an mRNA for translation of the viral Gag and Pol proteins. The Gag precursor protein (Pr55) is proteolytically cleaved by viral protease to yield the matrix (MA; p17), capsid (CA; p24), nucleocapsid (NC; p7), p6 and two spacer polypeptides: SP1 and SP2 (reviewed by Bukrinskaya, 2007). The Gag-pol precursor protein (Pr160), resulting from ribosomal slippage on a highly structured SLIP element ( -1 frameshift) into the overlapping pol ORF, is cleaved by the same protease resulting in the $p 6^{*}$ transframe (TF) polypeptide and three viral enzymes: protease (PR; p11), reverse transcriptase with ribonuclease $\mathrm{H}$ (RNase $\mathrm{H}$ ) activity (RT; heterodimer p66/p51 and RNase H; p15), and integrase (IN; p31). 
Table 1. HIV-1 proteins and their functions

\begin{tabular}{|c|c|c|c|c|}
\hline Category & Gene/Origin & Protein & Protein function & References \\
\hline \multirow{7}{*}{ 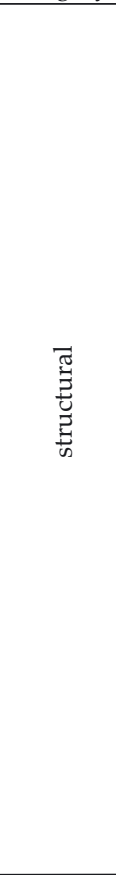 } & \multirow[t]{6}{*}{$\begin{array}{l}g a g / \\
\text { viral protease-me- } \\
\text { diated cleavage of } \\
\text { Gag polyprotein } \\
\text { (Pr55) during vi- } \\
\text { rion maturation }\end{array}$} & matrix (MA; p17) & $\begin{array}{l}\text { nuclear import of viral PIC, export of Gag- } \\
\text { RNA complex to cytosol, membrane binding, } \\
\text { virion (coats inner leaflet of viral envelope) } \\
\text { and PIC formation }\end{array}$ & $\begin{array}{l}\text { Gallay et al., 1995; 1996; reviewed } \\
\text { by: Scarlata \& Carter, 2003; Bu- } \\
\text { krinskaya, 2007; Ganser-Pornillos } \\
\text { et al., } 2008\end{array}$ \\
\hline & & capsid (CA; p24) & $\begin{array}{l}\text { membrane binding and capsid formation (core } \\
\text { shell) }\end{array}$ & $\begin{array}{l}\text { reviewed by: Scarlata \& Carter, } \\
\text { 2003; Bukrinskaya, 2007; Ganser- } \\
\text { Pornillos et al., } 2008\end{array}$ \\
\hline & & $\begin{array}{l}\text { nucleocapsid (NC; } \\
\text { p7) }\end{array}$ & $\begin{array}{l}\text { membrane binding, virion and PIC formation } \\
\text { (coats genomic RNA), RNA binding and cofac- } \\
\text { tor of RT, genomic RNA selection, packaging } \\
\text { and dimerization during virion assembly }\end{array}$ & $\begin{array}{l}\text { reviewed by: Scarlata \& Carter, } \\
\text { 2003; Bukrinskaya, 2007; Ganser- } \\
\text { Pornillos et al., 2008; Mougel et } \\
\text { al., } 2009\end{array}$ \\
\hline & & SP1 & $\begin{array}{l}\text { regulation of cleavage rate, membrane binding } \\
\text { and virion formation }\end{array}$ & $\begin{array}{l}\text { reviewed by: Bukrinskaya, 2007; } \\
\text { Ganser-Pornillos et al., } 2008\end{array}$ \\
\hline & & SP2 & $\begin{array}{l}\text { regulation of cleavage rate, membrane binding, } \\
\text { virus budding and virion formation }\end{array}$ & $\begin{array}{l}\text { reviewed by: Bukrinskaya, 2007; } \\
\text { Ganser-Pornillos et al., } 2008\end{array}$ \\
\hline & & p6 & $\begin{array}{l}\text { Vpr recruitment to virion, PTAP motif engaged } \\
\text { in interactions with host proteins, membrane } \\
\text { binding and virion formation }\end{array}$ & $\begin{array}{l}\text { Paxton et al., 1993; } \\
\text { Salgado et al., 2009; } \\
\text { reviewed by: Scarlata \& Carter, } \\
\text { 2003; Bukrinskaya, 2007; Ganser- } \\
\text { Pornillos et al., } 2008\end{array}$ \\
\hline & \multirow{4}{*}{$\begin{array}{l}\text { gag-pol/ } \\
\text { cleavage of Gag- } \\
\text { pol polyprotein } \\
\text { (Pr160) by PR } \\
\text { during virion ma- } \\
\text { turation }\end{array}$} & $\begin{array}{l}\text { p6 } 6^{*} \text { (transframe } \\
\text { polypeptide; TF) }\end{array}$ & $\begin{array}{l}\text { stabilization of Gag-Gag-pol interactions and } \\
\text { activation of PR-mediated cleavage }\end{array}$ & Chiu et al., 2006 \\
\hline \multirow{3}{*}{ 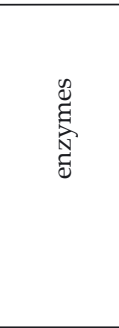 } & & $\begin{array}{l}\text { reverse transcrip- } \\
\text { tase and RNase H } \\
\text { (RT: heterodimer } \\
\text { p66/p51 and p15) } \\
\end{array}$ & $\begin{array}{l}\text { synthesis of viral cDNA (reverse transcriptase } \\
\text { enzymatic activity with ribonuclease } \mathrm{H} \text { activity } \\
\text { located at C-terminus of p66 subunit) }\end{array}$ & $\begin{array}{l}\text { reviewed by: Basu et al., 2008; } \\
\text { Mougel et al., } 2009\end{array}$ \\
\hline & & protease $(\mathrm{PR} ; \mathrm{p} 11)$ & cleavage of p160, virion maturation & $\begin{array}{l}\text { reviewed by: Boden \& Marko- } \\
\text { witz, 1998; Louis et al., } 2007\end{array}$ \\
\hline & & $\begin{array}{l}\text { integrase (IN; } \\
\text { p31) }\end{array}$ & $\begin{array}{l}\text { nuclear import of viral PIC, viral cDNA inte- } \\
\text { gration into host genome }\end{array}$ & $\begin{array}{l}\text { Gallay et al., 1997; Woodward et } \\
\text { al., 2009; reviewed by Poeschla, } \\
2008\end{array}$ \\
\hline \multirow{2}{*}{ 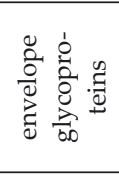 } & \multirow{2}{*}{$\begin{array}{l}\text { env/ } \\
\text { cleavage of Env } \\
\text { (gp160) protein by } \\
\text { cellular proteases }\end{array}$} & gp120 (SU) & $\begin{array}{l}\text { interaction with host cell CD4 receptor and } \\
\text { coreceptors }\end{array}$ & reviewed by Melikyan, 2008 \\
\hline & & gp41 (TM) & $\begin{array}{l}\text { anchors gp120/gp41 complex in virus membra- } \\
\text { ne, membrane fusion with host cell }\end{array}$ & $\begin{array}{l}\text { reviewed by: Weissenhorn et al., } \\
\text { 2007; Melikyan, } 2008\end{array}$ \\
\hline \multirow{2}{*}{ 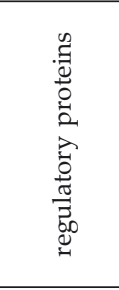 } & tat & $\begin{array}{l}\text { transactivator } \\
\text { (Tat; p16/p14) }\end{array}$ & $\begin{array}{l}\text { initiation of transcription from viral LTR, } \\
\text { transcriptional elongation, chromatin remode- } \\
\text { ling, regulation of apoptosis, modulator of host } \\
\text { immune response }\end{array}$ & $\begin{array}{l}\text { reviewed by: Seelamgari et al., } \\
\text { 2004; Barboric \& Peterlin, 2005; } \\
\text { Bolinger \& Boris-Lawrie, 2009; } \\
\text { Cheng et al., } 2009\end{array}$ \\
\hline & rev & $\begin{array}{l}\text { regulator of } \\
\text { expression of } \\
\text { viral proteins } \\
\text { (Rev; p19) } \\
\end{array}$ & $\begin{array}{l}\text { control of proviral DNA integration, stimula- } \\
\text { tion of nuclear export of unspliced HIV RNAs, } \\
\text { stimulation of translation of HIV RNAs }\end{array}$ & $\begin{array}{l}\text { Levin et al., 2009; reviewed by: } \\
\text { Cullen, 2003; Seelamgari et al., } \\
\text { 2004; Suhasini \& Reddy, 2009; } \\
\text { Groom et al., } 2009\end{array}$ \\
\hline \multirow{4}{*}{ 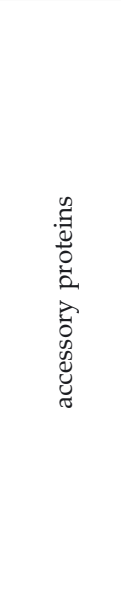 } & nef & $\begin{array}{l}\text { negative factor } \\
\text { (Nef; p27) }\end{array}$ & $\begin{array}{l}\text { down-regulation of CD4 receptor, down-regu- } \\
\text { lation of MHC-I and MHC-II, signal transduc- } \\
\text { tion interference, enhancing infectivity (actin } \\
\text { cytoskeleton rearrangement) }\end{array}$ & $\begin{array}{l}\text { Stumptner-Cuvelette et al., 2001; } \\
\text { reviewed by: Seelamgari et al., } \\
\text { 2004; Anderson \& Hope, 2005; } \\
\text { Foster \& Garcia, } 2008\end{array}$ \\
\hline & vif & $\begin{array}{l}\text { viral infectivity } \\
\text { factor (Vif; p23) }\end{array}$ & $\begin{array}{l}\text { inactivation of host immune response via bin- } \\
\text { ding apolipoprotein B mRNA editing enzyme } \\
\text { (APOBEC3 proteins) and interferon regulatory } \\
\text { factor, IRF3 }\end{array}$ & $\begin{array}{l}\text { reviewed by: Seelamgari et al., } \\
\text { 2004; Barraud et al., 2008; Oku- } \\
\text { mura et al., } 2008\end{array}$ \\
\hline & $v p r$ & $\begin{array}{l}\text { viral protein } \mathrm{R} \\
\text { (Vpr; p14) }\end{array}$ & $\begin{array}{l}\text { nuclear import of viral PIC, control of fidelity } \\
\text { of reverse-transcription, cell cycle arrest (block } \\
\text { of G2/M transition), induction of apoptosis, } \\
\text { transactivation of HIV LTR and host cell genes, } \\
\text { modulation of host immune response, inhibi- } \\
\text { tion of cellular and viral pre-mRNA splicing }\end{array}$ & $\begin{array}{l}\text { reviewed by: Seelamgari et al., } \\
\text { 2004; Le Rouzic \& Benichou, } \\
\text { 2005; Andersen et al., 2008; } \\
\text { Majumder et al., 2009; Zhang \& } \\
\text { Aida, 2009; Okumura et al., } 2008\end{array}$ \\
\hline & vpu & $\begin{array}{l}\text { viral protein U } \\
(\mathrm{Vpu} ; \mathrm{p} 16)\end{array}$ & $\begin{array}{l}\text { degradation of CD4 receptor, release of viral } \\
\text { particles, regulation of apoptosis }\end{array}$ & $\begin{array}{l}\text { Neil et al., 2008; reviewed by: } \\
\text { Seelamgari et al., 2004; Nomagu- } \\
\text { chi et al., } 2008\end{array}$ \\
\hline
\end{tabular}




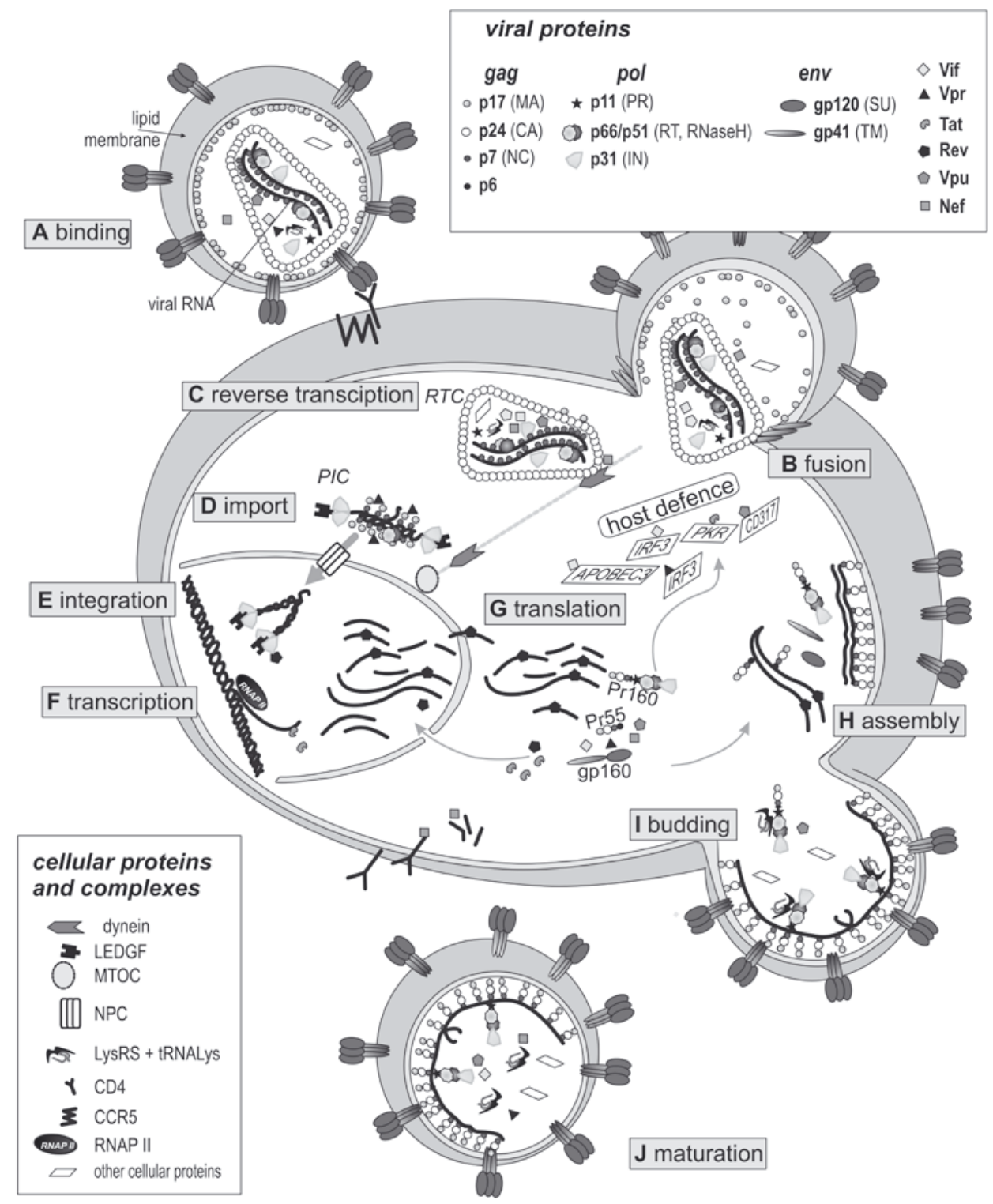

Figure 2. Scheme of HIV-1 life cycle.

Virus replication starts when the viral envelope glycoprotein gp120 binds to the CD4 receptor and a secondary receptor on the host cell surface (A). After binding to CD4, conformational changes in non-covalently associated gp41 subunit releases free energy sufficient to promote fusion of the virus particle with cell membrane. The viral core is then spilled into the cytoplasm (B). Core movement is facilitated by Nef protein which induces actin rearrangements. Reverse transcription of viral RNA occurs within CA capsid. Cellular protein dynein transports the reverse transcription complex (RTC) towards the nucleus along microtubules anchored to microtubule organizing center (MTOC) (C). When the reverse transcription is completed, provirus DNA and several viral proteins such as RT, IN, NC, Vpr, and MA, together with cellular proteins, form preintegration complex (PIC), which now can be actively transported into the nucleus via nuclear pore complex (NPC) (D). Proviral DNA is integrated into the host genome (E) by integrase, which cooperates with cellular protein LEDGF. Transcription of provirus is conducted by cellular RNAP II and enhanced by viral protein Tat. Unspliced and partially spliced transcripts are stabilized by viral protein Rev (F), which enables their export to the cytoplasm and subsequent translation $(\mathbf{G})$. Some of the synthesized viral proteins participate in combat against host defense. Full-length RNA molecules, Gag, Gag-pol and Env proteins, start to assemble new virus particles via Gag dimerization and multimerization, binding of Gag complexes to genomic viral RNA (gRNA) and association with cellular membrane. Env precursor gp160 is cleaved by cellular proteases to give gp120 and gp41 subunits that are directed to the cell membrane by Gag $(\mathbf{H})$. Initially, virus particles are released $(\mathbf{I})$ from infected cell as an immature form $(\mathbf{J})$. Digestion of polyproteins by viral protease leads to virus maturation (A).

Abbreviations: MA/p17, matrix protein; CA/p24, capsid protein; NC/p7, nucleocapsid protein; p6, viral p6 protein; PR/ p11, protease; RT/p66/p51, reverse transcriptase with RNase H activity; IN/p31, integrase; SU/gp120, surface glycoprotein; TM/gp41, transmembrane glycoprotein; Tat, Rev, regulatory proteins; Vif, Vpr, Vpu, Nef, accessory proteins; Pr160, Gag-pol precursor polyprotein; Pr55, Gag precursor polyprotein; gp160, envelope (Env) glycoprotein; RNAP II, RNA polymerase II. 
In more complex retroviruses including lentiviruses, this initial transcript is also processed into fully spliced transcripts encoding the Tat and Rev regulatory proteins as well as the auxiliary protein Nef. Alternatively, in HIV-1 this transcript can be processed into partially spliced mRNAs coding for the three other accessory proteins: Vif, Vpu and Vpr (Vpx, a Vpr homolog present in HIV-2 and some SIVs is absent in HIV-1). These partially processed transcripts also contain the env ORF for envelope glycoprotein (Env, gp160) translated thanks to the leaky scanning through vpu AUG. During maturation of the virus particle, cell proteases cleave the gp160 precursor to yield the gp120 surface subunit (SU) and the gp41 transmembrane subunit (TM) (reviewed by: Cullen, 2003; 2009; Bolinger \& BorisLawrie, 2009).

The lipid-enveloped HIV virion measures approximately $80-120 \mathrm{~nm}$ in diameter and contains a number of host cellular proteins important for virus replication and pathogenesis. As many as 253 proteins have been found in HIV-1 virion preparations from infected cells (Chertova et al., 2006; Saphire et al., 2006; for review see: Kolegraff et al., 2006; Ott, 2008). Figure 2A depicts the structural organization of HIV-1 particle.

\section{HIV-1 LIFE CYCLE}

The key to understanding the course of HIV infection and the Lentivirus life cycle in general is the unraveling of the virus-host protein interactions. HIV-1 genome, like those of all viruses, is too small to encode all the components necessary for its replication, therefore the virus must "hijack" the host cell machinery in order to complete its life cycle. Recently, the Division of AIDS of the National Institute of Allergy and Infectious Diseases, in collaboration with Southern Research Institute and the National Center for Biotechnology Information (NCBI), has initiated the development of an HIV-1, Human Protein Interaction Database (see List of websites [8]). Thirty-two hundred papers published between 1984 and 2007, describing putative interactions between HIV-1 and human proteins were identified by PubMed queries. A total of 1448 human proteins that interact with HIV-1, comprising 2589 unique HIV-1-to-human protein interactions, were found. Two HIV proteins stand out due to their most complex interplay with the host factors: surface glycoprotein gp120 and regulatory protein Tat (532 and 775 interactions, respectively) (Ptak et al., 2008; Fu et al., 2009).

The HIV-1 life cycle has been thoroughly described by others (Freed, 2001; Finzi et al., 2007a; Adamson \& Freed, 2007; Spirin et al., 2008; Klimas et al., 2008). Figure 2 summarizes our current knowledge about the HIV-1 replication phases. Here we focus on the crucial virus-host protein-protein interactions and the viral genome landmarks that determine individual steps of viral infection.

Entry

The HIV replication cycle starts (early phase of infection) when the viral envelope glycoprotein gp120 binds to the CD4 receptor exposed on the surface of the host CD4-positive cells (Fig. 2A) (Dalgleish et al., 1984; Landau et al., 1988). This binding triggers a series of events that lead to the ultimate destruction of those cells. The gp120-CD4 interactions may contribute to the depletion of CD4+ T cells through an interferon (IFN) $\alpha / \beta$-induced, TRAIL (tumor necrosis-related apoptosis-inducing ligand)-mediated apoptotic mechanism (Herbeuval et al., 2005; Herbeuval \& Shearer, 2007). Recently, it has also been shown that anti-CD4 antibodies prevent HIVinduced indoleamine 2,3-dioxygenase (IDO) expression in plasmacytoid dendritic cells (pDCs) (Boasso et al., 2007). IDO is an immunosuppressive enzyme that inhibits $\mathrm{T}$ cell proliferation. It was proposed that $\mathrm{HIV}$-activated CD4+ pDCs, via production of type I IFN and IDO, may exert suppressive and cytotoxic effects on T cells (Boasso \& Shearer, 2008; Boasso et al., 2008). Interestingly, gp120 alone, when incubated with human $\mathrm{T}$ cells, can affect CD4+ $\mathrm{T}$ cell migration. An increased lymph node accumulation of CD4+ $\mathrm{T}$ cells observed in HIV+ patients, with concurrent decreases in blood and spleen, could be a reason of HIV-related lymphadenopathy and lymphopenia (Green et al., 2009). Additionally, HIV-1 negative factor Nef inhibits $\mathrm{T}$ lymphocytes chemotaxis and diminishes adhesion and polarization of the $\mathrm{T}$ cells. Recent results suggest that membrane localization of the Nef and intracellular signaling events might contribute to these inhibitory effects (Park \& He, 2009).

After binding to CD4, activated gp120 undergoes a conformational change which produces a binding site for a secondary host cell receptor (Sattentau \& Moore, 1991). The most common strains of HIV-1 utilize a $\beta$-chemokine receptor, CCR5, or an $\alpha$ chemokine receptor, CXCR4, as coreceptors (Alkhatib et al., 1996; Feng et al., 1996). HIVs that use only the CCR5 receptor are termed R5, those that only use CXCR4 are termed X4, and those that use both, $X 4 R 5$. Infections with $X 4$ and R5 strains differ in their effects exerted on host cells. It has been shown recently that $\mathrm{R} 5$ viruses modulate, to a greater extent than $\mathrm{X} 4$ viruses, the level of mRNA of the resting CD4+ T cells. R5 viruses, but not X4 strains, are able to modulate $\mathrm{T}$ cell receptor (TCR)-mediated actin polymerization and signaling. Additionally, a number of genes induced exclusively by R5 envelopes were also up-regulated in the resting CD4+ T cell population, which might facilitate replication of HIV in the pool of resting CD4+ T cells (Cicala et al., 2006; Sir- 
ois et al., 2008). R5-infected cells have a higher rate of activation and proliferation (Locher et al., 2005) and $\mathrm{R} 5$ viruses produce more progeny in infected CD4+ $\mathrm{T}$ cells than do X4 strains (Schweighardt et al., 2004). As other G-protein coupled receptors, CCR5 and CXCR4 have a seven transmembrane structure. The N-terminal portion is extracellular and participates in the binding of the chemokine, whereas the C-terminus is intracellular and interacts with $\beta$-arrestin. In addition to binding the chemokine receptor to clathrin for endocytosis and recycling, $\beta$-arrestins facilitate G-protein-independent cell signaling resulting in stimulation of protein synthesis (Zhao et al., 1998; Oppermann, 2004; Signoret et al., 2005; DeWire et al., 2008). The association of CD4 with gp120 becomes unstable, once gp120 begins to bind with CCR5, giving a tighter gp120-CCR5/CD4 complex (Chang et al., 2005; Dobrowsky et al., 2008). Identifying CCR5 as the coreceptor for the majority of transmissible strains of HIV has led to the development of entry inhibitors (reviewed by Dhami et al., 2009). Moreover, people homozygous for a 32-bp deletion in this coreceptor gene are genetically resistant to infection by HIV (Liu et al., 1996; recently reviewed by Piacentini et al., 2009).

Formation of the gp120-coreceptor/CD4 complexes triggers refolding of the non-covalently associated transmembrane gp41 protein. It is not clear whether disassociation of gp120 is required for further steps of infection, but there is evidence that the interactions between gp120 and gp41 must weaken in order to initiate fusion (Abrahamyan et al., 2003). Activated gp41 protein exposes an $\mathrm{N}$-terminal hydrophobic fusion peptide inserted into the cellular plasma membrane. The heptad repeat regions (HR1 and HR2) of the gp41 trimer subsequently fold in to form a six-helix bundle (6HB or coiled-coil complex). The formation of this complex couples the viral and cellular membranes (creation of so-called fusion pore) and releases free energy sufficient to promote their fusion (Fig. 2B) (Melikyan et al., 2000; Markosyan et al., 2003; reviewed by Melikyan, 2008).

Once the fusion is initiated, the viral core contents are released into the cytoplasm. It is postulated that HIV-1 Nef induces actin rearrangements, thereby reinforcing the core's movement and penetration of the cortical actin barrier underlying the plasma membrane after fusion (Campbell et al., 2004). This requirement of Nef is avoidable when the genuine HIV Env is replaced (pseudotyped virus) with $\mathrm{pH}-$ dependent envelope glycoproteins derived from, for example, vesicular stomatitis virus (VSV-G), which enters cells via endocytosis with subsequent endosome acidification releasing the virion core (Chazal et al., 2001). However, a more recent study has shown that the infectivity stimulation by Nef is HIV-1 Env-independent and is rather attributed to virus interactions with cellular receptors (Pizzato et al., 2008). Thus, the Nef involvement in the early stages of HIV-1 entry is still controversial and other recent results call into question its importance during virion release into the cytoplasm, pointing out to its role in the biogenesis of viral particles instead (Laguette et al., 2009).

Despite the fact that the actin cytoskeleton may act as a barrier to incoming viruses at the plasma membrane it also plays a positive role in early retroviral trafficking (Bukrinskaya et al., 1998; Komano et al., 2004). The dependence of lentiviral infection on actin polymerization suggests that the actin network facilitates short-range motion of the viral cores to another region of the peripheral cytoplasm and subsequently links them to microtubules for efficient transport to the nucleus.

The fine mechanism mediating the intracellular transport of the virus capsid is poorly understood and is only known to involve the microtubule system. Live cell observation of viral trafficking, using fluorescently labeled tubulin, allowed visualizing of the movement of individual viral cores along microtubules (McDonald et al., 2002). This trafficking required a minus-end microtubule motor, dynein. Dyneins move cargo toward the minus end of microtubules, which is anchored at the microtubule organizing center (MTOC) or centrosome, in a process that is called retrograde transport (Fig. 2C). In another study, HIV-1 complexes with fluorescently labeled viral integrase showed directed movement toward the nuclear compartment, kinetically characteristic of both microtubule- and actin-dependent transport (Arhel et al., 2006).

\section{Host defense}

Viral replication depends on host cell factors and species-specific characteristics of these proteins affect viral tropism. On the other hand, evolution has equipped cells with factors that can directly inhibit retrovirus replication. This cellular anti-viral system, mediated by so-called restriction factors, is termed intrinsic immunity as distinct from conventional innate and adaptive immunity, which engage a number of genes controlling expression of antiviral cytokines (interferons - IFNs) (reviewed by: Akira et al., 2006; Kang \& Compans, 2009). To combat the host defense, some HIV-1 auxiliary gene products counteract these host inhibitors and, thus, no human protein can effectively block replication of wild type HIV-1 under physiological conditions. Understanding of this intricate interplay between host defense system, the species-specific barriers, and the viral infectivity factors is key in the ongoing search for new potent antiviral drugs. Recently, high-throughput methods have been used to identify factors that might be involved in antiviral immunity (Valente \& Goff, 2009). 
Currently, the most intensively studied host restriction proteins are: tripartite motif protein $5 \alpha$ (TRIM5 $\alpha$ ), cyclophilin A (CypA), apolipoprotein B mRNA-editing catalytic polypeptide 3 (APOBEC3), zinc finger antiviral protein 2 (ZAP2), eukaryotic initiation factor 3 subunit $\mathrm{f}$ (eIF3f) (Valente et al., 2009), heterogeneous nuclear ribonucleoprotein $\mathrm{U}$ (hnRNP $\mathrm{U})$, and cell surface protein tetherin/CD317. In the context of host defense, the following viral proteins are key players: viral infectivity factor (Vif), viral protein $\mathrm{U}(\mathrm{Vpu})$, and viral protein $\mathrm{R}(\mathrm{Vpr})$ (recently reviewed by: Towers, 2007; Wolf \& Goff, 2008; Takeuchi \& Matano, 2008; Huthoff \& Towers, 2008; Aguiar \& Peterlin, 2008; Goila-Gaur \& Strebel, 2008; Malim, 2009). Here, we will describe viral proteins that interact with cellular factors in order to abrogate restriction.

APOBEC3G (catalytic polypeptide 3G or A3G), a member of the family of cellular polynucleotide cytidine deaminases, was first identified as a cellular factor that protects human cells from infection by HIV-1 viruses lacking a vif gene (HIV-1 $\Delta$ vif) (Sheehy et al., 2002). A3G together with another family member, A3F, in the absence of Vif are encapsidated by budding virus particles and lead to excessive cytidine (C) to uridine (U) editing of negative sense reverse transcripts in newly infected cells. The $C$ to $U$ editing results in guanine $(\mathrm{G})$ to adenine (A) substitutions in the positive sense DNA strand as reverse transcription is completed (Harris et al., 2003). However, it seems that the main antiviral effects of APOBEC 3 proteins are achieved by an editing-independent mechanism: inhibition of viral DNA synthesis by impeding the translocation of reverse transcriptase along template RNA (Anderson \& Hope, 2008; Bishop et al., 2008). It is possible that to perform this action A3G/A3F needs another cellular cofactor, since not all cell lines are resistant to HIV-1 1 vif virus infection (Han et al., 2008). In physiological conditions, Vif binds to A3G, A3F, and $\mathrm{A} 3 \mathrm{C}$ and targets these proteins for polyubiquitination by forming an E3 ubiquitin ligase with cullin 5 (Cul5 - E3) (Yu et al., 2003a; Liu et al., 2005; Kobayashi et al., 2005; Zhang et al., 2008). It is also possible that Vif can inactivate the cellular restriction factors without their degradation (Kao et al., 2007; Ao et al., 2008). The ability of Vif to block the antiviral activity of APOBEC 3 is species-specific and this specificity might be a barrier for interspecies virus transfers (Simon et al., 1998; Mariani et al., 2003; Schröfelbauer et al., 2006). Recent identification of the binding sites responsible for this intriguing phenomenon may lead to generation of pharmacologic agents that would inter-

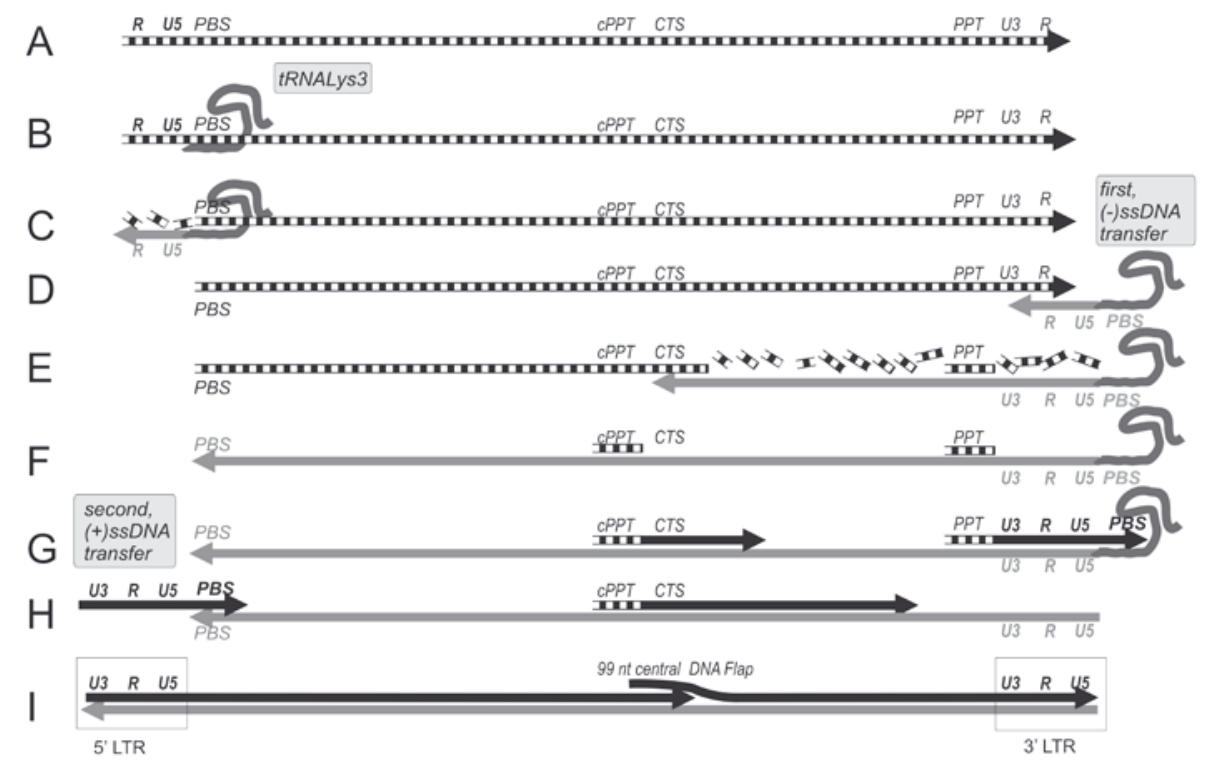

Figure 3. Reverse transcription.

Replication of lentiviral genomic RNA (gRNA) (A) is conducted by viral reverse transcriptase (RT). The process starts by binding of tRNALys primer to the primer binding site (PBS) (B). The primer is extended until the end of gRNA molecule, and RNA from the RNA/DNA hybrid is digested by RNase H subunit of RT. Resulting (-) single-stranded DNA called strong stop DNA, (-)ssDNA, is transferred to the $3^{\prime}$ end of gRNA and annealed via R region in a process called first (-) strong stop DNA transfer (D). This initiates the synthesis of the entire (-) DNA strand of the provirus (E). Simultaneous digestion of gRNA spares polypurine tracks, central polypurine track (cPPT) and 3' polypurine track $(\mathrm{PPT})(\mathbf{F})$ which in turn serve as primers for $(+)$ DNA strand synthesis $(\mathbf{G})$. When the 3' LTR, together with PBS region, is transcribed from PPT primer, the second $(+)$ strong stop DNA transfer occurs. The created $(+)$ single stranded DNA fragment is annealed to the $5^{\prime}(-)$ DNA strand via PBS region $(\mathbf{H})$. This enables reconstruction of $5^{\prime}$ LTR and synthesis of $(+)$ DNA strand up to the center of the genome. In the CTS region DNA synthesis is ended and central DNA Flap is formed (I). Dotted line represents gRNA; grey line: (-) DNA strand; black line: (+) DNA strand. Arrows indicate 5' $\rightarrow 3^{\prime}$ orientation and direction of DNA synthesis. 
fere with the interactions between Vif and APOBEC3 proteins and bring about efficient inhibition of viral replication (He et al., 2008; Pery et al., 2009).

HIV-1, like other RNA viruses, has also developed a specific mechanism to downregulate the early antiviral response. It has been shown that viral accessory proteins Vif and Vpr can independently target an interferon regulatory factor, IRF-3, for ubiquitin-mediated degradation (Okumura et al., 2008).

Another example of how lentiviruses counteract the innate host defense is the blocking of activation of the interferon-induced serine/threonine protein kinase $\mathrm{R}$ (PKR) pathway by the viral protein Tat. Tat interferes with autophosporylation of PKR or acts as a competing substrate (reviewed by Bolinger \& Boris-Lawrie, 2009). It has also been demonstrated recently that Tat impairs the interferon gamma (IFN $\gamma$ )-receptor signaling pathway by inducing the activity of suppressor of cytokine signaling-2 (SOCS-2) (Cheng et al., 2009).

Recently, an inhibitor of HIV-1 particle release termed tetherin (CD317), whose activity is neutralized by the HIV-1 accessory protein $\mathrm{Vpu}$, has been discovered (Neil et al., 2008). CD317, an interferoninduced membrane protein, causes retention of virions on the cell surface and, after endocytosis, in CD317-positive compartments. Vpu co-localizes with this protein and inhibits these effects. Interestingly, Vpu activity is species specific and HIV-1 Vpu protein has evolved to target only the transmembrane domains of human and chimpanzee tetherins $(\mathrm{McN}-$ att et al., 2009).

\section{Reverse transcription}

In the cytoplasm, virions undergo structural alterations giving rise to a large ribonucleoprotein structure called the reverse transcription complex (RTC), the site of extensive viral DNA synthesis (Fig. 2C). In vitro studies have shown that viral RNA is partly released from the core upon reverse transcription initiation and is used as a template for the RT to synthesize provirus DNA (Auewarakul et al., 2005). Yet in vivo, this synthesis takes place rather in an intact capsid (Arhel et al., 2007).

Reverse transcription requires a specific cellular tRNA annealed to the primer binding site (PBS) for the initiation of cDNA synthesis. In the case of HIV-1, tRNALys3 is preferentially selected during virus assembly. It is incorporated into the virion via interactions of the RT domain of Gag-pol with Gag and lysyl-tRNA synthetase (LysRS) (Barat et al., 1989; Saadatmand et al., 2008; Bilbille et al., 2009). After annealing of the primer and cDNA synthesis initiation, reverse transcription involves two obligatory DNA strand transfers to carry out the synthesis of the complete double-stranded proviral DNA
(dsDNA) with duplicated long terminal repeat (LTR) ends (Fig. 3). Transcription of the plus strand RNA to minus strand DNA requires a transfer of the growing DNA strand from one end of the genomic RNA (gRNA) to the other. This step is called "minus strong-stop DNA" transfer. Subsequent synthesis of the second (plus) DNA strand involves priming from cPPT and 3' PPT followed by the second strand transfer. Synthesis of the plus DNA strand continues until it reaches CTS, which leads to the creation of central DNA Flap. A basic feature of the strand transfer mechanism is the use of RNase $\mathrm{H}$, another activity of RT, to remove fragments of the RNA template from the growing DNA strand (with the exception of the polypurine tracts of cPPT and PPT), enabling a single stranded DNA region to anneal to this second site (reviewed by Basu et al., 2008). These processes are accompanied by nucleocapsid protein, NC, a potent nucleic acid chaperone, which tightly binds nucleic acids and facilitates the annealing of complementary sequences as well as the strand transfer and exchange reactions (Guo et al., 2000; reviewed by: Basu et al., 2008; Mougel et al., 2009).

The nascent viral DNA binds several viral and cellular proteins to produce so-called preintegration complex (PIC) (Fig. 2D).

\section{Nuclear import}

As mentioned before, lentiviruses, unlike simple retroviruses, are able to infect nondividing cells. This intriguing phenomenon can be explained by the fact that lentiviral PICs with the size of $56 \mathrm{~nm}$ (Miller et al., 1997) are actively transported through nuclear pore complexes (NPCs) in an ATP-dependent manner (Bukrinsky et al., 1992). As shown earlier, cargoes much larger than the assumed functional NPC diameter of $26 \mathrm{~nm}$ must be imported into the karyoplasm via an active transport mechanism (Panté \& Kann, 2002). Both viral and cellular elements take part in this process. In contrast to the HIV-1 PIC, during infection of the dividing cells by a simple Moloney murine leukemia virus (MLV) the reverse transcriptase complex/preintegration complex (RTC/PIC) retained a significant portion of capsid protein (CA) (Fassati \& Goff, 1999). Moreover, the MLV CA appears to be the dominant negative regulator of nuclear import (Yamashita \& Emerman, 2004). Since HIV-1 mutants that do not shed enough p24 CA are defective in nuclear import and integration, it has been proposed that appropriate shedding of CA protein from the lentiviral RTC/PIC is a key step for the RTC-to-PIC transition and subsequent nuclear import of HIV-1 (Dismuke \& Aiken, 2006).

According to this view, blocks in reverse transcription and in RTC maturation might be one of the reasons why lentiviruses cannot infect naïve $T$ 
lymphocytes in vitro (Zack et al., 1990; Auewarakul et al., 2005). It was recently shown, that CA-containing sub-viral complexes accumulated at centrosomes of human primary quiescent $\mathrm{CD} 4+\mathrm{T}$ cells, were stably persisting there for weeks and could be induced to replicate upon cell activation. This might reflect strategy that has evolved for the virus to persist latently in the vicinity of this organelle in resting cells and to be activated in response to cell activating stimuli (Zamborlini et al., 2007). It has long been known that in AIDS patients quiescent T lymphocytes constitute an inducible reservoir of latent HIV-1 viruses (Bukrinsky et al., 1991). Yet more recently, it was convincingly demonstrated that HIV-1 could replicate in these cells in vivo (Eckstein et al., 2001) and that also in vitro a tiny fraction of the virus population could integrate into the host genome in sites similar to those selected in prestimulated cells (Vatakis et al., 2009). However, the integration process in quiescent $\mathrm{T}$ cells is hindered by the nuclear accumulation of large numbers of incorrectly processed viral ends and abortive single- and double-LTR (1- and 2-LTR) circles that are common dead-end by-products of viral replication.

Mature HIV-1 PICs contain provirus DNA and proteins such as reverse transcriptase (RT), integrase (IN), nucleocapsid (NC), viral protein $\mathrm{R}$ (Vpr), and matrix (MA) (Fig. 2D) (Bukrinsky et al., 1993a), although the presence of the NC has been questioned (Miller et al., 1997) and disassociation of RT, once cDNA synthesis in RTC is completed has been postulated (Bukrinsky, 2004). MA and IN have functional nuclear localization sequences (NLSs) that resemble the canonical NLS of the simian virus 40 (SV40) $\mathrm{T}$ antigen and both utilize the classical nuclear import pathway dependent on importins $\alpha$ and $\beta$ (karyopherins) (Bukrinsky et al., 1993b; Gallay et al., 1996; 1997). HIV-1 also exploits importin 7, a member of importin $\beta$ family, to maximize nuclear import of its PICs via interaction with IN (Ao et al., 2007). However, this is not true for the HIV-2, SIVmac or EIAV integrases. This suggests that the closely related lentiviruses may have evolved different and probably redundant nuclear import mechanisms (Zaitseva et al., 2009).

In another recent study, HIV-1 IN was shown to directly interact with NPC via binding to nucleoporin NUP153. Overexpression of NUP153 C-terminal domain in cultured cells reduced the infectivity of an HIV-based vector by interfering with the nuclear import of the viral PIC (Woodward et al., 2009).

Also the Vpr C-terminus contains several arginine residues that resemble a basic NLS, but this sequence does not function as an NLS. Nonetheless, it was shown that Vpr associates with importin $\alpha$, and that this association results in an increased binding affinity of the basic-type NLSs, including that of MA (Popov et al., 1998; Nitahara-Kasahara et al., 2007).

An additional viral element implicated in HIV-1 nuclear import is the central polypurine tract (cPPT). Located within pol gene the cPPT is the second origin of DNA plus strand synthesis that, after completion of reverse transcription, results in a short, approximately $100 \mathrm{nt}$-long, stretch of triple-stranded DNA (so-called central DNA Flap) (Charneau et al., 1992) (Fig. 3; for details see section on Reverse Transcription). When cPPT is mutated, Flap-defective linear DNA accumulates in close proximity of the nuclear membrane, indicating a late defect in nuclear import (Zennou et al., 2000). On the other hand, insertion of the cPPT into HIV-based vectors strongly stimulates gene transfer in target cells (for references see section on CPPT-CTS below). The mechanism of action employed by this cis-acting element was unravelled by Charneau's group. They showed that maturation of RTC into PIC, followed by uncoating of the preintegration complex at the nuclear pore, is impaired in the absence of DNA Flap formation, consequently leading to the trapping of HIV-1 PIC within an integral capsid shell and subsequently prohibiting nuclear entry of the viral genome (Arhel et al., 2007). According to those observations, reverse transcription proceeds within an intact CA shell, independently of the RTC movement toward the nuclear membrane. Hence, in vivo, uncoating does not occur immediately after virus entry and reverse transcription initiation (as was observed in vitro: Auewarakul et al., 2005), but starts at the nuclear pore upon completion of cDNA synthesis and Flap forma-

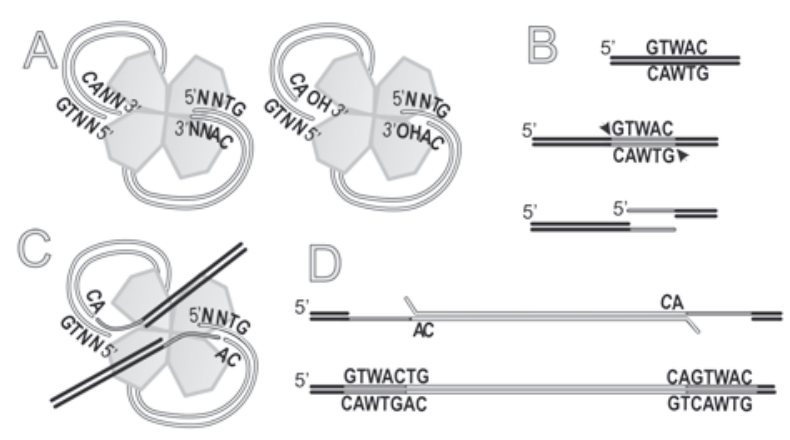

Figure 4. Scheme of provirus integration.

Integration of provirus to the host genome DNA is performed by lentiviral integrase (IN). The enzyme recognizes $5^{\prime}$ and $3^{\prime}$ short terminal fragments called att, binds and bends the DNA molecule, removes two nucleotides from each $3^{\prime}$ end (staggered cut) and exposes $\mathrm{OH}$ group (3' processing) (A). IN cleaves host genomic DNA, creating five-nucleotide-long overhangs (B), which then are bound to the free $3^{\prime} \mathrm{OH}$ groups of the provirus (DNA strand transfer) (C). The last step of integration comprises DNA repair of five-nucleotide gaps at both sides of proviral DNA by cellular enzymes (D). IN is shown in tetrameric form. GTWAC-HIV-1 target site consensus sequence (W means $\mathrm{A}$ or $\mathrm{T})$. 
tion (Fig. 2D) (Arhel et al., 2007). It was recently postulated that the additional initiation of plus strand DNA synthesis from the cPPT RNA primer facilitates the efficient completion of proviral DNA synthesis in viruses harboring kinetically impaired RT, because the enzyme needs only to synthesize half of the genome during plus strand synthesis (Skasko \& Kim, 2008).

Also other factors might be involved in the HIV-1 DNA entry to the nucleus, e.g., tRNA (Zaitseva et al., 2006). On the other hand, numerous results concerning this issue are controversial or conflicting. For example, participation of the MA and IN proteins in HIV-1 genome nuclear import, as well as implication of the central DNA Flap in this process, are still a matter of debate (Freed et al., 1997; Limon et al., 2002a; 2002b; Dvorin et al., 2002). Thus, the mechanisms of lentiviral PIC nuclear import, a critical step in the virus life cycle, need to be further investigated.

\section{Integration}

In the nucleus, the linear provirus cDNA integrates into the DNA of the host cell (Fig. 2E). Viral integrase (IN) circularizes the provirus at the att repeats and plays a crucial role in its integration into host cell DNA via recombination between the att repeats and the integration site (Fig. 4). It was shown that IN is the principal viral determinant of integration specificity (Lewinski et al., 2006). Wu et al. (2005) and Holman and Coffin (2005) have examined a large number of integration site sequences from various retroviruses, including HIV-1, and found that they are palindromic in nature. The symmetry of the target site sequence is a consequence of the fact that the IN forms symmetrical dimers, tetramers, or oligomers and that each half complex would have a similar preference for target DNA structure.

IN catalyzes two spatially and temporally distinct reactions. During the first reaction, called $3^{\prime}$ processing, IN hydrolyzes two nucleotides from both $3^{\prime}$ ends of HIV-1 DNA. The second reaction, DNA strand transfer, takes place at the site of integration. IN uses the recessed $3^{\prime}-\mathrm{OH}$ groups created during $3^{\prime}$ processing to cleave the phosphodiester backbone of chromosomal DNA in a staggered fashion, concomitantly connecting the viral DNA 3 ' ends to the generated $5^{\prime}$ overhangs by transesterification mechanism. The resultant DNA recombination intermediate harbors single-strand discontinuities and the process is completed by the third main step, gap repair by host cell enzymes. As a result of the integration a duplication of 5 bp-long fragment of host DNA flanking the provirus is created. The consensus sequence at the HIV-1 palindromic duplicated target sites is $\mathrm{G}_{1} \mathrm{~T}_{2}(\mathrm{~A} / \mathrm{T})_{3} \mathrm{~A}_{4} \mathrm{C}_{5}$ (Wu et al., 2005; Holman \& Coffin, 2005) (for recent reviews on HIV-1 integrase see: Vandegraaff \& Engelman, 2007; Delelis et al., 2008; Poeschla, 2008).

Although purified retroviral IN enzyme displays $3^{\prime}$ processing and DNA strand transfer activities in vitro (Bushman et al., 1990; Li \& Craigie, 2009), in vivo it acts in the context of PIC and numerous studies indicate that other proteins play important auxiliary roles in modulation of its activity. In HIV-infected cells, the regulator of viral protein expression Rev may be involved in the integration of proviral DNA by regulating the activity of the integrase. It has recently been shown that these two proteins interact with each other in vitro and in vivo and that Rev-derived peptides can inhibit the 3 '-end processing and strand-transfer enzymatic activities of IN in vitro (Rosenbluh et al., 2007). On the other hand, IN-derived peptides exert an opposite effect stimulating IN activity and multiple viral DNA integration events due to displacement of the Rev-derived inhibitory molecules (Levin et al., 2009). This suggests that lentiviruses have evolved mechanisms to limit the frequency of integration events in the infected cell. Transcriptionally active unintegrated HIV-1 genomes present in the infected cells can participate in HIV-1 replication and contribute to its genetic diversification and evolution (Gelderblom et al., 2008).

Among the cellular proteins that have been shown to affect lentiviral integration, recent attention has focused on LEDGF/p75 (Maertens et al., 2003). LEDGF/p75 and HRP2, members of the hepatomaderived growth factor (HDGF)-related protein (HRP) family, bind directly to IN within the viral PIC and this interaction is exclusive to lentiviruses (Llano et al., 2004; Busschots et al., 2005; Cherepanov, 2007). LEDGF/p75 is a ubiquitous nuclear protein, tightly associated with chromatin through three conserved sequence elements within the N-terminal half of the protein (N-terminal domain ensemble, NDE): the PWWP domain, NLS, and a dual copy of the AThook DNA binding motif (Llano et al., 2006; Shun et al., 2008). The C-terminal integrase-binding domain (IBD) interacts with the viral IN catalytic core domain (CCD) (Cherepanov et al., 2004; Busschots et al., 2007; Hombrouck et al., 2007). The cellular functions of LEDGF/p75 are probably involved in the survival response to environmental stress, and alternatively spliced LEDGF gene (PSIP1) products yield two coactivators of transcription: p75 and p52 (Ge et al., 1998; Singh et al., 2000; Nishizawa et al., 2001). For lentiviruses, LEDGF/p75 serves as an infectivity factor and a key determinant of the preferential integration target site selection within transcriptional units, likely acting as a receptor or molecular tether (Fig. 2E) (Ciuffi et al., 2005; Shun et al., 2007; Marshall et al., 2007; Hombrouck et al., 2007). However, some recent results suggest that chromatin tethering 

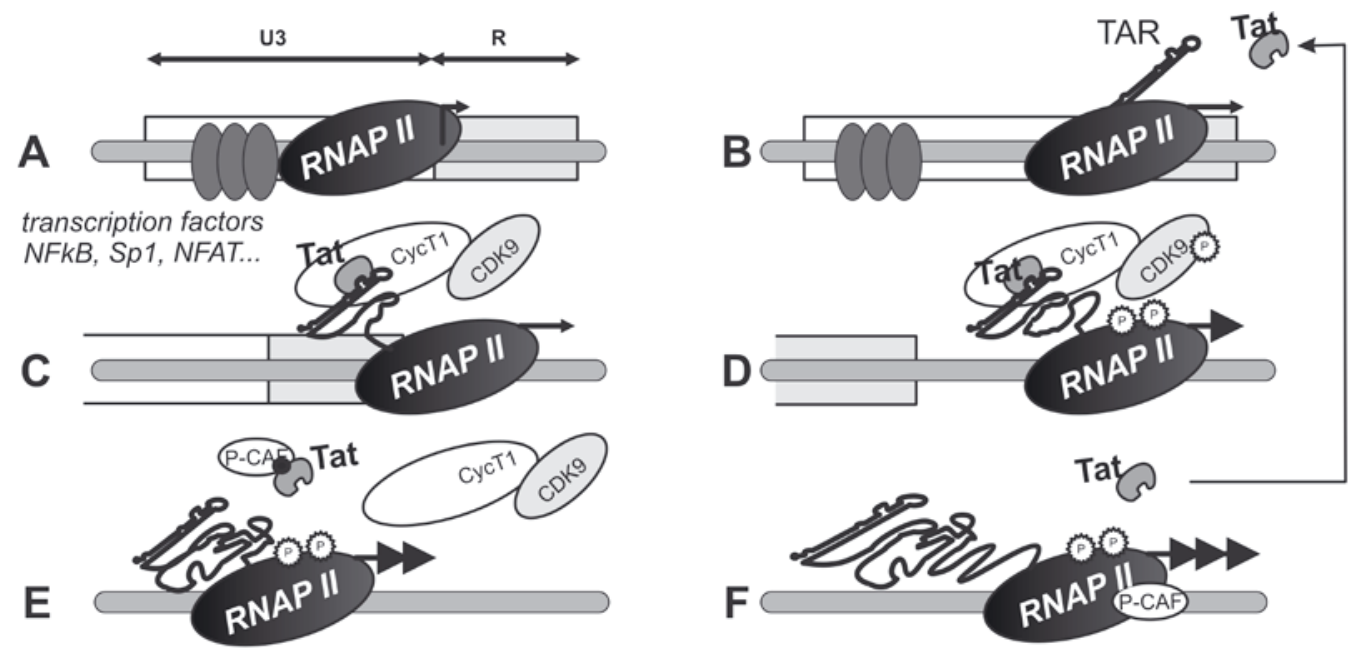

Figure 5. HIV-1 transcription initiation and elongation.

U3 region of $5^{\prime}$ LTR contains sites recognized by various cellular transcription factors. RNAP II-directed transcription starts at the first nucleotide of $\mathrm{R}$ region with low efficiency (A). Binding of viral Tat transactivation protein to nascent transcript in TAR region (B) enhances polymerase activity via recruiting elongation factor composed of cyclin CycT1 and kinase CDK9 (C). CDK9 phosphorylates RNAP II, which result in dramatic stimulation of its processivity (D). Acetylation of Tat by P-CAF liberates it from TAR (E) and facilitates RNAP II-P-CAF association to further stimulate transcription elongation $(\mathbf{F})$.

Abbreviations: U3, 3' unique element; R, repeat element; RNAP II, RNA polymerase II; TAR, transactivation response element.

per se rather than association with specific chromatin ligands is central to the LEDGF/p75 mechanism (Meehan et al., 2009). It has been shown that binding to either DNA or protein molecules in chromatin as well as docking either within or outside the nucleosome is sufficient for the activity of LEDGF/p75 as an HIV-1 cofactor. According to these observations, while the basic LEDGF/p75 role is fulfilled without NDE, NDE domains might play more subtle roles in viral replication and selection of integration sites.

The importance of this protein for Lentivirus infectivity is not limited to the functions presented above and can be expanded by the following activities: enhancement of the nuclear import of PIC, stimulation of IN strand transfer and protection of IN protein from proteasomal degradation (for review see: Poeschla, 2008).

HIV-1 PICs also interact with other cellular proteins important for performing the PICs functions: integrase interactor 1 (INI-1/hSNF5), the first identified IN cofactor, one of the core subunits of the ATP-dependent chromatin remodeling complex SWI/ SNF that regulates expression of eukaryotic genes by modifying DNA/histone interactions (Kalpana et al., 1994); high mobility group protein A1 (HMGA1, formerly HMG I/Y), a non-histone chromosomal factor involved in transcriptional control and chromosomal architecture (Farnet \& Bushman, 1997; Miller et al., 1997; Hindmarsh et al., 1999); Ku70/Ku80 heterodimer, a component of the non-homologous DNA end joining (NHEJ) pathway engaged in double-stranded gap repair (Li et al., 2001); human heat shock pro- tein 60 (hHSP60), a protein chaperone (Parissi et al., 2001); barrier-to-autointegration factor (BAF), a protein highly conserved in metazoa with structural roles linking chromatin organization, nuclear architecture and gene regulation (Lin \& Engelman, 2003; Mansharamani et al., 2003); and TNPO3/transportin-SR2 (TRN-SR2), a nuclear import receptor for serine/arginine-rich (SR) proteins, important splicing regulators (Christ et al., 2008; Brass et al., 2008).

Gene expression

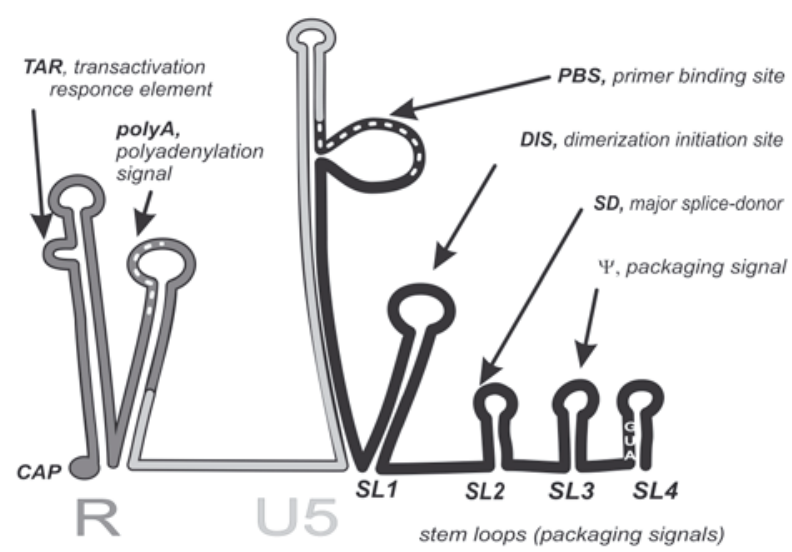

Figure 6. Schematic representation of RNA structure of HIV-1 5' UTR and 5' end of gag gene.

The $5^{\prime}$ end of viral mRNA is capped. The gag AUG start codon is highlighted. Dark-grey line shows R region, lightgrey line - U5 region. Dotted white lines depict positions of polyA and PBS sites on stem loops. RNA elements important for HIV-1 life cycle, including stem loops (SL) 1-4, are indicated. 
The late phase of the lentiviral life cycle starts when proviral DNA is transcribed by cellular RNA polymerase II (RNAP II) (Fig. 2F). HIV exploits the cellular transcriptional machinery in a sophisticated way. First, before the integration step is completed, the infected cell is arrested in the G2 phase of the cell cycle. This favors viral replication because the promoter elements present in the LTR seem to be most active in G2 (Goh et al., 1998). The block in the cell cycle progression is mediated by $\mathrm{Vpr}$ via indirect inactivation of the Cdc2 (CDK1)/cyclin B (CycB) kinase and $\mathrm{Cdc} 25$ phosphatase. These are regulators of the G2/M transition, possibly activated by the ataxia telangiectasia-mutated and Rad3-related (ATR) DNA damage response pathway (reviewed by: Amini et al., 2004; Le Rouzic \& Benichou, 2005; Andersen et al., 2008). However, there is also evidence that in the case of other lentiviruses (i.e., African green monkey SIV, SIVagm) the transactivation of LTR by Vpr is, in part, G2 arrest-independent (Zhu et al., 2001a).

Second, for initiation of its transcription, HIV usurps distinct cellular signaling pathways by using nuclear factor kappa-B (NF- $\mathrm{B})$ and nuclear factor of activated T cells (NFAT) (reviewed by: Cullen, 1991; Pessler \& Cron, 2004). After $\mathrm{T}$ cell activation by TCR, downstream from phospholipase C (PLC), NF$\kappa \mathrm{B}$ is activated via the diacylglycerol $/ \mathrm{Ca}^{2+} /$ protein $\mathrm{ki}$ nase $\mathrm{C}(\mathrm{PKC})$ pathway, whereas NFAT is activated by the IP3/ $\mathrm{Ca}^{2+} /$ calcineurin pathway (for review see: Tan \& Parker, 2003). NF-кB and NFAT binding sites along with binding sites for other cell-specific and constitutively expressed cellular transcription factors are located in the U3 enhancer/promoter region of $5^{\prime}$ LTR, upstream from the TATA box (Fig. 5).

The Tat protein is the main Lentivirus-encoded transcription transactivator. Tat interacts with the cis elements of the virus located in the junction between the $\mathrm{U} 3$ and $\mathrm{R}$ regions of the proviral $5^{\prime}$ LTR, to increase the processivity of RNAP II. Unlike typical transcriptional transactivators, Tat binds not to DNA, but to an RNA bulge of a stem-loop structure present at the $5^{\prime}$ end of all HIV-1 transcripts, known as the transactivation response element (TAR) (Fig. 6) (Feng \& Holland, 1988). Thus, Tat requires the initial transcription of TAR before it can stimulate further HIV transcription (for review see: Cullen, 1991; Brady \& Kashanchi, 2005; Barboric \& Peterlin, 2005). The basal transcriptional activity from the HIV LTR is very low and RNA synthesis is greatly increased (by more than two logs) when Tat is present (Fisher et al., 1986; Kao et al., 1987). Tat binds positive transcription elongation factor (P$\mathrm{TEFb}$ ), an elongation factor composed of cyclin T1 (CycT1) and kinase CDK9, and recruits it to TAR (Zhu et al., 1997; Wei et al., 1998). Subsequently, CDK9 phosphorylates the carboxy-terminal domain (CTD) of RNAP II, which in turn results in dra- matic stimulation of transcriptional processivity. The strength of the Tat-P-TEFb association is adjusted by autophosporylation of CDK9-stronger association (Garber et al., 2000) and acetylation of Tat-weaker association (Kiernan et al., 1999). This acetylation is mediated by p300/CREB-binding protein (CBP)-associated factor (P-CAF). Free acetylated Tat protein is now able to recruit P-CAF to phosphorylated RNAP II to further stimulate transcript elongation (Fig. 5). Acetylation of Tat is used to modulate the strength of the viral-host RNA-protein complex, thereby finetuning the efficiency of transcription elongation. It has recently been reported that in contrast to HIV-1 Tat, the bovine Lentivirus transactivator has evolved a high-affinity TAR interaction that does not require P-CAF-mediated acetylation. Additionally, HIV-2 Tat exploits an intermediate mechanism that uses a duplicated TAR element and CycT1 to enhance RNA binding (D'Orso \& Frankel, 2009).

Other results link the Tat-TAR axis to the latency, which is typical for Lentivirus infection. In HIV-1-infected resting CD4+ T cells, accumulation of Tat variants with impaired transactivation activity was observed, suggesting that impaired Tat activity may contribute to mechanisms responsible for latent infection with HIV (Yukl et al., 2009). In experiments involving Jurkat $\mathrm{T}$ cells infected with the HIV vector expressing Tat in cis, gene expression was progressively silenced as a result of the formation of restrictive chromatin structures at the viral LTRs (Pearson et al., 2008). This selectively impaired HIV transcription initiation and consequently decreased Tat production below the levels that are required to sustain HIV gene expression. This epigenetic mechanism of HIV transcriptional silencing might allow the virus to enter into latency.

Transcription of viral RNA begins at the first nucleotide of the $\mathrm{R}$ region in the $5^{\prime}$ LTR and polyadenylation occurs at the last nucleotide of $\mathrm{R}$ in the $3^{\prime}$ LTR. HIV-1 uses the cellular splicing machinery to express its genes (Fig. 2F). Cellular factors, in concert with both positive and negative cis elements within the viral genome, act to promote or repress splicing (Jablonski \& Caputi, 2009; reviewed by Stoltzfus \& Madsen, 2006). These cis elements, besides several splice donors (SD) and acceptors (SA), include: three exonic splicing enhancers (ESE), five exonic and intronic splicing silencers (ESS and ISS), and the guanosine-adenosine-rich exonic splicing enhancer (GAR ESE) that seems to be the key player in regulating the creation of both completely spliced and Revdependent vpulenv transcripts (Asang et al., 2008). Among the cellular factors, members of the SR family of phosphoproteins bind the enhancer elements and promote the use of splice sites, while members of the heterogeneous nuclear ribonucleoprotein (hnRNP) family of splicing factors bind the silencer el- 
ements and inhibit splice site utilization. The levels of these two classes of cellular splicing factors are regulated during HIV infection thereby affecting virus replication. Changes in expression and, possibly, localization of some splicing factors that modulate viral alternative splicing correlate with Tat cellular levels and virus production in macrophages. Reduced Tat expression and subsequent decline in virus production may contribute to the ability of HIV1 to persist in the macrophage reservoir (Dowling et al., 2008).

In addition to full-length genomic mRNA (about $9.2 \mathrm{~kb}$ in length), coding for the Gag and Gag-pol polyproteins, a number of various subgenomic species of mRNAs have been described in HIV-1-infected cells, with several slightly different mRNAs coding for the same protein (Schwartz et al., 1990; 1991; Purcell \& Martin, 1993; Swanson \& Stoltzfus, 1998). Of these, two classes of differentially spliced mRNAs can be detected in the cytoplasm: early, fully spliced (about $1.8 \mathrm{~kb}$ ) and late, partially spliced transcripts (about $4 \mathrm{~kb}$ ). The former encode the viral regulatory proteins Tat and Rev, along with the auxiliary protein Nef; the latter, accessory proteins: Vif, Vpu, and Vpr as well as the envelope protein Env (Fig. 1B).

Under physiological conditions eukaryotic cells block the nuclear export of unspliced mRNAs (recently reviewed by: Rougemaille et al., 2008; Schmid \& Jensen, 2008). Since lentiviruses exploit a variety of differentially and incompletely spliced transcripts, including unspliced genomic RNA, for expression of the complete repertoire of their proteins, they have developed mechanisms to escape this tight export control. The key protein in this process is the regulator of expression of viral proteins, Rev. Rev contains both a nuclear localization signal (NLS) and a nuclear export signal (NES), hence it shuttles between the nucleus and the cytoplasm (Fig. 2G) (reviewed by Pollard \& Malim, 1998). Rev, produced from early transcripts, accumulates in the cell and binds to the RNA Rev response element (RRE), a large (roughly 250 nucleotides in size) highly structured RNA element that is located in the env gene and is present in all unspliced and partially spliced HIV-1 RNAs (Malim et al., 1989; Pond et al., 2009). RRE constitutes a scaffold for Rev multimerization and the nascent Rev-RRE complex, via NES leucine-rich domains, directly binds CRM1, a member of the karyopherin family of nucleocytoplasmic transporters (Fornerod et al., 1997; Yi et al., 2002). This association is stimulated by Ras-related nuclear protein (Ran)/GTP bound to CRM1. In turn, CRM1 interacts with components of the nuclear pore complex (NPC), nucleoporin CAN/Nup214, and this interaction is essential for CRM1-mediated nuclear RNA export (Fig. 2F). In this way HIV "hijacks" the cellular pathway that is used to export small RNAs such as U snRNAs and rRNAs, preribosomal subunits, as well as proteins (reviewed by Cullen, 2009).

The synthesis of viral proteins is regulated by an interplay between host factors and cis-acting elements present in viral transcripts (Fig. 1). For example, a weak Kozak sequence surrounding the HIV-1 vpu AUG start codon promotes translation of the downstream env gene from bicistronic vpulenv transcripts, a process referred to as leaky scanning (Schwartz et al., 1992). However, more recent study unexpectedly showed that the different 5 ' untranslated regions (UTRs) present in alternatively spliced HIV-1 vpu/env mRNA isoforms affected Vpu synthesis, but not the downstream Env synthesis. Therefore, HIV-1 Env may also use an internal ribosome entry sequence (IRES) or the ribosome shunt translation mechanism to maximize Env production (Anderson et al., 2007). Although utilization of IRES to express genetic information via a cap-independent mechanism in Retroviridae is controversial, the presence of putative internal entry sites has been reported for various lentiviruses (Ohlmann et al., 2000; Buck et al., 2001; Brasey et al., 2003; Herbreteau et al., 2005).

The sequences coding for the Gag and Pol proteins, translated from a full-length unspliced RNA, are in different reading frames. HIV-1 requires a single -1 frameshift to produce the Gagpol polyprotein. The frameshifting occurs at an approximate rate of one Gag-pol for every twenty Gag molecules synthesized (Jacks et al., 1988). The signal for programmed frameshifting is provided by viral sequences including an upstream slippery sequence. For HIV-1 this sequence is AAUUUUUU (Watts et al., 2009) or UUUUUUA (according to earlier models). Another sequence engaged in the frameshifting is an RNA stem structure located downstream of the frameshift site. Interaction of tRNA with the ribosome, including not only the $\mathrm{P}$ and $\mathrm{A}$ sites, but also the E site, was postulated to be involved in this process (reviewed by: Bolinger \& Boris-Lawrie, 2009; Giedroc \& Cornish, 2009).

In addition to the translational mechanisms described above, retroviruses also employ cap-dependent translation enhancers. Post-transcriptional control elements (PCEs), located in the 5' UTRs, have been found in many viruses, including HIV-1 (reviewed by Bolinger \& Boris-Lawrie, 2009). PCEs are stem-loop RNA structures that specifically interact with RNA helicase A (RHA) and facilitate polysome loading and efficient viral protein production. Experiments involving the HIV-1 provirus and the PCE-HIV gag reporter RNA indicated that RHA downregulation diminishes HIV-1 gag translation independently of the Rev/RRE interactions or of global protein or RNA synthesis. 
Also other cellular proteins can bind viral RNA and affect its translation. A direct interaction between TAR and TAR-RNA binding protein (TRBP) enhances synthesis of viral proteins (Dorin et al., 2003). Downregulation of TRBP with small interfering RNAs (siRNAs) decreases basal expression from HIV-1 LTR two-fold and Tat-transactivated level up to ten-fold. Taken together, siRNAs against TRBP decreased the expression of viral genes and inhibited viral production up to five-fold (Christensen et al., 2007).

By contrast, hnRNP E1 exerts an opposite effect on HIV-1 translation. hnRNP E1 binds an HIV-1 exonic splicing silencer present in the bipartite tat/rev exon (ESS3a), although this interaction is not associated with a significant changes in viral RNA splicing. Instead, elevated levels of hnRNP E1 decreased Gag (Pr55 and p24), Env (gp160/gp120), and Rev synthesis (Woolaway et al., 2007).

Furthermore, analysis of the RNA structure of the entire HIV-1 genome has led to a number of intriguing findings (Watts et al., 2009). The reported correlation between the organization of the RNA and sequences encoding inter-domain loops in HIV proteins suggests that the RNA structure modulates the speed of ribosome during elongation in order to promote proper protein folding. Thus, differences in RNA sequence accessibility represent additional elements to control the structure and function of HIV proteins.

\section{Assembly and budding}

Production of HIV-1 particles by infected cell comprises a series of coordinated events: Gag dimerization and multimerization, binding of Gag complexes to genomic viral RNA (gRNA), transport of the Gag-RNA complexes, Gag-pol, Gag and Env to the site of assembly, and subsequent release of immature virions which later undergo maturation mediated by viral protease to become fully infectious (Fig. 2H-K).

The Gag precursor protein, Pr55, is responsible to balance translation of the full-length primary transcript (pre-mRNA) and its encapsidation (Anderson \& Lever, 2006). It has been shown that the Gag polyprotein has a bimodal effect on translation that depends on its concentration: stimulation at low and inhibition at high concentration. This transactivation of translation is presumably mediated by the MA domain of Gag, whereas inhibition results from an interaction between the NC domain and its binding site, packaging/dimerization signal $(\psi)$, in the HIV-1 5' UTR (Fig. 6). The proposed model assumes that Gag promotes its own synthesis until total covering of the HIV-1 5' UTR with nascent Gag molecules blocks further translation and directs this RNA to encapsidation. Yet, translation is not prerequisite for unspliced HIV-1 RNA to be directed for packaging into virions and pre-mRNA can function interchangeably as mRNA and as virion precursor RNA (vpRNA) (Fig. 2G,H) (Butsch \& Boris-Lawrie, 2000).

In contrast, HIV-2 unspliced RNA is packaged predominantly co-translationally. This discrepancy in the mechanisms used by lentiviruses to ensure that only full-length RNA is packaged can be explained by the differences in the position of $\psi$ with respect to the major splice donor (SD). Unlike in HIV-1, the HIV-2 packaging/dimerization signal is located upstream from the 5' SD. According to this observation, both HIV-2 RNA species, spliced and unspliced, contain the packaging signal. Thus, the biding of nascent Gag polyprotein to its own template provides a signal for encapsidation of unspliced RNA exclusively (Kaye \& Lever, 1999). However, further studies have revealed that transacting encapsidation of HIV-2 vector RNA is possible in a situation when $\psi$ region-mutated RNA is unable to efficiently capture newly made Gag. The excess of Gag protein is therefore available in trans to other RNAs, including spliced species of RNA, that contain an intact packaging signal. This and other results suggest that HIV-1 is able to produce Gag in larger quantities than HIV-2 and it can bind to RNAs anywhere in the cytosol, whereas putative HIV-2 Gag signals might direct it to specific subcellular compartments (Griffin et al., 2001).

While all secondary structures in HIV-1 5' UTR together with the major packaging signal are important for efficient RNA encapsidation, stem loop 1 (SL1) contains dimerization initiation site, DIS (or dimer-linkage structure, DLS) (Fig. 6) (reviewed by Russell et al., 2004). This raises the question if dimerization of RNA is a prerequisite for its packaging. If this is the case, the dimerization could function as a molecular switch that negatively regulates translation and promotes encapsidation. However, DIS is located upstream of SD (SL2) and thus is present in all spliced RNAs as well. On the other hand, the dimerization signal extends into the $5^{\prime}$ portion of gag gene (SL4), and some of its elements lie downstream of the major splice donor. This would provide a convenient mechanism by which only unspliced RNA would be able to dimerize and subsequently become encapsidated. Generally, the encapsidation and dimerization signals overlap in viral genomic leaders (Fig. 6) and the mechanism linking dimerization and packaging of HIV gRNA is still poorly understood. Nonetheless, the packaging of the genome requires specific recognition of dimeric, full-length viral RNA transcript by the NC domain of Gag (Fig. 2H).

An example of the MLV gRNA dimerization mechanism provides valuable insight into this problem. It was observed that MLV RNA dimerization 
triggers a conformational change within the packaging signal exposing elements that bind NC with high affinity and thus promote packaging of the diploid genome (D'Souza \& Summers, 2004). Consistently with this observation, it has recently been shown that although HIV virion packages two RNA molecules, it takes time to form fully matured dimers and this process requires viral protein maturation (Song et al., 2007). The authors propose a model in which the Gag and Gag-pol polyproteins assemble into a nascent virion containing two gRNA monomers or a fragile dimer. This requires the NC domain of Gag, which may recognize the fragile dimers better than monomers. At this step, DIS is unexposed. Following proteolytic maturation of the viral polyproteins, which is initiated only after the virion buds, products of this maturation (mainly the p9 intermediate and mature p7 NC) switch the DIS from a buried to an exposed conformation. During the first $30 \mathrm{~min}$ after virion release, all monomers (fragile dimers) become DIS-DIS "kissing" dimers. After the following several hours, the increasing number of released NC molecules stabilizes full RNA-RNA contacts. Additionally, not only the protease activity and NC maturation appear to be crucial for gRNA dimerization (Kafaie et al., 2008), but also the ratio of the Gag/ Gag-pol proteins in the nascent virion as well as CA mutations can impair this process (Shehu-Xhilaga et al., 2001; Kafaie et al., 2009).

Viral structural proteins and the newly synthesized viral RNA molecules migrate to the site of HIV-1 assembly stabilized by both the actin and tubulin cytoskeleton (Fig. 2H) (Jolly et al., 2007). The Gag precursor protein Pr55 plays a central role in virus assembly and is sufficient for production of noninfectious virus particles in the absence of other viral proteins (Gheysen et al., 1989; reviewed by Resh, 2005). Unlike other enveloped viruses, HIV leaves the cell via one of two budding pathways depending on the cell type from which it is exiting. HIV released from $\mathrm{T}$ lymphocytes predominantly buds through the cell membrane and acquires its envelope components from the plasma membrane, whereas virus replicating in macrophages usually exits the cell via vesicles of the endosomal network (reviewed by: Kolegraff et al., 2006; Bukrinskaya, 2007).

In T cells, HIV-1 is believed to assemble at and bud from so-called lipid rafts (Nguyen \& Hildreth, 2000), plasma membrane (PM) microdomains that are enriched in cholesterol and sphingolipids and serve as platforms for protein-lipid interactions and for the assembly and/or budding of a range of enveloped viruses (reviewed by Waheed \& Freed, 2009). The MA domain of Gag is responsible for its targeting and association with PM (Saad et al., 2006). Membrane binding is mediated by insertion of the myristoyl group of MA into the lipid bilayer and by a patch of basic residues which binds acidic phospholipids, particularly phosphatidylinositol 4,5-bisphosphate $\left(\mathrm{PIP}_{2}\right)$. The interaction with lipid rafts promotes Gag oligomerization (Ono et al., 2007) which, however, does not directly involve the MA domain. Instead, critical contacts are made by the C-terminal domain of CA, the adjacent SP1 spacer, the NC region, and, to some extent, the N-terminal domain of CA (reviewed by Ganser-Pornillos et al., 2008). During virion assembly, the MA domain of Gag associated with the PM also facilitates envelope protein binding to the site of budding via an interaction with the transmembrane (gp41) subunit of Env. Mutations in MA that abrogate viral envelope incorporation into virions also prevent Env association with PM (Bhattacharya et al., 2006; Bhatia et al., 2009). The Gag-pol precursor, Pr160, cannot form virus particles on its own and is directed into the assembling particle also through its interaction with Gag (Smith et al., 1993; Srinivasakumar et al., 1995). The C-terminal fragment of the Gag domain in the Gag-pol polyprotein is proposed to be responsible for this interaction. Particularly, deletions in the region immediately C-terminal to the major homology region (MHR) of the HIV-1 CA in Gag-pol markedly affect both virus assembly and the incorporation of Gag-pol (Chien et al., 2006).

Plasma membrane is the primary site of productive HIV-1 assembly in T lymphocytes as well as in most transformed cell lines (Finzi et al., 2007b). Nonetheless, it has recently been shown that under some circumstances efficient virus production can occur intracellularly (Joshi et al., 2009). In the case of macrophages, HIV-1 buds predominantly into endosomes or structures resembling multivesicular bodies (MVB) and is presumed to be released from these cells via exocytosis (Nguyen et al., 2003; Kramer et al., 2005). Since there is also a biological pathway that is used by a number of cell types, for example hematopoietic cells, to secrete microvesicles into the extracellular space, HIV might "hijack" that physiological pathway for leaving the infected cell (Gould et al., 2003). Although the exact nature of the signals that direct proteins necessary for virus assembly to either cell membrane or exosome is not known, it is clear that two distinct pathways for HIV-1 Gag targeting exist, one to the lipid rafts of the PM and the other to the MVB (Ono \& Freed, 2004).

Lentiviruses, as many other enveloped viruses, complete their replication cycle by budding through the cellular membrane (Fig. 2I). Since the virion envelope is derived from the host cell membranes, virus egress presents a problem because dissociation from the host membrane is not an easy or spontaneous step. In contrast to virus cell entry, when gp41 conformational changes provide free energy sufficient to promote membranes fusion (Fig. 2B) (see 
section on Entry), no such viral protein is known to exist to overcome the energy barrier required for the membranes' separation. Thus, viruses have evolved mechanisms that usurp the physiological pathway used by cells to create cytoplasm-containing vesicles within endosomes. The observation that mutations in the p6 domain of Gag prevent the release of budded virus particles from the cell surface has shed light on this issue (Göttlinger et al., 1991). Analysis of the p6 region revealed a PTAP amino acid motif, mutation of which was responsible for this defect (Göttlinger et al., 1991; Huang et al., 1995; Demirov et al., 2002). This sequence interacts with cellular components of the endosomal sorting complex required for transport (ESCRT) pathway to mediate virus release (reviewed by: Bieniasz, 2006; Chen \& Lamb, 2008; Carlton \& Martin-Serrano, 2009).

An alternative and very efficient way for virus spreading is cell-to-cell transmission (Sato et al., 1992). This transmission takes place at intercellular contact sites, so-called virological synapses (VS) that resemble immunological synapses. In the case of HIV-1, the VS is created by the recruitment of CD4 receptor, CXCR4 and CCR5 coreceptors, and lymphocyte function-associated antigen 1 (LFA-1) from the uninfected (target) cell and viral Env, Gag and adhesion molecules (intercellular adhesion molecule, ICAM-1) in polarized, lipid raft-like patches on the effector (donor) cell (Jolly et al., 2004; 2007; Jolly \& Sattentau, 2005; Wang et al., 2009b). However, it has been demonstrated recently that HIV transmission between $\mathrm{T}$ cells occurs efficiently in the absence of adhesion molecules (lack of LFA-1-ICAM-1 interactions) and that the Env - CD4 interactions are the main driving force of the VS creation between infected and uninfected CD4+ $\mathrm{T}$ cells (Puigdomènech et al., 2008). Interestingly, apart from the VS formation, HIV can be transmitted between T lymphocytes over relatively long distances using nanotubular connections, typical of these cells (Sowinski et al., 2008).

Initially, virus particles are released from the infected cell in an immature form containing a spherical shell of structural proteins underneath the virus membrane (Gag-Gag lattice with interactions mediated primarily by the CA and SP1 domains), but not a central cone-like core (Fig. 2J). During or shortly after virus budding from the plasma membrane, viral protease cleaves the Gag and Gag-pol polyprotein precursors to generate the mature Gag and Pol proteins. This processing takes place as an ordered cascade of cleavage reactions (the last one liberates SP1 from $\mathrm{CA}$ ), leading to the formation of the inner core (the NC/RNA complex condenses at the center), development of a conical-shaped core shell (CA lattice), and conversion of the immature virus particle into an infectious virion (Fig. 2A) (reviewed by: Bukrinskaya, 2007; Ganser-Pornillos et al., 2008).
The mature, infectious virus is now able to initiate another round of infection in a new target cell.

\section{CONSTRUCTION OF SAFE AND EFFICIENT HIV-1-BASED LENTIVIRAL VECTORS}

Because of their unique ability to transduce nondividing cells, Lentivirus-based vectors have great potential for delivering therapeutic genes to cells. However, because of safety concerns due to the potential for horizontal and cross-species transmission of recombined chimeric lentiviruses, the design of such vectors should include fundamental safety principles.

To address such safety issues several monitoring assays have been developed to test for the presence of replication competent lentiviruses (RCLs) in vector preparations, the possibility of vector mobilization from transduced cells, the persistence of vector-positive cells, and the abnormal clonal expansion of vector-modified cells (discussed by: Connolly, 2002; Manilla et al., 2005; Sastry \& Cornetta, 2009). Useful tips for vector construction and methods to test the quality and safety of vector lots can be found in Guideline on Development and Manufacture of Lentiviral Vectors published on-line in 2005 by EMEA's Committee for Medical Products for Human Use (CHMP) (see List of websites [9]). Some general criteria to be considered when conducting risk assessments for research involving lentiviral vectors are discussed in a similar document, Biosafety Considerations for Research with Lentiviral Vectors, published in 2006 by the U.S. National Institutes of Health (NIH)'s Recombinant DNA Advisory Committee (RAC) (see List of websites [10]).

Generally, to minimize risk associated with manufacturing and use of lentiviral vectors, all nonessential genes coding for accessory proteins and responsible for virulence, should be removed from the vector sequence. Additionally, the vector genome is usually split into several parts with limited sequence overlap in order to reduce to a minimum the possibility of recombination, vector mobilization and the generation of RCLs.

\section{EVOLUTION OF THE VECTOR DESIGN CONCEPT}

The first systems using replication-defective HIV-1 vectors were described in the early 1990s. Helseth et al. (1990) presented a trans-complementation assay for measuring the replicative potential of HIV-1 envelope glycoprotein mutants. The method utilized two plasmids: one containing an HIV-1 provirus with a deletion in the env gene and a chloramphenicol acetyltransferase (CAT) gene replacing the 
nef gene, and the rev and env either mutated or left intact under the control of the HIV-1 LTR. The idea to design a single-step replication assays with transient complementation of Env function was implemented to exclude the influence of proviral cis-acting mutations and multiple rounds of replication on the level of reporter gene expression.

In other initial experiments employing a Lentivirus as a gene delivery tool, HIV-1 env sequences were deleted and replaced by Escherichia coli gpt drug resistance gene driven by internal SV40 promoter (Page et al., 1990; Landau et al., 1991). The second plasmid provided either the gp120 glycoprotein or a heterologous Env protein. Although in this system the complete set of viral ORFs coding for regulatory and accessory proteins was present, HIV HXB2 molecular clone used for the plasmid construction was bearing attenuating mutations in $v p r, v p u$, and nef. Surprisingly, in the absence of Env, infected cells were able to produce non-infectious virions with the characteristics of gp120-containing particles, suggesting that Env is not necessary for virus particle for- mation. However, incorporation of envelope glycoproteins originating from different viruses allowed pseudotyped vectors to broaden their cell tropism.

In another early study the packaging component was separated into two different plasmids, one expressing the gag, pol, tat, and vif genes, and the other expressing env and rev (Poznansky et al., 1991). This system provided trans-acting viral functions that permitted the transfer of the HIV-1-derived vector without the generation of RCL. The two-plasmid packaging system was later extensively modified in experiments conducted to verify the roles of HIV-1 cis-acting elements in gene transfer into cells (Parolin et al., 1994). A plasmid expressing gag, pol, vif, and tat genes was constructed by replacement of the 5' HIV-1 LTR with the cytomegalovirus (CMV) immediate-early promoter (CMVie) and deletion of the $\psi$ sequence. The second plasmid expressed the rev and env genes under the control of HIV-1 LTR, but both plasmids contained heterologous polyA signals derived from SV40. The vector plasmids encoding a selectable marker neomycin phosphotransferase

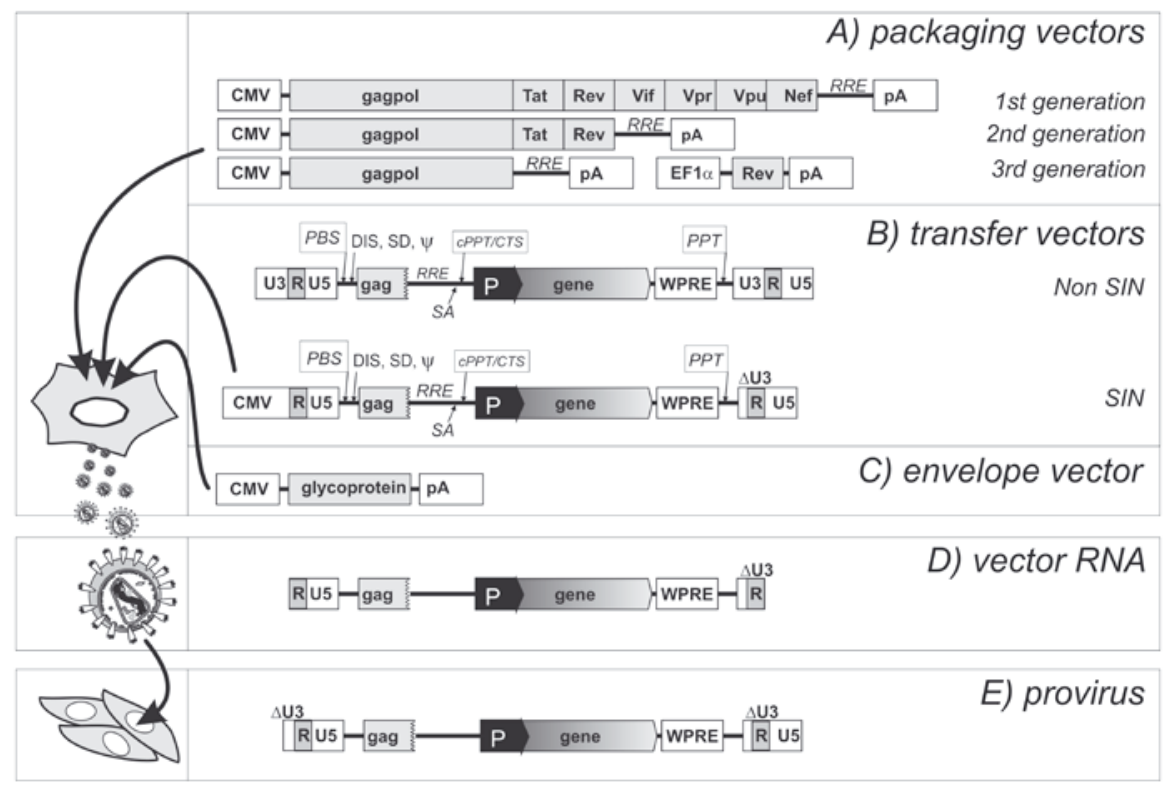

Figure 7. Lentivirus-based gene delivery system.

A-C. Plasmids used for transient transfection of producer cells. A. Packaging vector plasmids. First-generation vector contains all regulatory and accessory viral genes expressed from CMV promoter. Second-generation vector encodes only Tat and Rev proteins. Third-generation packaging system consists of two plasmids: one encoding Gag and Gag-pol polyproteins, the second - Rev protein. B. Transfer vector plasmids. In non-SIN vectors viral RNA is expressed from intact 5' LTR. SIN (self-inactivating) vector bears deletion in U3 region $(\Delta \mathrm{U} 3)$, which inactivates transcription of entire viral RNA after provirus integration. C. Envelope vector. Depending on glycoprotein used, different viral pseudotypes are formed. D. Schematic representation of vector genomic RNA. In presented setting, cotransfection of either second-generation packaging vector or third-generation vectors along with SIN transfer vector and envelope vector results in formation of viral particles that contain dimeric RNA bearing $\Delta \mathrm{U} 3$ mutation. E. After provirus integration into host cell DNA, transcription from mutated 5' LTR (duplicated 3' $\Delta \mathrm{U} 3$ ) is abrogated.

Abbreviations: CMV, cytomegalovirus immediate-early promoter; EF1 $\alpha$, human elongation factor 1- $\alpha$ promoter; gag, 5' portion of gag gene containing dimerization/packaging signals; PBS, primer binding site; DIS, dimerization signal; SD, splice donor site; SA, splice acceptor site; $\psi$, packaging signal; cPPT, central polypurine tract; CTS, central termination sequence; RRE, Rev response element; PPT, polypurine tract; pA, polyadenylation signal; $\Delta \mathrm{U} 3$, SIN deletion in U3 region of 3' LTR; P, internal promoter for transgene expression; WPRE, woodchuck hepatitis virus (WHV) post-transcriptional regulatory element. 
(neo), contained the $5^{\prime}$ LTRs, PBS, $\psi$ and $5^{\prime} \mathrm{SD}$, as well as $3^{\prime}$ genomic sequences, including PPT and LTR. Studies using these vectors revealed that the $5^{\prime}$ portion of the gag sequence inserted into the vector plasmid significantly enhanced marker gene transfer efficiency, possibly because a part of the HIV-1 packaging/dimerization signal is present in the extreme $5^{\prime}$ part of the Gag coding region (i.e., SL4; see section on Assembly and Budding and Fig. 6) (Clever et al., 1995). A positive effect was also observed upon inserting RRE sequences but only for those vectors which contained gag sequences (Parolin et al., 1994).

Other early HIV-1 vector systems involved the vesicular stomatitis virus G glycoprotein (VSVG) and displayed relatively high titers. Additionally, such particles were easy to concentrate by ultrafiltration or ultracentrifugation and were able to infect a range of targets, including nondividing cells, demonstrating their superiority over simple retrovirus-based systems (Reiser et al., 1996; Akkina et al., 1996).

The further development of lentiviral vector systems was based on the concept of separating the cis-acting sequences that are essential for vector RNA synthesis, packaging, reverse transcription and cDNA integration, from the trans elements that encode viral enzymes as well as structural and accessory proteins. Hence, such a system typically consists of: a packaging expression cassette(s) (helper), a vector cassette (transfer vector), and an envelope expression cassette (Fig. 7A-C). This concept was originally developed in the context of gamma-retroviral vectors.

\section{Packaging expression cassette(s) (helper plasmid)}

The packaging cassette expresses viral enzymes and structural proteins necessary for infectious particle formation, with the exception of Env. The original first-generation lentiviral vector system developed in 1996 contained the accessory proteins Vpu, Vpr, Vif, Nef, and the Rev, and Tat regulatory proteins as functional components of the packaging plasmid. Further refinements included eliminating the LTRs as well as $\psi$ and PBS sequences (Naldini et al., 1996a; 1996b). This prevented the packaging of full-length mRNA encoding trans elements into nascent vector particles. However, RRE and the 5' SD site remained unchanged, allowing normal mRNA processing and Rev-dependent export from the nucleus. In the absence of native LTRs, RNA synthesis was driven by promoters derived from other viruses, usually CMV or RSV (Rous sarcoma virus), whereas the polyA signal was adopted from SV40 or insulin gene (Fig. 7A).

These packaging constructs were subsequently refined by eliminating all accessory proteins that are associated with virulence and cytotoxicity (Ta- ble 1) and are not required for virus replication in vitro (Gibbs et al., 1994), eventually leading to the creation of the second-generation packaging cassette (Zufferey et al., 1997; Kafri et al., 1997; Kim et al., 1998; Mochizuki et al., 1998). In this system only Rev and Tat proteins were expressed together with the Gag and Gag-pol polyproteins (Fig. 7A).

In order to further improve the biosafety of the system, rev was later placed on a second plasmid, while tat was completely removed. The Tat function was replaced using modified 5' LTR enhancer/promoter elements containing strong, constitutive RSVor CMV-derived promoters in the corresponding vector constructs (Fig. 7A). This systems is referred to as a third-generation packaging cassette (Dull et al., 1998). In some ways it resembles the system described earlier by Parolin et al. (1994), but is much more streamlined. Fortunately, all those manipulations did not substantially affect vector production (titer) or infectivity. Moreover, the number of recombination events needed for the potential generation of an RCL increased considerably, and potential RCLs would not contain any proteins involved in wild-type virus virulence and pathogenicity.

However, homologous recombination was still possible since there were overlapping HIV sequences present in the helper and vector plasmids. These sequences encompass the RRE cis-element and a portion of the gag gene. RRE cannot be easily eliminated because it is necessary for efficient expression of HIV-1 gag and pol genes from the packaging construct. The reason for this requirement is low GC content and suboptimal codon usage in wild-type HIV mRNA. This causes instability of the RNA, which can be rescued from degradation by Rev binding. Codon optimization of the packaging cassette by changing the codon bias of the HIV-1 gag-pol to that of the human genome removed the homology with gag portion present in the transfer vector and led to efficient protein synthesis in the absence of Rev. This enhancement was shown to be mediated by increased mRNA stability and transport (Ngumbela et al., 2008). Thus, it allowed construction of packaging cassettes that do not contain an RRE (Kotsopoulou et al., 2000). It was also shown that production of the Gag-pol polyprotein from the codon-optimized mRNA is Rev-independent and that this RNA does not use the CRM-1-mediated nuclear export pathway. However, proper vector cassette expression remains Rev-dependent due to the requirement of a portion of gag for efficient packaging of the vector RNA (Parolin et al., 1994). A complete removal of Rev from the system, even if the helper plasmid lacked the RRE sequence, resulted in a significant (five-fold) reduction of vector titers when compared with the Rev/RRE containing systems (Kotsopoulou et al., 2000). 
Other successful attempt to establish a Revindependent packaging system was made by inserting the constitutive transport element (CTE) from Mason-Pfizer monkey virus (MPMV) into the HIV-1 sequence (Bray et al., 1994). Rev co-expression was found to be dispensable for transgene expression from CTE-containing vectors and titers obtained were roughly equivalent to the titers obtained with Rev-containing vectors (Srinivasakumar et al., 1997). The CTE function is similar to that of RRE (Rizvi et al., 1996). Moreover, if it stabilizes RNA to a grater extent than RRE does or enhances polysome loading of unspliced RNA (Swartz et al., 2007) it would be an additional benefit to put it in place of RRE. However, conflicting results with regard to the contribution of CTE itself have been reported (Kim et al., 1998). Nevertheless, as demonstrated more recently by Oh et al. (2007), an individual copy of the CTE is unable to replace the RRE in enhancing the production of functional vector particles and the incorporation of the multiple CTE sequences, in both the transfer or packaging plasmid, is required to eliminate the need for the expression of the rev gene during vector production.

Safety improvement in the design of vector packaging systems is still an area of intensive research. For example, the sequences encoding HIV enzymatic and structural proteins were split into two cassettes: one expressing the Gag/PR polyprotein and the other - the $\mathrm{Vpr} / \mathrm{RT} / \mathrm{IN}$ fusion protein. This prevented vector-mediated transfer of a functional gag-pol sequence into target cells (Wu et al., 2000; Kappes et al., 2003).

Attempts to create hybrid lentiviral vector systems exploiting the limited sequence homology between other members of the genus have also been pursued. It was shown that HIV-1 vectors can be effectively packaged by SIV core particles (White et al., 1999; Pandya et al., 2001). HIV-2 transfer vector RNAs can be encapsidated by the HIV-1 packaging machinery, or alternatively, HIV-1 vector can be packaged by a chimeric construct with the leadergag region of HIV-2 replaced with that of an HIV-1 packaging construct (Sachdeva et al., 2007).

Furthermore, lentiviral particles can be produced using transient expression of lentiviral packaging genes from a recombinant adenoviral vector. In this experimental setting cells stably transfected with a SIV-based vector cassette were transformed into lentiviral vector producer cells by infection with adenoviral "lenti-pack" vectors (Kuate et al., 2004). In a more recent study Semliki Forest virus (SFV) was used to produce recombinant pseudotyped HIV-1 virions. In the SFV/HIV hybrid system SFVderived packaging cassettes allowed encapsidation of SFV/HIV-1 vectors bearing both cis and trans HIV elements, and enabled transfected cells to produce transducing-competent chimeric SFV/HIV particles (Del Vecchio et al., 2009).

\section{Vector cassette (transfer vector)}

The vector cassette expresses full-length vector mRNA which contains all cis-acting elements necessary for efficient packaging, reverse transcription, nuclear import and integration into the host genomic DNA. Typically, the vector plasmid contains a transgene expression cassette with the gene of interest driven by an internal promoter, usually positioned between the $3^{\prime} \mathrm{Tat} / \operatorname{Rev} \mathrm{SA}$ site and the 3' LTR (Fig. 7B). The design of vector cassette has evolved in parallel with the development of packaging cassette. Early constructs contained intact $5^{\prime}$ and $3^{\prime}$ LTRs, and, thus, transcription was Tat-dependent. The resulting full-length transcript was comprised of TAR, PBS, SD and $\psi$ sequences present in the $5^{\prime}$ UTR, a 300-400 bp 5' portion derived from the gag gene (sometimes cloned out of frame or bearing nonsense mutation), RRE sequences, a splice acceptor (SA) site as well as PPT and polyA sequences within the $3^{\prime}$ LTR. The transgene with its own promoter was placed in between these sequences (Naldini et al., 1996a; 1996b; Poeschla et al., 1996).

Earlier reports showed that heterologous enhancer/promoters inserted into the HIV-1 LTR in place of the $\mathrm{T}$ cell-specific transcription factor (LEF1/TCF-1 $\alpha$ ), NFAT, NF- $\mathrm{BB}$, and Sp1 transcription factor binding sites in the U3 region (Fig. 5) drive transcription from the chimeric LTRs in the absence of Tat and that such modified viruses are replicationcompetent (Chang et al., 1993). Weak transactivation by Tat was still evident and the infectivity of the viruses was cell type-dependent. Based on these early studies, Tat-independent transcription of the vector cassette was accomplished by replacement of the enhancer/promoter sequences in the U3 region of $5^{\prime}$ LTR with a strong heterologous promoter derived from CMV (Fig. 7B) (Kim et al., 1998), as it had been done previously for the MLV (Soneoka et al., 1995). Such transfer vectors are now an integral part of the third-generation packaging systems lacking Tat. However, transcription from such chimeric LTRs in producer cells still could be upregulated by Tat, which resulted in higher titers and increased transduction efficiency of newly made vector particles by $40 \%$ (Dull et al., 1998). This could be explained by the fact that the TAR element is still present in nascent transcripts (Fig. 6).

Deletion of the enhancer/promoter sequences in the U3 region of $3^{\prime}$ LTR (120-400 bp; $\left.\Delta \mathrm{U} 3\right)$ has led to the creation of so-called self-inactivating (SIN) vectors (Fig. 7B) (Dull et al., 1998; Miyoshi et al., 1998; Zufferey et al., 1998; Iwakuma et al., 1999). This idea was first introduced more than a decade earlier 
in the context of MLV-derived retroviral vectors ( $\mathrm{Yu}$ et al., 1986). When viruses derived from such transfer constructs are used to infect cells, the deletion is reproduced in the 5' LTR during reverse transcription, resulting in the transcriptional inactivation of the provirus (Fig. 7D, E). The most important improvement of viral vector safety offered by the SIN design is the reduction in expression of full-length transcripts available for packaging, which minimizes the possibility of vector mobilization upon superinfection with wild-type HIV (Bukovsky et al., 1999). The chance of RCL formation is also reduced due to further elimination of sequences homologous to wild-type virus. Additionally, SIN deletion should make insertional mutagenesis less likely, preventing the transcriptional interference with promoter/ enhancer elements present in the host genome. Actually, in the recently developed cell culture assay for insertional mutagenesis, SIN deletion of the promoter/enhancer region from HIV-based vector LTRs resulted in a complete abrogation of the insertional gene activation, frequently observed for both lentiviral and gamma-retroviral vectors (Bokhoven et al., 2009).

Indeed, recent studies have identified transcriptionally active LTR as the major determinant of vector genotoxicity (Montini et al., 2009). In a mouse tumor-prone HSPCs transplantation model, a lentiviral vector with a chimeric onco-retroviral LTR increased tumor onset to a level expected for a simple retrovirus-based vectors. In a parallel experiment, both lentiviral and gamma-retroviral vectors with a SIN LTR appeared to be neutral. It was concluded that in addition to the aforementioned transcriptional interference mechanism, the placement of enhancer/ promoter sequences in the LTR upstream of a strong splice donor site may increase the probability of chimeric transcript formation and oncogene activation. Hence, for a safe vector design SD sites downstream of strong promoters should be avoided. In addition to the use of SIN-modified LTRs, moderately active internal promoters are recommended for vectors for clinical applications, especially when the weak $3^{\prime}$ LTR polyA signal serves to terminate transcription (see Heterologous PolyA Signals section below).

Integrated vector genomes containing fulllength 5' LTRs (Bukovsky et al., 1999; Evans \& Garcia, 2000; Levine et al., 2006) can be mobilized by infection with wild-type HIV virus. It has been shown that virtually all integrated proviral genomes are transcribed, albeit at low frequency dependent on the integration position. On the other hand, in the experiments employing $\mathrm{T}$ cells, only a small number of integrated HIV-1-based vectors expressed transgene from viral LTR. The vast majority of the vectors was silenced immediately after integration (Mok et al., 2007). Although SIN proviruses are much less likely to be mobilized, any transcript derived from a cryptic promoter present within the integrated sequences or in the surrounding host DNA, that includes the $\mathrm{R}$ region of the $5^{\prime}$ LTR, may give rise to an intact proviral DNA genome that can be reintegrated (Logan et al., 2004a; Hanawa et al., 2005). The mobilization of integrated SIN vectors during wildtype HIV superinfection has not been demonstrated yet, but careful design of a safe vector requires further efforts to resolve this potential problem (see section on Chromatin Insulators below).

Another possible source of viral genomes available for packaging are episomal forms of the vector DNA that circularizes and remains unintegrated in transduced cells. Such episomal forms of viral DNA, single- and double-LTR (1-LTR and 2LTR) circles, are diluted upon cell division (Van Maele et al., 2003), but in nonproliferating, quiescent cells they are stable and transcriptionally competent (Gillim-Ross et al., 2005) and, thus, can considerably contribute to the availability of full-length gRNAs for packaging and mobilization. However, in more recent study on HIV-1 preintegration transcription, the majority of 2-LTR circles appeared not to be active in directing RNA synthesis. It was concluded that the nonintegrated templates were mainly from the predominant DNA species, such as the fulllength, linear DNA (Iyer et al., 2009).

\section{Envelope expressing cassette}

The last element of a lentiviral vector system is an envelope cassette, which provides nascent particles with envelope glycoprotein (Fig. 7C). Since the genuine lentiviral envelope gene is removed from the system, an additional plasmid expressing a heterologous glycoprotein is used during vector production. Such an approach, called pseudotyping, offers several significant advantages: 1) increase in vector safety due to elimination of sequence homology with wild-type virus, 2) expanded or selective specificity of pseudotyped vector tropism towards target cells, 3) improvement of particle stability allowing virus concentration and long-term virus stock storage.

As was described above, Page et al. (1990) and Landau et al. (1991) were the first to demonstrate the ability of a recombinant Lentivirus-based vector to incorporate a heterologous envelope glycoproteins. They achieved relatively high titers $\left(10^{5}\right.$ colony forming units per $\mathrm{ml}, \mathrm{CFU} / \mathrm{ml}$, of preparation) and an altered cell tropism of the HIV-1 based vectors using eco- and amphotropic MLV Env or HTLV-I Env. Nevertheless, the glycoprotein of choice for the vast majority of vector systems currently in use is VSV-G. It offers unequaled titers $\left(10^{7}-10^{8}\right.$ transducing units per $\mathrm{ml}, \mathrm{TU} / \mathrm{ml}$, before concentration), excellent stability of the virions and 
allows virtually all mammalian (and insect) cell types to be infected. The first successful attempts to pseudotype lentiviral vectors with VSV-G were conducted concurrently by several groups (Naldini et al., 1996a; Reiser et al., 1996; Akkina et al., 1996; Poeschla et al., 1996). The VSV-G envelope facilitates vector entry via the endocytic pathway, which diminishes the requirement for viral accessory proteins for full infectivity (i.e., Nef; see section on Entry) (Aiken, 1997; Chazal et al., 2001). However, the mechanism responsible for cell binding as well as cellular receptors for this glycoprotein remain unknown. For example, an involvement of phosphatidylserine, an ubiquitous lipid of the cell membrane that was long believed to serve as a receptor for VSV-G, is currently controversial (Coil \& Miller, 2004; Carneiro et al., 2006).

One drawback of the production of pseudotyped vectors using VSV-G is that, due to its fusogenic properties ( $\mathrm{Li}$ et al., 1993), it is toxic to some mammalian cells (e.g., 293 cells) if constitutively expressed (Burns et al., 1993). Hence, the use of producer cell lines expressing packaging proteins for long-term virus production requires conditional production of this glycoprotein. Such retrovirus/Lentivirus producer cell lines have been constructed with tetracycline-regulatable promoters used to express VSV-G (Chen et al., 1996; Ory et al., 1996; Kafri et al., 1999; Klages et al., 2000; Farson et al., 2001; Ni et al., 2005; Cockrell et al., 2006; Broussau et al., 2008).

Another shortcoming for application of VSV-G-pseudotyped vectors in vivo is the complement- and antibody-mediated immune response directed against the envelope (DePolo et al., 2000; Higashikawa \& Chang, 2001). The complement-mediated inactivation can be alleviated by chemical modifications of the envelope glycoprotein. Successful extension of the vector half-life and an increase in transduction efficacy in vivo have been demonstrated with VSV-G conjugated with poly(ethylene glycol) (PEG) (Croyle et al., 2004).

\section{Targeting lentiviral vectors by pseudotyping}

The construction of optimal or tissue-specific envelopes is still one of the most important research objectives. For example, a chimeric RD114 glycoprotein, with the transmembrane and extracellular domains of the feline endogenous virus RD114 glycoprotein fused to the cytoplasmic domain derived from the amphotropic MLV 4070A Env, enables transduction of bone-marrow-derived mesenchymal stem cells (MSCs) with an efficiency

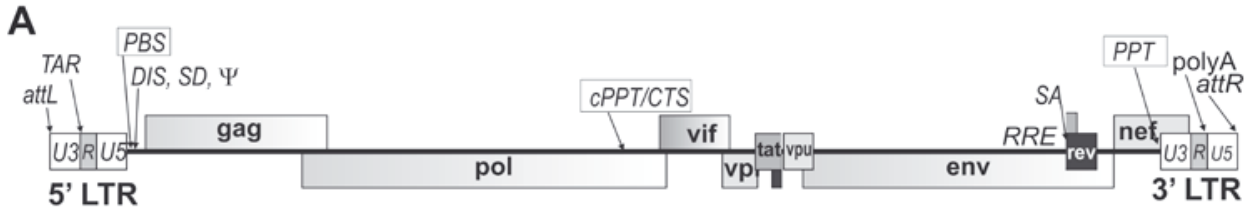

B

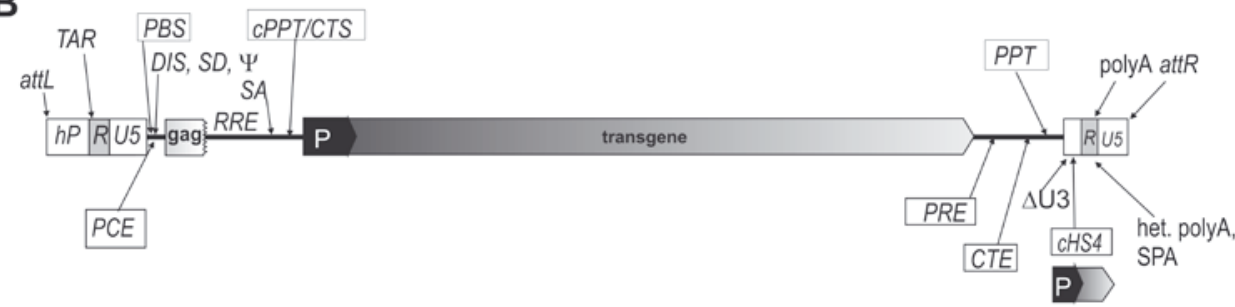

Figure 8. Comparison of wild-type HIV-1 provirus and optimized lentiviral vector.

A. Schematic representation of HIV-1 provirus. B. Typical cis-acting elements are marked above the vector. Below - examples of additional modifications introduced to Lentivirus-based vectors in order to elevate titer and/or transgene expression levels. After transfection, viral genomic RNA is synthesized using strong hybrid promoter placed at $5^{\prime}$ end of vector. Elimination of weak promoter/enhancer from 5' LTR enables Tat-independent transcription and use of third-generation packaging system. Incorporation of post-transcriptional control element improves translation efficiency. In SIN (self-inactivating) vectors deletion in U3 region of 3' LTR $(\Delta \mathrm{U} 3)$ is duplicated during reverse transcription and integration, which results in abrogation of viral RNA transcription. $\Delta \mathrm{U} 3$ site can be used for insertion of chromatin insulator or transgene cassette and, thus, these elements will be duplicated in provirus. To improve polyadenylation of SIN vector transcript, heterologous or synthetic polyadenylation signals can be inserted into R region of 3' LTR. Post-transcriptional regulatory elements and RNA transport elements can be incorporated into 3' end of transgene to reinforce transcript 3' end formation, RNA stability and nuclear export.

Abbreviations: hP, hybrid promoter; attL, attR, left and right attachment sites; TAR, transactivation response element; gag, 5' portion of gag gene containing dimerization/packaging signals; PBS, primer binding site; DIS, dimerization signal; $\mathrm{SD}$, splice donor site; SA, splice acceptor site; $\psi$, packaging signal; cPPT, central polypurine tract; CTS, central termination sequence; RRE, Rev response element; PPT, polypurine tract; polyA, polyadenylation signal; $\triangle \mathrm{U} 3, \mathrm{SIN}$ deletion in U3 region of 3' LTR; P, internal promoter for transgene expression; PCE, post-transcriptional control element; PRE - posttranscriptional regulatory element; CTE, constitutive transport element; cHS4, chicken $\beta$-globin insulator; het. polyA, heterologous polyA signal; SPA, synthetic polyA signal. 
similar to that obtained with VSV-G pseudotypes, but with lower cytotoxicity (Zhang et al., 2004a).

In another study, lentiviral vectors pseudotyped with a glycoprotein derived from the Rabies virus PV strain exhibited the best performance and neuronal tropism among the tested envelopes. Rabies PV virus-pseudotyped vectors transduced neurons and other cells of neuronal origin with unequaled efficiencies when compared to other Lyssavirus glycoproteins, including VSV-G (Federici et al., 2009). Furthermore, the Rabies envelope glycoprotein facilitates in vivo retrograde transport of vector particles along neuronal axons, enabling remote gene delivery to the central nervous system (CNS) via peripheral virus injection (Mazarakis et al., 2001; Federici et al., 2009).

A serious disadvantage of the lentiviral vectors pseudotyped with VSV-G is their inability to infect cells blocked in the G0 phase of the cell cycle. However, a high level transduction of such relevant therapeutic targets as memory and naïve $\mathrm{T}$ cells was achieved by the incorporation into virions glycoproteins from Edmonston measles virus (MV). Presence of hemagglutinin, responsible for receptor recognition, and the fusion protein on the virus surface allowed the efficient transduction of resting cells via the MV receptors: SLAM and CD46 (Frecha et al., 2008a). Similarly, lentiviruses pseudotyped with the MV glycoproteins were able to infect quiescent B lymphocytes as well as leukemic cancer cells, B-CLL cells, blocked in the G0/G1 early phase of the cell cycle (Frecha et al., 2009). Another research group reported transduction of unstimulated primary B cells using retargetable MV envelope glycoproteins (Funke et al., 2008). In this study, hemagglutinin was engineered to display either the epidermal growth factor (EGF) or a single-chain antibody directed against CD20. Thus, cell infection was performed not through the MV receptor but via the EGF and CD20 receptors. Consequently, gene transfer to cells that expressed the targeted receptor was several orders of magnitude more efficient than to other cells.

In another recent report, a targeted lentiviral vector exploiting a natural ligand-receptor binding mechanism was used for modification of c-KIT (CD117) receptor-expressing cells in vitro and in vivo. To target CD117+ cells the vector surface was engineered to contain membrane-bound human stem cell factor (hSCF) (for specific receptor recognition) and a Sindbis virus glycoprotein-derived fusogenic molecule (FM) (for membranes fusion) (Froelich et al., 2009). The targeted vector was 3.6-5 times more effective in transducing CD117+ cells than control viruses. Similar strategy was used to target CD20-expressing B cells (Yang et al., 2006), cells expressing monospecific surface immunoglob- ulin recognizing CD20 ( $\alpha$ CD20) (Ziegler et al., 2008), dendritic cells expressing dendritic cell-surface protein DC-SIGN (Yang et al., 2008b), and CD3+ T cells (Yang et al., 2009). Notably, engineering (mutagenesis) of the fusion-active domain of the FM enabled enhancement of targeted transduction by a factor of $8-17$, possibly due to a wider $\mathrm{pH}$ range of activity observed in engineered FMs (Lei et al., 2009).

The strategies used for Lentivirus-based vector pseudotyping have been comprehensibly reviewed by: Verhoeyen \& Cosset (2004); Cronin et al. (2005); and Frecha et al. (2008b).

\section{ADDITIONAL ELEMENTS FOR VECTOR IMPROVEMENT}

Since HIV-1 virus can effectively package approx. $9.7 \mathrm{~kb}$ of its genome, reduction of the length of the viral vector backbone should increase its payload carrying capacity. In the work published by Cui et al. (1999), subsequent mutations and deletions of the viral regulatory elements, including SD, most of gag, and RRE, have brought down the content of the original HIV-1 sequences in the vector DNA to less than $550 \mathrm{bp}$. This should allow accommodation of more than $9 \mathrm{~kb}$ of foreign DNA. Yet the usefulness of such design was diminished by a decrease in vector titer, about half that of the wild-type construct. In another detailed study on lentiviral vector capacity, maximal vector size (including 1.6-2.2 kb of the virus backbone) has been estimated to approx. 13.5 kb (Kumar et al., 2001).

Thus, the design of a safe and efficient lentiviral vector requires both deletions of non-necessary sequences from the backbone and insertions of elements that are proven to have a positive effect on vector titer or transgene expression (Fig. 8).

\section{cPPT-CTS}

The role of CPPT and CTS in the HIV-1 life cycle was described in previous sections. Consistently with the cis-acting role of the central DNA Flap (about $170 \mathrm{bp}$ ) in reverse transcription and in the nuclear import of viral PIC, its re-insertion into HIV-1-derived gene transfer vectors strongly stimulates gene transfer efficiencies (up to ten-fold) in all cell types tested, both in vivo and ex vivo (Sirven et al., 2000; Park \& Kay, 2001; Zennou et al., 2001; Dardalhon et al., 2001; Baekelandt et al., 2002; Manganini et al., 2002; Nguyen et al., 2002; Van Maele et al., 2003; Breckpot et al., 2003; Giannini et al., 2003; Ao et al., 2004; Logan et al., 2004b). Interestingly, this positive effect of cPPT and CTS on the transduction efficiency depends neither on the position and number of DNA Flaps in the vector backbone nor on the Flap sequence (De Rijck et al., 2005; De Rijck \& Debyser, 2006). 
PRE and PCE

Another commonly used cis-acting element is the woodchuck hepatitis virus (WHV) post-transcriptional regulatory element (PRE), WPRE (Zufferey et al., 1999). Incorporation of this sequence (600 bp) in the $3^{\prime}$ untranslated region of a transcript increases overall transgene expression by more than six-fold (Oh et al., 2007). In the context of the FIV vector, a WPRE-containing construct gave a functional titer about ten times higher than the WPRE-lacking one (Pistello et al., 2007). Interestingly, in this experimental setup insertion of cPPT had no noticeable effect on the vector performance.

One should note that the WPRE element contains an ORF encoding a truncated peptide of the WHV X protein (WHX), which might be associated with hepatocellular carcinomas. However, neither expression of this peptide from a lentiviral vector nor its direct involvement in oncogenesis have been demonstrated. Besides, the potentially harmful sequences can be successfully eliminated from WPRE without negative effects on its functionality, which enables designing safe and efficient vectors for in vivo applications (Schambach et al., 2006; ZantaBoussif et al., 2009).

The exact role of WPRE in vector performance is unclear. One of its functions may be the reduction of viral readthrough transcription and, thus, improvement of transcript termination leading to higher titers and transgene expression (Higashimoto et al., 2007). Indeed, in a situation when the SIN design negatively affected promoter performance (see section Heterologous PolyA Signals below) insertion of WPRE near the 3' LTR reversed this effect (Salmon et al., 2000a). Post-transcriptional regulatory elements may additionally enhance intronless transgene expression through stabilization of the $3^{\prime}$ end of the transcript and improvement of the nucleocytoplasmic export of unspliced mRNAs. Also other than WPRE cis-acting elements (e.g., hepatitis B virus (HBV) PRE, HPRE) can be considered useful in in vivo applications (Sun et al., 2009).

Another cis-acting post-transcriptional element that may positively affect transgene expression is PCE (see section on Gene Expression). When the CMVie enhancer and spleen necrosis virus (SNV) PCE were inserted into a lentiviral vector backbone, they acted together to achieve coordinate increases in RNA synthesis and translation (Yilmaz et al., 2006). It was demonstrated that CMVie and PCE function synergistically to increase protein yield in transduced cells four-fold compared to a control vector lacking these sequences.

Heterologous polyA signals

Due to deletions in the U3 region containing polyadenylation "enhancers" (DeZazzo et al., 1992),
SIN vectors suffer from leaky transcription termination increasing the probability of readthrough into cellular genes, potentially oncogenes. Hence, incorporation of strong polyA signals is of great importance for safe vector design. An attempt to solve this problem was made by Schambach et al. (2007). Insertion of a $100 \mathrm{bp}$ sequence, comprising a recombinant direct repeat of the upstream polyadenylation enhancer element (or upstream sequence element, USE) derived from SV40, improved both the titer and gene expression from a viral vector. Additionally, this element suppressed readthrough more efficiently than WPRE did and was able to substitute for the WPRE functions to some extent.

Furthermore, replacement of the original polyA signal in the R/U5 regions of $3^{\prime}$ LTR with a bovine growth hormone polyadenylation (bGHpA) sequence significantly elevated the efficiency of SIN vectors (Iwakuma et al., 1999). The bGHpA signal was also successfully used to enhance expression of the second gene in vectors carrying multiple genes (Osti et al., 2006). However, insertion of a polyA signal between two LTRs in a lentiviral vector very often leads to a premature termination of the genomic transcript and greatly decreases the viral titer. Thus, the aforementioned experiments were conducted transient, only in cells transfected with the transfer vector plasmid. Indeed, the internal polyadenylation signals in lentiviral vectors reduce the viral titers. This undesired effect is promoter-dependent and can be avoided by using selected promoters (Hager et al., 2008). A similar positive effect on downstream gene expression was achieved when a synthetic polyadenylation (SPA) termination sequences were used and, to avoid disruption of viral mRNA production by the polyadenylation sequences in the middle of the vector, the expression cassettes were cloned in the antisense orientation relative to the viral LTRs (Tian \& Andreadis, 2009).

\section{Chromatin insulators}

Previously, Logan et al. (2004a) and Hanawa et al. (2005) reported the potential of transcription of integrated SIN vectors from cryptic promoters, either within or upstream of the vector genome. Worse still, particles containing such mobilized vector genome were capable of transferring the intact proviral DNA into target cells. In order to minimize such an undesirable phenomenon, a $1.2 \mathrm{~kb}$ fragment of the insulator element from the locus control region of the chicken $\beta$-globin gene, chicken hypersensitive site 4 (cHS4) sequence (Chung et al., 1993), was inserted into the vector LTRs. This resulted in a significant (to one-fourth of the control level) reduction in the full-length vector transcription (Hanawa et al., 2005).

On the other hand, insertion of insulator sequences into vectors can help maintain long-term 
transgene expression by suppression of chromosomal position effects (transgene silencing) resulting from integration into the host chromatin (Ramezani et al., 2003; Hino et al., 2004; Arumugam et al., 2007). The insulator sequence was also successfully used to facilitate expression of the second gene in vectors carrying multiple genes (Osti et al., 2006; Tian \& Andreadis, 2009), to diminish clonal dominance in cultures of human $\mathrm{T}$ cells containing integrated vector genomes (Evans-Galea et al., 2007), and to reduce basal expression from a regulatable promoter by shielding the vector from the effects of enhancer elements present in the neighboring host DNA (Pluta et al., 2005; Vieyra \& Goodell, 2007).

Fortunately for vector design, the size of the cHS4 insulator can be reduced to $400 \mathrm{bp}$ without impairing its barrier function (Aker et al., 2007), or even to $77 \mathrm{bp}$ if combined with a homologous region from the human $\mathrm{T}$ cell receptor alpha/delta BEAD-1 insulator (Ramezani et al., 2008). This is important, as lower viral titers were sometimes reported when doubled copies of full-length cHS4 were used to flank the expression cassette or when this large insulator fragment was cloned into 3' LTR (Hanawa et al., 2009; Nielsen et al., 2009; Urbinati et al., 2009). Observations suggest that this effect is caused by impaired RNA processing during transduction of the target cell, probably on the level of reverse transcription and integration. Hanawa et al. (2009) reported incorporation of the $250 \mathrm{bp}$-long core element of the cHS4 insulator. Use of this element rescued vector titer compared to the titer of $1.2 \mathrm{~kb}$ insulator-bearing virus. Additionally, the presence of this short core element significantly increased transgene expression from an internal promoter due to improved transcriptional termination and reduced the variability of expression caused by the position effects. However, results presented by other researchers showed that the core alone did not insulate viral vectors effectively and only combination of the core and distal $400 \mathrm{bp}$ of cHS4 sequences restored full insulator activity (Arumugam et al., 2009).

In summary, the evolution of the packaging cassette design and the optimization of the transfer vector have eventually led to the removal from lentiviral vector system of at least five proteins associated with HIV virulence, Tat, Nef, Vif, Vpr, and Vpu. This engineering coupled with pseudotyping makes the vectors based on lentiviral genome clearly distinct from the HIV virus in terms of their biology and, in consequence, ensures their safety as a gene delivery system. Furthermore, additional elements that are commonly incorporated into the vector backbones (e.g., cPPT and CTS, WPRE, PCE, heterologous polyA signals, and chromatin insulators) facilitate production of high titer virus stocks and elevate transgene expression levels.

\section{NONINTEGRATING LENTIVIRAL VECTORS}

The problem of a presence of the episomal, circularized forms of the vector DNA in infected cells was discussed previously in a context of the potential sources of viral genomes available for undesired mobilization and packaging (Vector Cassette (Transfer Vector) section). Nonetheless, recognition of the unique features of these LTR-containing circles has recently led to the development of so-called integration-deficient lentiviral vectors (IDLVs) or nonintegrating lentiviral vectors (NILVs) (for relevant review see: Sarkis et al., 2008; Wanisch \& Yáñez-Muñoz, 2009). In brief, the IDLVs resemble integrating vectors described in previous sections except that they have mutated integrase. Thanks to their episomal nature (integration frequencies are 3-4 logs below those of their integrating counterparts), the IDLVs have a greatly reduced risk of promoting insertional mutagenesis. Moreover, since active IN is required for the viral replication nonintegrating vectors should not support the HIV1-based replication if an unlikely recombination event generated an RCL. In the vectors designated for use in gene therapy additional mutations at the att sites would reduce integration frequencies of the vector mobilized by a wild-type virus.

The HIV-1-based vectors packaged with a mutated integrase maintain transgene expression in vitro and in vivo. Because they are integration- and replication-deficient this expression is transient in dividing cells. In nondividing cells, however, gene transfer by the IDLVs is relatively stable (Philippe et al., 2006). An episomal character of the nonintegrating vectors has also consequences in the stability of transcription since the IDLVs may not be a subject to the epigenetic silencing. Unfortunately, transgene expression levels observed for nonintegrating lentiviral vectors were much lower compared to those of their integrating counterparts (Bayer et al., 2008). Another shortcoming of the IDLVs is their reduced transduction efficiency (Okada et al., 2009).

Regardless of these limitations, nonintegrating lentiviral SIN vectors can be packaged in producer cells with an efficiency comparable to that of regular vector RNA (Ma \& Kafri, 2004). Moreover, the Lentivirus-based episomal system for gene delivery proved its usefulness in the genetic correction of the disorder in animal models (Yáñez-Muñoz et al., 2006), effective vaccination (see references in section on Lentiviral vectors in clinical gene therapy applications), gene silencing mediated by RNA interference (RNAi) (Wanisch \& Yáñez-Muñoz, 2009), transgene delivery to the adult and fetal CNS (Rahim et al., 2009) and to muscle in vivo (Apolonia et al., 2007) as well as in the transposase-directed genomic integration of the minimal gene expression cassette (Vink et al., 2009; Staunstrup et al., 2009). 


\section{TARGETED INTEGRATION OF LENTIVIRAL VECTORS}

The combination of episomal lentiviral vectors with the transposon-mediated transgene integration constitutes another step toward the construction of a safer gene delivery tool. The IDLVs provide an efficient gene delivery vehicle for the system whereas transposase directs transgene integration away from the transcriptionally active loci, favored by the regular integrase-proficient lentiviral vectors. This fact along with the absence of viral LTRs in a host cell genome minimizes the risk of the insertional mutagenesis or a proto-oncogene activation (Vink et al., 2009; Staunstrup et al., 2009).

Nonetheless, for gene therapy purposes, including either the gene repair or knock-down, the site-directed integration is highly desirable. In spite of the fact that the cells with modified genes can be isolated or enriched in the transduced/transfected ex vivo culture, the frequencies of derivation of such corrected cells are very poor with traditional gene targeting methods. An interesting attempt to achieve targeted gene repair was reported by Cornu \& Cathomen (2007). The system, developed for the correction of the endogenous mutation in the marker gene directly in cellular genome, was based on the IDLVs-mediated delivery of the repair template and the I-SceI yeast meganuclease-directed homologous recombination. Expression of the nuclease from separate vector created a double-strand break within the target locus, which enabled recombination with the template DNA. The marker expression was rescued in up to $12 \%$ of the cells. Although these results are very promising, described above method requires the initial engineering of recognition sites for the I-SceI nuclease. Similar mechanism of the site-directed integration was also demonstrated with another non-viral enzyme, yeast Flp recombinase (Moldt et al., 2008).

The possibility to avoid host genome manipulations is offered by the application of so-called designer nucleases that are able to cut target DNA at specific genomic sequences. Recent attention focuses on the chimeric zinc-finger nucleases (ZFNs) that can be engineered to target desired sites. Lombardo et al.

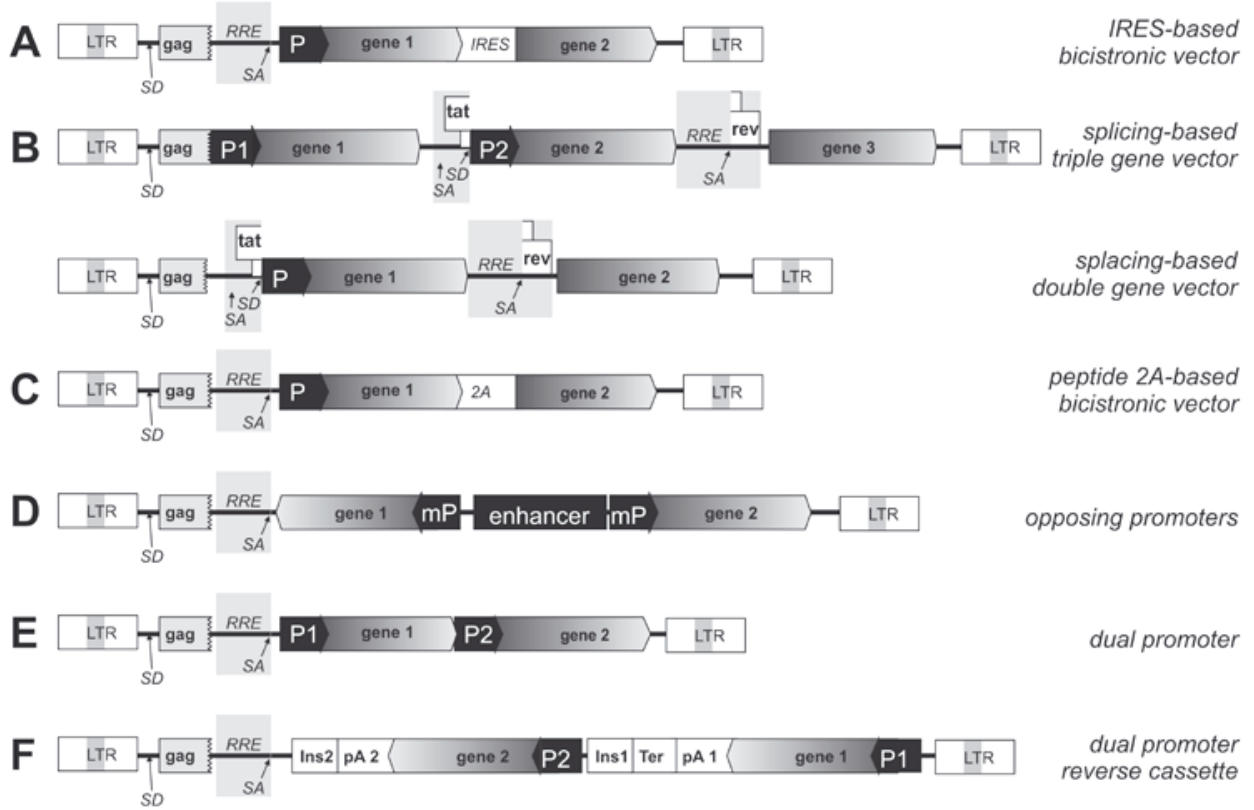

Figure 9. Transfer vectors for multigene expression.

A. Bicistronic vector based on IRES. Incorporation of IRES sequences from various viral genomes enables expression of more than one gene from single transcription unit. B. Multigene-expressing vectors based on splicing of mRNA. Multigene vector design includes utilization of one or two internal promoters (double- and triple gene cassette, respectively) and LTR-driven transcription of completely spliced mRNA (two or three independent transcriptional units). C. Bicistronic vector with peptide 2A sequence. Peptide 2A sequence enables fragmentation of nascent polyprotein through ribosomal skip mechanism, giving rise to two proteins encoded by single transcription unit. D. Expression of two genes from opposing promoters. Synthetic bidirectional promoter that consists of sequences of efficient promoter flanked by minimal promoter elements drives expression of divergent transgenes. E. Dual promoter-driven expression. Two transcriptional units are positioned in tandem forward orientation. F. Dual promoter-driven expression from reversed transcription cassettes. Two expression cassettes, inserted in reverse orientation with respect to LTRs, are separated by polyadenylation, terminator and insulator sequences.

Abbreviations: gag, 5' portion of gag gene containing dimerization/packaging signals; SD, splice donor site; SA, splice acceptor site; RRE, Rev response element; IRES, internal ribosome entry sequence; $2 \mathrm{~A}$, peptide $2 \mathrm{~A}$ sequence; $\mathrm{pA}$, polyadenylation signal; Ins, chromatin insulator; Ter, transcription terminator; P1, P2, internal promoters; $\mathrm{mP}$, minimal promoter. 
(2007) used integrase-defective lentiviral vectors to express ZFNs and template DNA in order to obtain gene correction in different cell types. Successful target gene editing was achieved in up to $39 \%$ of the cells. The authors also demonstrated the site-specific gene addition in up to $50 \%$ of the cells in the cell lines, albeit human embryonic stem (ES) cells were much less susceptible for the IDLV/ZNF-mediated DNA integration (less than $5 \%$ of the cells).

\section{METHODS FOR TRANSGENE EXPRESSION}

Lentivirus-based vectors have emerged as convenient and versatile tools for gene delivery and long-term controllable expression of genetic information in target cells and tissues in vitro and in vivo. Many studies, including in vivo experiments and gene therapy trials, may benefit from simultaneous expression of two or more genes from one vector. For example, delivery of the therapeutic gene(s) can be monitored by the presence of an accompanying product of marker gene(s), easily detected using enzymatic reactions or by its fluorescence. In ex vivo experiments, the population of transduced cells can be enriched based on marker gene expression (e.g., by drug resistance or fluorescence activated cell sorting, FACS). Last but not least, expression of a marker gene from an additional ORF helps in evaluation of the vector titer.

Some experimental and clinical settings require conditional expression of the gene of interest, which is possible using regulatable promoters. The most popular methods for controlled transgene expression are based on the presence of a specific activator or repressor in the same cell. Hence, for efficient and reliable control, co-expression of these two elements from one vector would be highly advantageous.

For genetic correction of metabolic disorders, both in animal models and clinical applications, tissue-specific expression from a lentiviral vector is one of the most attractive possibilities. As was mentioned in the previous sections, pseudotyping of the vector with a tissue-specific envelope glycoprotein was shown, in a number of experiments, to be effective in delivering the transgene to the desired cells. Another valuable possibility of target-restricted expression is offered by the use of tissue-specific promoters that are exclusively or primarily active in an environment of the specific cell.

\section{MULTIGENE VECTORS}

\section{IRES and splicing-based transgene expression}

The idea of co-expression of more than one gene from a single lentiviral vector has been real- ized by different approaches. Reiser et al. (2000) described several sets of constructs, including bicistronic vectors and multigene vectors, expressing up to three exogenous genes from two or three different transcriptional units. Bicistronic expression cassettes (Fig. 9A) utilize a single heterologous promoter driving two separate ORFs linked by an IRES sequence. Expression of the second gene depends on the strength of the promoter (the ability to produce long transcripts efficiently, often in a cell-dependent manner) and on the performance of the IRES (in this experimental setup, Gtx IRES, derived from the 5' UTR of the mRNA encoding the Gtx homeodomain protein, seems to be superior to the IRES derived from encephalomyocarditis virus, ECMV IRES; see also: Wang et al., 2005). A multigene vector design (Fig. 9B) involves utilization of one or two internal promoters (double- and triple gene cassette, respectively) and LTR-driven transcription of completely spliced mRNA. Thus, in contrast to a bicistronic vector, two or three independent transcriptional units are formed. Due to the fact that the last gene in the construct is driven by the LTR (fully spliced mRNA), its expression is Tat (but not Rev)-dependent. However, the LTR-driven expression levels varied between transduced cell lines. It was possibly caused by differences in cell-type-specific splicing kinetics (Reiser et al., 2000).

A similar vector design, employing IRES and natural HIV-1 splicing signals, was presented by Zhu et al. (2001b). Again, expression of the downstream gene was lower (by about $50 \%$ ), both in ECMV IRES-containing and splicing-based vectors. Interestingly, in the absence of Rev, expression of the second gene from spliced mRNA was relatively higher, probably due to increased amounts of doubly spliced RNA in the cytoplasm.

$\mathrm{Bi}-$ and multicistronic vectors effective both in vivo and in vitro were developed also by other groups (Stripecke et al., 2000; Mitta et al., 2002; Richard et al., 2004; Ben-Dor et al., 2006; Sangiolo et al., 2007; Lourenço et al., 2009) using different IRES sequences, including those derived from EMCV, hepatitis $\mathrm{C}$ virus (HCV) or poliovirus. An IRES was also included in the helper packaging construct used for the first clinical study in anti-HIV therapy (Lu et al., 2004; Levine et al., 2006). However, published data evidence that initiation of translation from an IRES is less efficient than from the $5^{\prime}$-end of the cap-containing transcript (Mizuguchi et al., 2000; Zhu et al., 2001b; Yu et al., 2003b; Osti et al., 2006; Chinnasamy et al., 2006; Khare et al., 2008; Ibrahimi et al., 2009).

Peptide 2A

To bypass the need for an IRES sequence, several groups used 2A peptide sequences (reviewed by Szymczak \& Vignali, 2005) (Fig. 9C) to 
construct bicistronic and polycistronic vectors effectively expressing multiple genes both in vitro and in vivo (Chinnasamy et al., 2006; 2009; Yang et al., 2008c; Hu et al., 2009a; Ibrahimi et al., 2009; Froelich et al., 2009). The advantage of using 2A peptide in the construction of bicistronic vectors is its ability to co-express both genes at nearly equal levels. The 2A, or similar peptides, are used by many positive strand RNA viruses to "cleave" their polyproteins in order to produce mature proteins. The 2A peptide, encoded by a sequence separating the $2 A$ and $2 B$ genes, exhibits a cleavage-like activity possibly through a ribosomal skip during translation. When this sequence is incorporated between heterologous ORFs, this apparent cleavage leaves about 18 amino acids of the 2A peptide at the C-terminus of the first protein and a single proline at the $\mathrm{N}$-terminus of the second one. Thus, a potential drawback of the use of 2A peptide is the possibility of inappropriate subcellular targeting of proteins (de Felipe \& Ryan, 2004), therefore further manipulations, e.g., addition of a furin cleavage site, are sometimes recommended (Yang et al., 2008c).

\section{Bidirectional synthetic promoters}

In search for efficient methods of multiple gene expression, Amendola et al. (2005) introduced synthetic bidirectional promoters to obtain divergent transcription of two mRNAs in the lentiviral vector. In the design of such synthetic bidirectional promoters a minimal core promoter is joined upstream to a strong promoter cloned in the opposite orientation (Fig. 9D). The rationale of this approach is that the upstream enhancer sequences in the efficient promoter, when flanked by minimal promoter elements, drive transcription in both directions. Lentiviral vectors containing such promoters, either constitutive or tissue-specific, enabled dual gene transfer into several tissues in vivo. Bidirectional vectors may be most useful for applications in which moderate transgene expression levels are desirable (Amendola et al., 2005; Ibrahimi et al., 2009).

The bidirectional concept system was later adapted for transcriptional amplification strategies (TASs) (Liu et al., 2008). In this strategy, the 5' cassette encoded an artificial transcriptional activator expressed from the minimal CMV promoter in reverse orientation. The downstream cassette allowed expression of the gene of interest from a tissue-specific promoter containing an upstream binding site for the transcriptional activator. In the in vitro and in vivo, results indicate that the TAS-amplified bidirectional promoters greatly increased the expression level of the reporter gene. Therefore, this system might be useful when weak tissue-specific promoters are considered. An attractive feature of this sys- tem is that the compact size of such promoters enables accommodation of larger expression cassettes.

\section{Dual promoter systems}

Problems with IRES-driven gene expression have led to the development of vector constructs containing two promoters (Fig. 9E). Two independent, constitutive promoters were employed to enable co-expression of two genes in transduced engrafting HSPCs and their progeny, as well as in other human cell types (Yu et al., 2003b). A similar system for reliable dual gene expression was described by Semple-Rowland et al. (2007). In their design two transgenes, each with its own tissue-specific promoter and arranged head-to-tail, were inserted into cHS4insulated lentiviral backbone and shared the same bGHpA sequence that replaced the 3' R/U5. Thus, the transcript encoded by the first gene was extended to include in its 3' UTR the promoter and coding regions of the second gene followed by the insulator sequence that was placed in the U3 region to enable duplication of the cHS4 at the $5^{\prime}$ end of the provirus. The dual-promoter vectors used in this study were able to target expression of two genes to a single cell type as well as to two different cell types within the same retinal tissue. Depending on the combination of the promoters used, one gene can be expressed in one cell type and the other in another type of cells, proving flexibility of the system.

However, the two transcriptional units positioned in this tandem forward orientation suffered from low level and/or inconsistent expression, possibly due to promoter interference or suppression, often expressed in a cell type-dependent manner. In an elegant study on dual promoter-driven gene expression, Tian and Andreadis (2009) reported on the construction of lentiviral vectors in which two expression cassettes, inserted in a reverse orientation with respect to LTRs, were separated by polyadenylation, terminator and insulator sequences (Fig. 9F). The combination of those elements eliminated promoter interference yielding high-level gene expression similar to that obtained by single-gene-encoding vectors. Analysis of co-expression of two fluorescent markers (EGFP and DsRed2) led to the construction of optimized vectors that performed equally well with several different promoters and cell types tested. The best results were obtained when the first gene was terminated with an SPA synthetic polyA signal, a pause G-rich sequence from the extension of $\beta$-actin gene $-\mathrm{T}_{\mathrm{actb}}$ terminator, and the cHS4 insulator. For optimal expression, the second ORF was followed by the thymidine kinase polyA (TKpA) signal and a synthetic sMAR8 insulator. Interestingly, some polyA sequences, e.g., SV40-derived one, reduced the viral titers significantly even when inserted in 

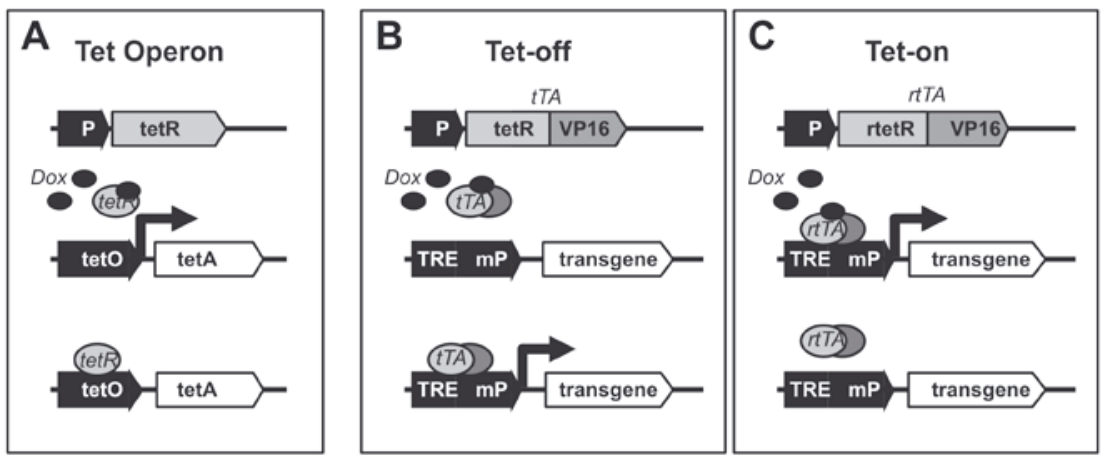

Figure 10. Tet operon-based system for regulatable gene expression from lentiviral vectors.

A. Bacterial Tet-resistance operon. In the presence of antibiotic, Tet-controlled repressor (tetR) dissociates from operator (tetO) and tetracycline resistance gene (tetA) is transcribed. B. Tet-off system. In the absence of doxycycline (Dox) Tetcontrolled transactivator (tTA, with HSV VP16 transactivation domain fused to tetR) binds TRE promoter (several tetO elements upstream of minimal promoter) and activates transcription. C. Tet-on system. In the presence of Dox reverse Tet-controlled transactivator (rtTA, mutant version of tTA) binds TRE promoter and activates transcription.

Abbreviations: $\mathrm{P}$, promoter; TRE, Tet-responsive element; $\mathrm{mP}$, minimal promoter.

reverse orientation, possibly due to their ability to terminate transcription in both directions.

In summary, the expression of two or more genes from one vector was demonstrated using various methods. The multiple gene expression was achieved either from single, polycistronic transcripts (with the IRES and 2A peptide sequences) or by generation of the independent transcription units (using splicing mechanism or multiple promoters). Additionally, the combination of different methods can be successfully applied. Nonetheless, each approach has its specific strengths and limitations, and these advantages and weaknesses must be considered when designing the vector for the particular needs.

\section{REGULATABLE EXPRESSION OF GENETIC IN- FORMATION}

For effective gene therapy both efficient delivery of therapeutic genes to the target cells and their regulatable expression are desired. The earliest regulatable systems, based on natural inducible promoters, were disappointing because of a high basal expression, weak induction levels, and pleiotropic effects often exerted by their control elements on mammalian cells. To overcome those limitations chimeric regulatable systems were introduced. This novel approach utilized various prokaryotic and eukaryotic elements incorporated into promoters and offered better control of the transgene than could be achieved with natural inducible promoters. To date, several systems for gene expression in mammalian cells have been described that use exogenous drugs, including antibiotics, steroid hormones, and dimerizers, to control transgene expression (reviewed in: Agha-Mohammadi \& Lotze, 2000; Clackson, 2000; Gossen \& Bujard, 2002; Toniatti et al., 2004; Goverdhana et al., 2005). However, many of those systems still show either leakiness (i.e., undesired transgene expression when the inducer is absent) or poor activation (i.e., insufficient transgene expression in the presence of the inducer) and, in order to alleviate these problems, further improvement of the regulatable systems is a must.

Optimized tetracycline-responsive promoters

Among other systems, tetracycline (Tet)-responsive promoters have the most promising characteristics and the Tet-based systems are most widely used in lentiviral vectors. The system is entirely prokaryotic and employs components of E. coli Tetresistance operon (derived from transposon Tn10) (Fig. 10A) (for review see Corbel \& Rossi, 2002). Due to this, elements of the Tet-based system should not exert pleiotropic effects in eukaryotic cells. Another advantage of using Tet-responsive promoters is that their effector molecule, Tet, or its synthetic derivative, doxycycline (Dox), can be administered orally and are able to penetrate all tissues (also pass the blood/brain barrier). Importantly, Dox functions in the Tet-based system at levels below those required for bacteriostatic activity and has a relatively short half-life, which allows rapid response following withdrawal of the antibiotic (Baron \& Bujard, 2000). This response does not exhibit a threshold characteristic and is dose-dependent (Gossen \& Bujard, 1992; Gossen et al., 1995; Kringstein et al., 1998).

In the original Tet-off system developed by Bujard and co-workers (Gossen \& Bujard, 1992), a Tet-controlled transactivator (tTA) binds the Tet-responsive element (TRE) and induces gene expression in the absence of Tet or Dox (Fig. 10B). The TRE consists of seven tandem copies of Tet operator (tetO) sequences (each $18 \mathrm{bp}$ long) and is placed upstream of the CMV minimal promoter (TRE/CMV). tTA is a chimeric protein in which bacterial Tet re- 
pressor (tetR) is fused to the HSV VP16 transactivation domain. In contrast, in the Tet-on system, the binding of the reverse Tet-controlled transactivator (rtTA), a mutated version of tTA, and the resultant gene expression is induced by Dox (Fig. 10C) (Gossen et al., 1995). Hence, both Tet-based systems require that two components, an inducible transgene and the transactivator, be present in the same cell.

Several groups described inducible Lentivirus vectors based on the Tet-off system (Kafri et al., 2000; Vigna et al., 2002; Régulier et al., 2002; 2003). Their results show very high induction levels - more than 500-fold above background. Unfortunately, these background levels in the repressed state were also unacceptably high. Nonetheless, the main limitation of this system, especially in clinical settings, is that constant presence of Dox is required to suppress transgene expression. In contrast, the Tet-on system requires the presence of the drug only to activate gene expression. Reiser et al. (2000) and Johansen et al. (2002) designed HIV-1-based Teton vectors involving the original rtTA. However, background expression levels were high with this system, which could be, at least partially, attributed to transcription from the $5^{\prime}$ LTR or cryptic promoters present in the neighboring chromatin. Moreover, transcription activation after addition of the inducer was poor.

Further attempts to improve Tet-inducible vectors have been made over the years. Koponen et al. (2003) used a SIN Lentivirus (133 bp deletion in U3 region) bearing a transgene controlled by a minimal CMV promoter coupled with TRE in combination with a second vector encoding enhanced rtTA2 ${ }^{\mathrm{S}}$-M2 transactivator (Urlinger et al., 2000). As one could expect, this transactivator performed much better than the original rtTA (130-fold induction), possibly due to its improved stability and enhanced sensitivity to Dox. However, the high background expression persisted (Koponen et al., 2003).

Pluta et al. (2005) tested several different approaches toward reduction of the system leakiness and improvement of activation. First, direct comparison of the performance of various transactivators confirmed the superiority of $\mathrm{rtTA}^{\mathrm{S}} \mathrm{S} \mathrm{M} 2$ over rtTA, rTE4d38, and $\mathrm{rtTA}_{2}{ }^{\mathrm{S}}$-S2. Second, to minimize background expression, as much as $400 \mathrm{bp}$ was deleted from the U3 sequence $(\Delta \mathrm{U} 3)$ present in the proviral LTRs. Third, a $1.2 \mathrm{~kb}$-long cHS4 was incorporated in place of the U3 deletion in the $3^{\prime}$ LTR in order to shield the expression cassette from the influence of surrounding host chromatin enhancers (after duplication of the cHS4-containing $\triangle \mathrm{U} 3$ LTR). Additionally, to increase the level of activated transgene expression and to reduce background transcription second-generation TREs, in which the distances and positions of consecutive tetO sequence elements had been optimized, were used. One of those improved TREs, TRE/Tight, was commercially available (Clontech), the other one, TRE/Pitt had been described by Agha-Mohammadi et al. (2004). In TRE/Pitt, the central bases of eight tetO sequences are separated by $36 \mathrm{bp}$ ( 3.5 helical turns) and the TATA box of the minimal CMV promoter is placed $10 \mathrm{bp}$ (1 helical turn) downstream of the first tetO element. Deletion in the U3 region alone did not significantly reduce the background transgene expression and it was concluded that the regular TRE/CMV promoter in the context of lentiviral vectors is leaky in the absence of both Dox and transactivator. As expected, the insulator sequences flanking the regulatable expression cassette reduced expression in the absence of Dox, but the induction levels were not as high as without cHS4. In turn, replacing TRE/CMV with the second-generation TREs decreased the leakiness and improved activation. The lowered basal expression might be explained by the absence of functional sequences that are present between the heptameric tetO sequences in the original TRE (Rang \& Will, 2000). Background expression levels were lower with TRE/Tight than with TRE/Pitt, but induced expression levels in the presence of Dox were much

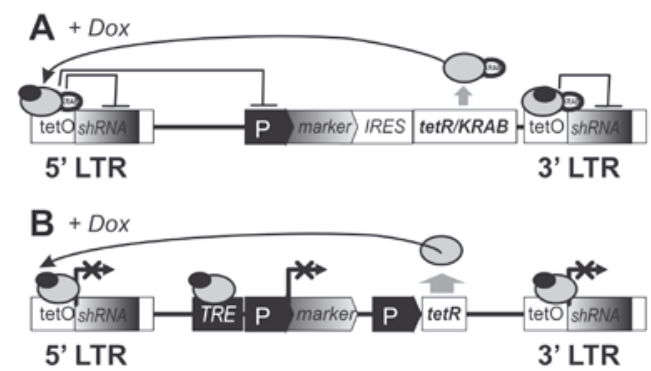

Figure 11. Single-vector system for controllable expression of shRNA and transgene.

A. System equipped with negative autoregulatory feedback loop. In repressed state (Dox present), Tet-controlled repressor, fused to KRAB protein silencer domain, binds to tetO sequences and blocks transcription of shRNA from RNAP III promoter. Simultaneously, due to KRAB chromatin silencing effect, transcription from internal RNAP II promoter, driving expression of marker gene and repressor (from IRES sequence), is also stopped. Thus, concentration of effector protein will decrease over time to a point where repression is partially relieved. Resulting re-synthesis of tetR-KRAB will repress system again. B. Simplified system based on bare tetR repressor. This system utilizes regular Tet-controlled repressor synthesized from independent constitutive promoter. Both regulatable promoters, RNAP III - driving synthesis of shRNA, and RNAP II for transgene expression, contain repressor-binding sequences. Continuous production of tetR ensures efficient repression of transcription in the presence of Dox.

Abbreviations: Dox, doxycycline; tetO, tetracycline operator sequence; shRNA, short hairpin RNA; P, internal promoter; IRES, internal ribosome entry sequence; TRE, Tetresponsive element; tetR, Tet-controlled repressor; tetR$\mathrm{KRAB}$, Tet-controlled repressor fused to KRAB protein silencer domain. 
higher with TRE/Pitt than with TRE/Tight (522-fold and 90 -fold induction, respectively). For the original TRE/CMV, induction was only 31-fold above background, whereas leakiness was 2.4 and 5.4 times higher than for TRE/Pitt and TRE/Tight, respectively. This regulatable system was then successfully tested in vivo (Pluta et al., 2005).

Since binary vector systems were used for Tet-controlled gene expression, the undesired basal expression might be also attributed to the fact that the regulatory protein was not present in all cells. This problem can be bypassed by selection (sorting) of doubly transduced cells, but for in vivo studies this option is not available. The TRE/Tight promoter has been recently successfully used for construction of a regulatable single-vector system and this Tet-off platform (based on a modified tTA) was applied for an in vivo study in rat neurons (Hioki et al., 2009). The principle of the design was that the marker gene (with its polyA signal) was placed under the control of TRE in reverse orientation and the transactivator was expressed from a tissue-specific promoter in forward orientation. This system supported a 40-times higher expression of the marker gene in neurons in vivo compared to a regular lentiviral vector (without the TRE promoter and in the absence of tTA). Moreover, the delivery of the transgene to target cells was about 7-8 times more efficient when the combined vector was used instead of its twovector counterpart.

\section{Regulatable expression of siRNAs}

Another approach to controlling transgene expression using a Tet-based system employs Tet-controlled transcriptional repressors (tTS). In tTS tetR sequences were fused to the Krüppel-associated box (KRAB) protein domain of human KOX1, a Krüppeltype zinc finger factor (Deuschle et al., 1995; Moosmann et al., 1997). KRAB is an approximately 75amino acid transcriptional repression module which can suppress both polymerase II- and polymerase III (RNAP II and RNAP III)-mediated transcription within a distance of $2-3 \mathrm{~kb}$ from its binding site, presumably by triggering the formation of heterochromatin (Margolin et al., 1994; Senatore et al., 1999; Ryan et al., 1999).

Wiznerowicz and Trono (2003) used this system for conditional co-expression of a marker gene and small interfering RNA (siRNA) from a lentiviral vector (for recent reviews on RNA interference pathway, RNAi, and lentiviral vectors as a delivery tool, see: Morris \& Rossi, 2006; Scherr \& Eder, 2007; Llano et al., 2009; Miest et al., 2009; Manjunath et al., 2009). In their design, both the tetO site and the small hairpin RNA (shRNA) sequence driven by an RNAP III promoter, are located in the U3 region and are duplicated in the integrated provirus. This

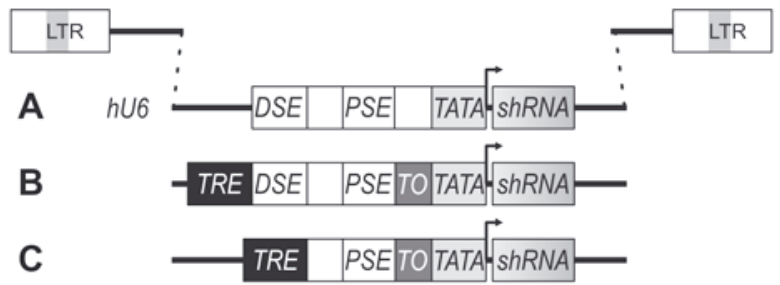

Figure 12. Adaptation of RNAP III promoter for regulatable shRNA expression from lentiviral vector.

A. Structure of human U6 promoter (hU6). B. hU6 promoter modified by insertion of single tetO operator sequence between PSE and TATA box and second-generation TRE sequence upstream of DSE. This construct facilitates tight expression control using doxycycline and tetR-KRAB repressor. C. DSE sequence of hU6 promoter, containing single tetO operator between PSE and TATA box, is replaced with second-generation TRE. This enables Tet-controlled activation of transcription with reverse transactivator bearing Sp1 transactivation domain.

Abbreviations: LTR, long terminal repeat; DSE, distal sequence element; PSE, proximal sequence element; TATA, TATA box; shRNA, short hairpin RNA; TO, tetO operator; TRE, Tet-responsive element.

allowed production of doubled amounts of siRNA in activated state (Dox present). In the absence of the effector tTS tightly suppressed expression of the shRNA from the promoter adjacent to the tetO site. However, with such a vector, where internal RNAP II promoter was placed further away from the tTS binding sites, leaky expression of the marker gene was observed (Zhou et al., 2007). This could be avoided by placing TRE closer to the promoter. Optimized vectors were less leaky and activation of the system required only low concentrations of Dox (5 $\mathrm{ng} / \mathrm{ml})$. However, addition of Dox did not uniformly induce the transgene expression and "locked" suppression was observed in about $50 \%$ of transduced cells (Zhou et al., 2007). This may suggest a serious limitation for the use of such chromatin-modulating suppressors.

Based on their original design (Wiznerowicz \& Trono, 2003), Trono and co-workers reported an elegant single-vector system for controllable expression of shRNA and a transgene (Szulc et al., 2006). The major modification was the insertion of an IRES/tTS cassette downstream of the marker gene (Fig. 11A). Thus, in the activated state, expression from the internal promoter leads to production of transgene, tTS and siRNA. In the repressed state, tTS binds to the tetO site near the RNAP III promoter/shRNA cassette in the vector LTRs and blocks transcription from both the RNAP III and the internal RNAP II promoter. The system comes in two flavors: Tet-on and Tet-off, depending on which tetR is used for creation of the fusion repressor (tTS or rtTS, respectively), so Dox can be used in both ways. Once the internal promoter is shut down, the concentration of 
the effector decreases to a point when the repression is partially relieved. The resulting re-synthesis of tTS represses system again. This negative autoregulatory feedback loop (Fig. 11A) reduces the amount of tTS required for suppression. The system exhibited low leakiness in the repressed state and robust inducibility upon activation in both cell lines and primary cells as well as in mouse in vivo models.

Later, a similar system was described, albeit a much simpler one - without the autoregulatory features (Fig. 11B) (Zhang et al., 2007a). The design was based on an unmodified tetR repressor expressed from a constitutive RNAP II promoter. Due to the lack of the KRAB silencer, tetR repressed only the tetO-equipped RNAP III promoter driving shRNA. Thus, the constantly high level of tetR ensured that the inducible promoter was effectively turned off. Moreover, this repression depended on simple TetR-RNAP III interactions, not on a chromatin remodeling mechanism. However, one possible limitation for such single-vector designs is the restricted payload of Lentivirus-based vectors. Also the proximity of the promoters, one driving the effector protein and the other - the gene of interest or siRNA, might result in transcriptional interference, therefore binary vector systems that separate these two expression cassettes are still popular.

Usually the RNAP III promoters, H1 or U6 (Fig. 12A), adopted to express siRNA in a Tet-regulatable manner, harbor a single tetO element inserted between the proximal sequence element (PSE) and TATA box, so it is tempting to introduce multiple tetO sequences in order to enhance the effect of regulatory protein binding. However, due to the very compact structure of the promoter such manipulations often lead to partial or complete abrogation of its function (Chen et al., 2003; Lin et al., 2004; Zhang et al., 2007a). Other attempts to improve the performance of a regulatable RNAP III promoters were made by Pluta et al. (2007). In this case the human U6 promoter was engineered by insertion of a TRE sequence derived from the aforementioned TRE/Pitt promoter upstream of the distal sequence element (DSE). Therefore, the regulatable promoter contains a single tetO sequence between PSE and TATA plus additional eight tetO elements at the $5^{\prime}$ end (Fig. 12B). Using a SIN lentiviral system and the new Tet-responsive promoter the authors demonstrated a fully reversible decrease of expression of a biologically relevant target (CXCR4) to about $10 \%$ of a control experiment (absence of the siRNA) without any noticeable leakiness (no target gene silencing) in the absence of inducer (Dox). Notably, this robust silencing was achieved with relatively low multiplicities of infection (MOIs) (MOI of 25 for both tTS- and CXCR4 shRNA-expressing viruses) in a mixed cell population (unsorted cells).
In the same study the possibility of transactivation using modified RNAP III promoters was investigated. To make this "reverse" system, the DSE in the human U6 promoter, which is responsible for RNAP III transcription regulation (Murphy, 1997) was replaced with a second-generation TRE (Fig. 12C) in order to make it responsive to an Sp1-containing transactivator that preferentially transactivates RNAP III-type promoters (Das et al., 1995; Strom et al., 1996). In the presence of Dox, the modified rtTA with the VP16 domain replaced by an Sp1 domain was found to bind to the TRE and enhance transcription from the modified U6 promoter (Pluta et al., 2007). Similar approaches have also been reported by others: an ecdysone-inducible hybrid RNAP III promoter combined with a GAL4/ Oct $2{ }^{\mathrm{Q}}(\mathrm{Q} \rightarrow \mathrm{A})$ transactivator (comprising the DNA binding domain of the yeast positive regulatory protein GAL4 and an artificial transactivation domain derived from the human transcription factor Oct-2) (Gupta et al., 2004) or the TRE/U6 promoter responsive to $\operatorname{rtTA} / \operatorname{Oct}_{2} \mathrm{Q}(\mathrm{Q} \rightarrow \mathrm{A}$ ) (Amar et al., 2006). However, all these interesting systems suffer from noticeable leakiness (unless transduced cells are sorted) and their fine-tuning requires more effort.

Tet-responsive RNAP II promoters have also been used for regulated siRNA expression. Stegmeier et al. (2005) described a system with Tet-regulated expression of a microRNA-like short hairpin RNA (miR-shRNA) using improved TRE promoters. Since microRNA-derived siRNA can be processed from longer precursors than regular shRNA sequences, it allows the use of RNAP II promoters for its transcription. In the presented design, miR-shRNA was expressed in a fusion with a transgene sequence or the marker gene was translated from an IRES following the miR-shRNA sequence. Hence, expression of the marker gene was only visible in cells in which siRNA-directed target gene silencing was induced. Although this system performed well at low MOIs (sorted cells), it relays on cells stably producing the transactivator.

More recently, a single-vector design that uses TRE to drive miR-shRNA has been reported (Shin et al., 2006). In this construct the TRE/microRNA cassette is followed by a constitutive promoter driving expression of a bicistronic rtTA/IRES/marker gene cassette. Due to the constitutive expression of the transactivator and the reporter protein, all cells transduced with the all-in-one virus can be traced even when production of siRNA is not activated. Notably, this system is equipped with a positive feedback loop: in the activated state, translation of the longer transcript expressed from the TRE, containing also the rtTA and marker gene sequences, increases the amount of transactivator and amplifies the marker signal in cells with activated gene silencing. 


\section{Cre/loxP transcriptional switch}

Another method for inducible siRNA expression is based on Cre/loxP recombination. This technique takes advantage of the ability of bacteriophage P1 Cre recombinase to delete from target DNA sequences flanked with two loxP sites. Cre-mediated excision of entire provirus (loxP sites inserted into the U3 region) has been used previously to turn off transgene expression at a desired time (Salmon et al., 2000b; Mitta et al., 2002; Chang \& Zaiss, 2003). If such loxP-flanked sequences ("stuffer sequences") inserted between the promoter and shRNA or in the loop between the sense and antisense strands of shR$\mathrm{NA}$, and hence hindering transcription, are removed, expression of functional siRNA begins. The Cre recombinase can be delivered to target cells by means of Lentivirus (Tiscornia et al., 2004; Heldt et al., 2007) or loxP/shRNA transgenic mice can be crossed with tissue-specific Cre-expressing mice (Ventura et al., 2004; Yu \& McMahon, 2006; Shukla et al., 2007; Hitz et al., 2007; Steuber-Buchberger et al., 2008; Stern et al., 2008; Hitz et al., 2009).

Generally, because this mode of transcription activation is irreversible, such strategy is rather suited to study gene downregulation in transgenic animals, especially those genes whose permanent knockdown could result in embryonic lethality. Since many strains of cell- and tissue-specific Cre-expressing mice are currently available, this method is also useful for the analysis of temporal and tissue-specific effects of individual genes expression (Hitz et al., 2007; SteuberBuchberger et al., 2008; Hitz et al., 2009).

In summary, various systems for the regulatable gene expression have been used in a context of Lentivirus-based vectors. However, the Tet-responsive promoters are the most promising in terms of their high inducibility and low leakiness. Further improvements of the elements of this system should result in the creation of clinically relevant technology for the tight control of gene expression.

\section{TISSUE-SPECIFIC PROMOTERS}

Strong, ubiquitously active promoters, such as those of $\mathrm{CMV}$, mouse phosphoglycerate kinase-1 (PGK-1), human translation elongation factor 1- $\alpha$ (EF1- $\alpha)$, human ubiquitin $\mathrm{C}(\mathrm{hUbC})$ or the hybrid CMV enhancer/chicken $\beta$-actin promoter (CAG), are widely used to express transgenes from integrated lentiviral vectors. Yet limitations of these promoters in the in vivo gene transfer have prompted a search for tissue-specific promoters and enhancers. Certain ubiquitously active promoters have recently been found to be silenced following transplantation of ex vivo transduced cells (Chang et al., 2006; Zhang et al., 2007b), in cloned feline embryos (Gómez et al., 2009) and in human ES cells (Xia et al., 2007). Moreover, the trans- gene expression driven by those promoters may provoke transgene-specific cellular and humoral immune responses, leading to the clearance of transduced cells from organs (Follenzi et al., 2004; Limberis et al., 2009).

One possible reason for this situation is unwanted transgene expression in antigen presenting cells (APCs), found in all tissues, when a viral vector is administered in vivo. It has been demonstrated that expression of the transgene (tumor antigen) in DCs leads to expansion and activation of antigenspecific T cells (Cui et al., 2003). Moreover, Brown et al. (2007a) clearly showed that activation of IFN $\alpha \beta$ is responsible for the rapid clearance of transduced cells from the liver. After in vivo administration of lentiviral vectors, induction of the type I interferon signaling response in DCs occurred within few hours and was independent of the envelope pseudotype. Instead, the activation of the innate antiviral response was concomitant with the presence of the reverse-transcribed vector DNA. Thus, before the development of an adaptive immune response against transgene transduction of hepatocytes was blocked at early stages of infection by induction of the IFNs.

Transcriptional targeting of the vector by means of tissue-specific promoters in combination with pseudotyping (see section on Targeting Lentiviral Vectors by Pseudotyping) in order to restrict transgene expression to desired cells and tissues provides a solution for some of the aforementioned problems. Examples of tissue-specific promoters and enhancers used to express transgenes from Lentivirus-based vectors are presented in Table 2.

While tissue-specific promoters used in a viral vector confer selectivity, their activity is usually weak and the level of transgene expression may be too low for detection or to exert the expected effect on the target cell. To address this issue, a CMV-derived enhancer was used in combination with several tissue-restricted promoters (Gruh et al., 2008). This resulted in a multiple-log increase in marker gene expression compared to vectors lacking the enhancer, without, however, substantially compromising its tissue-specificity.

Another way to increase the level of tissuespecific expression is the transcriptional amplification strategy mentioned in the section dealing with Bidirectional Synthetic Promoters. While Liu et al. (2008) used the CMV promoter to drive the synthesis of a transactivator (GAL4BDp65; with the transcriptional activation domain of the NF- $\kappa B$ p 65 protein) and a GAL4-responsive tissue-specific promoter to drive the reporter gene, Shaw et al. (2009) employed a reverse strategy for their TAS. In contrast to the design of Liu et al. (2008), the GAL4VP16 transactivator was transcribed from a tissue-specific promoter, whereas the gene of interest was placed under the control of a GAL4-responsive element. No 
off-target expression was observed and the inducible promoter was able to increase the level of gene expression five-eight-fold over that obtainable with a standard promoter vector.

\section{PRODUCTION OF LENTIVIRAL VECTORS - PRACTICAL GUIDE}

The production and handling of Lentivirusbased vectors should be carried out with special at- tention paid to personal and environmental safety, using proper procedures and biosafety containment (i.e., Biosafety Level 2, BL2, containment; for more information see List of websites [10 and Appendix G-III-L,N therein]).

Protocols for production and concentration of lentiviral vectors are widely available in the literature. Successively modified procedures are regularly published by Reiser and co-workers (Reiser, 2000; Marino et al., 2003; Kuroda et al., 2009; Kutner et al., 2009a; 2009b) and by others (Karolewski et al.,

Table 2. Selected tissue-specific promoters and enhancers used in Lentivirus-based vector context

\begin{tabular}{|c|c|c|c|c|}
\hline $\begin{array}{l}\text { Targeted } \\
\text { tissue }\end{array}$ & Promoter & Source & Comments & References \\
\hline \multirow{6}{*}{ 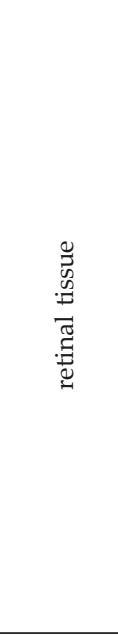 } & mouse CD44 & $\begin{array}{l}\text { gene encoding transmembrane glycopro- } \\
\text { tein and cell surface } \\
\text { receptor for hyaluronic acid }\end{array}$ & active in glial Müller cells & $\begin{array}{l}\text { Greenberg et al., } \\
2007\end{array}$ \\
\hline & $\begin{array}{l}\text { human and mouse } \\
\text { GFAP }\end{array}$ & $\begin{array}{l}\text { gene encoding human and mouse glial } \\
\text { fibrillary acidic protein }\end{array}$ & active in glial Müller cells & $\begin{array}{l}\text { Greenberg et al., } \\
2007\end{array}$ \\
\hline & mouse VIM & $\begin{array}{l}\text { gene encoding vimentin - major subunit } \\
\text { protein of intermediate filaments }\end{array}$ & active in glial Müller cells & $\begin{array}{l}\text { Greenberg et al., } \\
2007\end{array}$ \\
\hline & IRPB1783 & $\begin{array}{l}\text { gene encoding interphotoreceptor retino- } \\
\text { id binding protein }\end{array}$ & active in cone cells & $\begin{array}{l}\text { Semple-Rowland et } \\
\text { al., } 2007\end{array}$ \\
\hline & GCAP292 & $\begin{array}{l}\text { gene encoding guanylate cyclase activa- } \\
\text { ting protein } 1\end{array}$ & active in cone cells & $\begin{array}{l}\text { Semple-Rowland et } \\
\text { al., } 2007\end{array}$ \\
\hline & mOP500 & rhodopsin gene & active in rod cells & $\begin{array}{l}\text { Semple-Rowland et } \\
\text { al., } 2007\end{array}$ \\
\hline \multirow{4}{*}{ 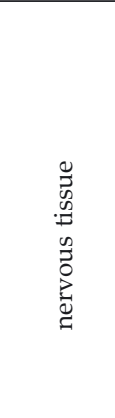 } & CamKII & $\begin{array}{l}\text { gene encoding calcium/calmodulin-de- } \\
\text { pendent protein kinase II }\end{array}$ & $\begin{array}{l}\text { active in neurons of adult } \\
\text { forebrain }\end{array}$ & $\begin{array}{l}\text { van Hooijdonk et } \\
\text { al., } 2009\end{array}$ \\
\hline & SYN & synapsin 1 phosphoprotein gene & $\begin{array}{l}\text { active in some regions of } \\
\text { hippocampus }\end{array}$ & $\begin{array}{l}\text { Kuroda et al., 2008; } \\
\text { van Hooijdonk et } \\
\text { al., } 2009\end{array}$ \\
\hline & NSEp & gene encoding neuron-specific enolase & $\begin{array}{l}\text { active in brain striatum } \\
\text { and hippocampus }\end{array}$ & Lai \& Brady, 2002 \\
\hline & $\mathrm{GfaABC}_{1} \mathrm{D}$ & glial fibrillary acidic protein (GFAP) gene & active in glia in CNS & Liu et al., 2008 \\
\hline$\beta$-cells & insulin & human insulin gene & $\begin{array}{l}\text { active in } \beta \text {-cells and mu- } \\
\text { rine insulinoma cell lines }\end{array}$ & Shaw et al., 2009 \\
\hline \multirow{4}{*}{ 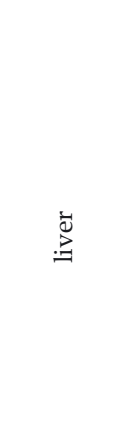 } & ALB & albumin gene & active in hepatocytes & Follenzi et al., 2004 \\
\hline & ET & synthetic promoter & $\begin{array}{l}\text { more active than ALB; } \\
\text { minor activity in spleen }\end{array}$ & Brown et al., 2007 \\
\hline & ApoA-II & human apolipoprotein A-II gene & $\begin{array}{l}\text { inducible by different } \\
\text { factors, including fibrates, } \\
\text { statins, etc. }\end{array}$ & Dagher et al., 2009 \\
\hline & $\alpha 1-\mathrm{AT}$ & human $\alpha 1$-antitrypsin gene & $\begin{array}{l}\text { active in hepatocyte pro- } \\
\text { genitors and hepatocytes }\end{array}$ & Duan et al., 2007 \\
\hline
\end{tabular}




\begin{tabular}{|c|c|c|c|c|}
\hline \multirow{5}{*}{ 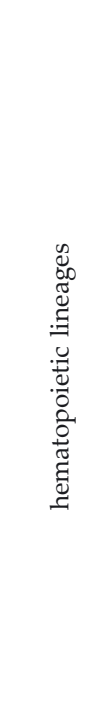 } & WASp & $\begin{array}{l}\text { gene encoding Wiskott-Aldrich syndrome } \\
\text { protein }\end{array}$ & $\begin{array}{l}\text { active in T cells, B cells, } \\
\text { dendritic cells (DCs) and } \\
\text { CD34+ progenitor cells }\end{array}$ & Charrier et al., 2007 \\
\hline & Tie2 & $\begin{array}{l}\text { gene encoding cell-surface angiopoietins } \\
\text { receptor }\end{array}$ & $\begin{array}{l}\text { active in endothelial cells } \\
\text { (ECs) and monocytes }\end{array}$ & $\begin{array}{l}\text { De Palma et al., } \\
\text { 2003; De Palma et } \\
\text { al., } 2008\end{array}$ \\
\hline & $\begin{array}{l}\text { promoters: } \\
\text { ankyrin- } 1, \alpha \text {-spectrin, } \\
\beta \text {-globin, } \zeta \text {-globin } \\
\text { enhancers: } \\
\text { GATA- } 1, \beta \text {-globin } \\
\text { LCR, intron I8, } \\
\alpha \text {-globin HS40, } \\
\gamma \text {-globin intron } \\
\end{array}$ & erythroid-specific genes & $\begin{array}{l}\text { active in erythroid line- } \\
\text { ages }\end{array}$ & $\begin{array}{l}\text { Moreau-Gaudry et } \\
\text { al., 2001; Hanawa } \\
\text { et al., } 2002\end{array}$ \\
\hline & HLA-DR $\alpha$ & $\begin{array}{l}\text { gene encoding } \alpha \text { subunit of human leu- } \\
\text { kocyte antigen DR }\end{array}$ & $\begin{array}{l}\text { active in antigen presen- } \\
\text { ting cells (APCs) and DCs }\end{array}$ & Cui et al., 2002 \\
\hline & proximal $l c k$ & $\begin{array}{l}\text { gene encoding T-cell-specific protoonco- } \\
\text { gene, lck }\end{array}$ & active in $T$ cells & Lois et al., 2002 \\
\hline \multirow{2}{*}{ 䒕 } & ANF & $\begin{array}{l}\text { gene encoding human atrial natriuretic } \\
\text { factor }\end{array}$ & active in cardiomyocytes & Gruh et al., 2008 \\
\hline & MLC2v & $\begin{array}{l}\text { gene encoding human ventricular myosin } \\
\text { light chain }\end{array}$ & active in cardiomyocytes & Gruh et al., 2008 \\
\hline lung & SP-C & $\begin{array}{l}\text { type II alveolar epithelial cell (AT-2)-spe- } \\
\text { cific human surfactant protein C gene }\end{array}$ & active in AT-2 cells & Gruh et al., 2008 \\
\hline prostate & PSAp & gene encoding prostate-specific antigen & - & $\begin{array}{l}\text { Yu et al., 2001; } \\
\text { Zheng et al., } 2003\end{array}$ \\
\hline muscle & myogenin & - & - & Lois et al., 2002 \\
\hline oviduct & OVA & chicken ovalbumin & $\begin{array}{l}\text { marker gene exclusively } \\
\text { expressed in transgenic } \\
\text { hen oviduct }\end{array}$ & Lillico et al., 2007 \\
\hline
\end{tabular}

2003; Sena-Esteves et al., 2004; Mitta et al., 2005; al Yacoub et al., 2007; Segura et al., 2007; Ansorge et al., 2009; Bagnis et al., 2009). Moreover, a video tutorial for manufacturing retrovirus-based vectors by transient transfection of $293 \mathrm{~T}$ cells can be found on-line (see List of websites [11]) (Gavrilescu \& Van Etten, 2007). A schematic protocol for production of Lentivirus-based vector by transient transfection using DNA/calcium phosphate coprecipitation is presented in Table 3.

In this review, we will point out critical steps in the protocol and briefly describe recent advances in this field.

\section{PRODUCTION OF LENTIVIRAL VECTORS BY TRANSIENT TRANSFECTION}

The traditional method for lentiviral vector production relies on efficient cotransfection of producer cells, namely human embryonic kidney 293T (HEK 293T) cells (ATCC No. CRL-11268), with three (second-generation packaging system) or four (third- generation packaging system) plasmids: helper(s), envelope, and vector (Fig. 7; see section on Evolution of vector design concept).

Alternatively, viral vector production can be accomplished by transfer vector plasmid transfection of a producer cell line stably expressing packaging proteins and an envelope glycoprotein (reviewed by Cockrell \& Kafri, 2007; see section on Envelope Expressing Cassette). This might reduce the risk of recombination between the transfected plasmids and minimize the problem of carrying over plasmid DNA in the vector preparation. However, this method is hampered by several limitations: the long time required to develop a stable cell line expressing all necessary vector components in a regulatable manner (due to possible cytotoxicity of some proteins, especially viral protease, Rev and VSV-G), the need for manufacturing designated cell lines for each desired vector pseudotype, low vector titers and, last but not least, genetic instability over long culture periods.

An interesting alternative for transient plasmid transfection is baculovirus-based Lentivirus 
Table 3. Schematic protocol for production of Lentivirus-based vector by transient transfection using DNA/calcium phosphate coprecipitation

\begin{tabular}{|c|c|c|}
\hline Day & Step & Operation \\
\hline 1st & plating cells & $\begin{array}{l}\text { plate desired number of thoroughly separated HEK } 293 \mathrm{~T} \text { cells (to get } 50-80 \% \text { confluence on } \\
\text { the next day) }\end{array}$ \\
\hline 2nd (evening) & transfection & $\begin{array}{l}\text { 1. add chloroquine to culture medium (to } 25 \mu \mathrm{M} \text { final concentration) } \\
\text { 2. prepare transfection mix: } \\
\text { a. mix water, plasmid DNAs and } \mathrm{CaCl}_{2} \text { (to } 250 \mathrm{mM} \text { final concentration) } \\
\text { b. add mixture drop-wise to equal volume of } 2 \times \mathrm{HBS} \mathrm{pH} 7.0-7.3 \text { (fresh batch must } \\
\text { always be tested for transfection efficiency) while vortexing (gently) } \\
\text { 3. pipette DNA/calcium/HBS mix drop-wise onto cultured cells, swing plate to evenly } \\
\text { distribute precipitate }\end{array}$ \\
\hline 3rd (morning) & $\begin{array}{l}\text { culture mainte- } \\
\text { nance }\end{array}$ & change medium \\
\hline 4 th & $\begin{array}{l}\text { transfection pro- } \\
\text { gress monitoring }\end{array}$ & $\begin{array}{l}\text { check cells with fluorescence microscope: if transfer vector contains fluorescent marker gene } \\
\text { - majority of cells should express it; if envelope vector codes for VSV-G - cell membranes } \\
\text { should start to fuse }\end{array}$ \\
\hline 5 th & collecting virus & $\begin{array}{l}\text { harvest virus-containing medium, filter cell debris through } 0.45 \mu \mathrm{m} \text { polysulfone filter, store at } \\
-80^{\circ} \mathrm{C} \text { or concentrate directly }\end{array}$ \\
\hline
\end{tabular}

production (Lesch et al., 2008). In this method, four recombinant baculoviruses deliver all necessary lentiviral vector components to infected 293T cells. This technique takes advantage of the fast and easy production of baculoviruses, efficient transduction of mammalian cells and the safety of baculoviruses that do not replicate in vertebrate cells.

\section{Cell lines and plasmid DNA}

The 293T cell line has some unique features that make it crucial for lentiviral vector production. The cells are highly transfectable $(70-90 \%$ of positive cells) and additionally express SV40 T-large antigen, which enables replication of plasmids containing SV40 ori of replication (Soneoka et al., 1995). The use of another HEK cell line derivative, 293E cells, expressing Epstein-Barr virus (EBV) nuclear antigen-1 (EBNA-1), has also been reported. These cells promote episomal persistence of plasmids carrying EBV ori $\mathrm{P}$ and can be used for large-scale transfection in suspension culture (Segura et al., 2007).

The first critical point in viral vector manufacturing is the condition of the cell culture. The 293T cells should not be passaged more than 18-20 times before transfection. In the classical method, cells cultured as monolayers in medium supplemented with $10 \%$ fetal bovine serum (FBS) should be subconfluent (50-80\% confluence) at the day of transfection (uniform distribution of plated cells is crucial). Production of lentiviral vectors by transient transfection of adherent cells growing in lower serum content or in serum-free media has also been achieved (Reiser, 2000; Geraerts et al., 2005; Mitta et al., 2005). The use of serum-free media helps to avoid the undesired in- fluence of serum-derived contaminants on target cell infection. Some recently reported simplified methods use cells growing in suspension and without serum (Segura et al., 2007; Kuroda et al., 2009; Ansorge et al., 2009) in order to reduce time, labor and cost of the procedure.

Another important issue with regard to transfection efficiency is the quality of plasmid DNA preparation. Plasmid DNA used for vector production must be free of bacteria-derived impurities (endotoxin free) and preferentially should be in a supercoiled form (scDNA). High yields of good quality DNA preparations are usually achieved using commercially available kits (Sigma-Aldrich, Promega, Invitrogen, Qiagen or equivalent plasmid DNA maxiprep kits). The total amount of DNA to be used for transfection (mixed plasmids) depends on the cell culture scale and usually ranges between 1 and $10 \mu \mathrm{g}$ per $10^{6}$ cells. In addition, a proper ratio of vector, helper(s) and envelope plasmid DNAs must be established. Typically, for the three-plasmid systems this ratio is 3:2:1, respectively (Kuroda et al., 2009; Kutner et al., 2009a; Kutner et al., 2009b), and for four plasmids - 2:1:1:1 (Mitta et al., 2005; Segura et al., 2007).

\section{Transient transfection}

The routine and most efficient method for cells transfection is based on DNA/calcium phosphate (CaP) coprecipitation using HEPES-buffered saline (HBS) or BES-buffered saline (BBS) solutions. This procedure can be carried out on a small scale in multiwell plates (micro- or milliliters of medium) as well as on a large scale in big culture 
vessels and bioreactors (liters of medium). Nonetheless, this procedure is labor-intensive and the reproducibility of results depends greatly on the $\mathrm{pH}$ of the buffer. Inconsistencies in $\mathrm{pH}$ may lead to significant batch-to-batch variations in titers. Therefore, samples of freshly prepared buffers that differ slightly in $\mathrm{pH}$ must be tested every time to get the most efficient transfection. Moreover, HBS is unstable and should be stored at $-20^{\circ} \mathrm{C}$. Additionally, the proper size of DNA/calcium phosphate crystals is of great importance for the transfection efficiency, which requires careful preparation of the mixture.

Hence, other methods have been used based on commercial lipid-based transfection reagents (FuGene, Roche; TransFectin, Bio-Rad; Lipofectamine, Invitrogen; etc). This technique offers good reproducibility, however, such reagents are more expensive than "homemade" solutions and are less efficient (Karolewski et al., 2003). Nonetheless, manufacturers still search for potent transfection methods and new promising products appear on the market. For example, a new generation of transfection reagents has recently been released by Clontech (Xfect). These are polymer-based biodegradable nanoparticles that are claimed to facilitate high transfection efficiency in a wide variety of mammalian cell types. Similarly, new polyaminebased transfection reagents (TransIT, Mirus Bio) outperform, as advertised by the manufacturer, lipid-based agents.

In order to develop a more reliable and cheaper method, several groups have tested transient poly(ethylene imine) (PEI)-based transfection (Geraerts et al., 2005; Segura et al., 2007; Kuroda et al., 2009; Ansorge et al., 2009; Bagnis et al., 2009). This method is ideally suited for large-scale vector production, including cell factories or bioreactors (Geraerts et al., 2005; Segura et al., 2007; Ansorge et al., 2009). PEI is relatively cheap and can be used in serum-free conditions. Moreover, PEI is chemically stable and ensures efficient transfection at a broad $\mathrm{pH}$ range. In direct comparison, the titers of vectors produced using PEI-mediated transfection are slightly lower than those obtained with the CaP-based method (Kuroda et al., 2009), but the PEI-based vector production is more cost-effective and the protocol is shorter.

To enhance transient transfection efficiency, many protocols include addition of chloroquine (4aminoquinoline) at the moment of DNA delivery (25 $\mu \mathrm{M}$ final concentration). This chemical is believed to reduce DNA degradation in lysosomes, but is toxic to the cells. It was shown that when used with PEI, chloroquine decreases viral titers when DMEM medium is used (Kuroda et al., 2009). Thus, addition of sodium butyrate instead of chloroquine might be beneficial in some experimental settings (Karolewski et al., 2003; Sena-Esteves et al., 2004; Ansorge et al., 2009).

The biochemical properties of the manufactured vector particles are different from those of the wild-type virus. For example, significantly fewer host cell proteins were found in lentiviral vector preparations than in HIV-1 virions (93 vs. 253) and the number as well as the composition of these proteins depends on the glycoprotein and transfer vector used (Wheeler et al., 2007; Denard et al., 2009). Moreover, only a small portion of the identified cellular proteins (25) was shared between HIV-1 and the lentiviral vector (Chertova et al., 2006; Wheeler et al., 2007). Nevertheless, as one might expect, the number of proteins present in vector and HIV preparations strongly depends on the purification conditions. Denard et al. (2009) estimated the number of possible virion-incorporated cellular proteins to as little as 23 while another 10 were found to co-purify with the vector. Among them, only 14 (or possibly even 9) were common for HIV-1 and lentiviral vectors, including syntenin-1, HSP70, HSC71, clathrin, actin, $\alpha$-enolase, GAPDH, eEF1A, and ALIX.

In summary, lentiviral vector particles are usually manufactured by the vector plasmids cotransfection of producer cells (preferentially the HEK 293 T cells). In order to achieve satisfactory viral titers all steps of the procedure must be tightly controlled. Additionally, the quality of DNA as well as proper cell culture status are crucial to get good results.

\section{CONCENTRATION OF LENTIVIRAL VECTORS PREPARATIONS}

Typical virus titers obtained from transient transfection range between $10^{6}$ and $10^{8} \mathrm{TU} / \mathrm{ml}$ of crude viral preparation and are sufficient for most in vitro studies based on transgene delivery. However, for gene silencing purposes or in vivo gene delivery, high multiplicities of infection (MOIs) in a limited volume are required. Thanks to the durability of pseudotyped viral vectors, increased titers can be achieved by physical concentration.

Notably, it is imperative that vector stocks to be used for clinical applications must be both concentrated and free of all impurities. Segura et al. (2006) published a detailed review on downstream processing procedures applied for retroviral vectors manufactured for gene therapy purposes.

The first clinical grade lentiviral vector, VRX496 (VIRxSYS Corporation), approved by the FDA's Cellular, Tissue, and Gene Therapies Advisory Committee (formerly called the Biological Response Modifiers Advisory Committee) for phase I pilot study, was manufactured by calcium phosphate 
cotransfection of 293 cells with vector and helper plasmids (a two-plasmid system relying on expression of VSV-G from packaging vector, VIRPAC) in a 10-layer cell factory system. Vector-containing cell culture medium was collected at several time points after transfection. The bulk-harvested vector was filtered and concentrated by hollow fiber ultrafiltration. Contaminating nucleic acids were eliminated by treatment with benzonase. The vector preparation was finally purified by size-exclusion chromatography, and sterile filtered through a $0.22-\mu \mathrm{m}$ filter (Slepushkin et al., 2003; Lu et al., 2004; Levine et al., 2006).

\section{Centrifugation}

The simplest and the most popular method for concentration of vector preparations is ultracentrifugation (Naldini et al., 1996a; Reiser, 2000; SenaEsteves et al., 2004; Kuroda et al., 2009; Kutner et al., 2009b). Virus-containing cell culture medium can be harvested two days after transfection or even one day later, if serum-free medium is used (Reiser, 2000; Geraerts et al., 2006), and then is filtered using a $0.45-\mu \mathrm{m}$ polysulfone filter. Viral particles are subsequently spun down for $1.5-2 \mathrm{~h}$ at $50-100.000 \times g$ and $4^{\circ} \mathrm{C}$. Resuspension of the pellet in a small volume of medium or buffer (e.g., phosphate-buffered saline, PBS) results in a more than hundredfold increase of the initial titer.

However, due to the shear forces generated during ultracentrifugation, this method is rather violent and may destroy certain vector pseudotypes. In such an event, centrifugation at a ten-fold reduced speed can be used to increase vector survival (Strang et al., 2004). Additionally, vectors carrying large inserts in their backbones (above $7 \mathrm{~kb}$ ) appeared to be particularly fragile and spinning at the reduced speed gave good virus recovery (al Yacoub et al., 2007). Alternatively, VSV-G-pseudotyped viral particles can be precipitated (e.g., using poly-L-lysine or PEG) prior to low-speed centrifugation (Zhang et al., 2001; Kutner et al., 2009b). These techniques are particularly useful for processing large volumes of vector stock (liters of vector-containing culture medium).

In large-scale vector production, reduction of media volume before centrifugation is desired. Geraerts et al. (2005) reported the use of tangential flow filtration (TFF) prior to centrifugation, which allowed 66-fold concentration of initial preparation. In the next step, centrifugation gave a further 30 -fold concentration.

Besides its roughness, ultracentrifugation may lead to problems with recovery of the vector. Pellets of viral particles produced in serum-free media are hard to resuspend and addition of $1 \mathrm{mM}$ EDTA or $8 \mu \mathrm{g} / \mathrm{ml}$ protamine sulfate to the resuspension buffer might be necessary (Kuroda et al., 2009). Another drawback of this method is that it concentrates all macromolecules present in the preparation (serum and host cell proteins, DNA), including inflammatory factors and potential viral infection inhibitors (reviewed by Segura et al., 2006). Therefore, to roughly purify vector stock, virus-containing culture medium can be ultracentrifuged through a sucrose gradient (Baekelandt et al., 2003) or cushion (Ricks et al., 2008; Kuroda et al., 2009).

\section{Ultrafiltration}

To bypass the problems outlined above, ultrafiltration can be used as an alternative (Reiser, 2000; Strang et al., 2004; Sena-Esteves et al., 2004; Segura et al., 2007; Miyake et al., 2007). For this purpose, Centricon Plus, Centriprep or Amicon 2000 and Amicon Ultra filters (all supplied by Millipore) with 50 or $100 \mathrm{kDa}$ molecular mass cut-off membranes can be applied. These devices were initially developed for protein concentration and desalting. However, using ultrafiltration membranes, vector concentration comparable to that offered by ultracentrifugation and even better recovery are obtained with a substantially lower centrifugation force $(2000-4000 \times g)$ and shorter spinning time (15-30 $\mathrm{min})$.

Additionally, this method enables removal of all contaminants smaller than the membrane pores. Indeed, Reiser (2000) observed vector titers much higher than expected from the achieved concentration factors and attributed this additional increase to the removal of putative transduction inhibitors.

\section{Chromatographic purification}

Unfortunately, ultrafiltration procedure also concentrates large host cell-derived contaminants which potentially could trigger immune response when vectors are used in vivo. Similarly to ultracentrifugation, another limitation of this method is its inability to handle large volumes of the preparation. Thus, for large-scale production of clinicalgrade Lentivirus-based vectors, a scalable purification and concentration technique is highly desirable. Chromatography-based methods seem to be ideally suited to these requirements. Vector preparations have been concentrated and purified using size-exclusion chromatography (Slepushkin et al., 2003), anion exchange chromatography (Yamada et al., 2003) and heparin affinity chromatography (Segura et al., 2007). However, all these methods give only about $50-70 \%$ recovery of the vector.

An interesting alternative method for simplified concentration and purification of lentiviral vectors by anion exchange chromatography is offered by Mustang Q Acrodiscs (Pall Corporation) (Marino et al., 2003; Slepushkin et al., 2003; Ricks et al., 2008; Kutner et al., 2009a; 2009b). The 
membranes used in these units are strong anion exchangers with faster flow rates than achievable by traditional ion exchange resins, which makes the pre-concentration step dispensable. Reported yields of vector recovery exceed $75 \%$ (Kutner et al., 2009a). Additionally, the Mustang Q Acrodiscs are designed for single use and can be adapted for both syringe bench-scale work and capsules for larger-volume applications (the capsule volumes range from 0.35 to $900 \mathrm{ml}$, enabling the processing of an estimated 1500 liters of vector medium per day). However, as for other chromatography resins, such membranes also bind serum- and cellderived proteins as well as contaminating nucleic acids from crude vector preparations, and efficient vector recovery requires that low amounts of the impurities be present in culture medium (Kutner et al., 2009a; 2009b). Moreover, it was also reported that vector stocks concentrated with this method are more sensitive to freeze/thawing (Kutner et al., 2009a). This might be explained by a low content of protein impurities in the final preparation, since HIV-1-derived vector particles pseudotyped with VSV-G, have been shown to be particularly sensitive to freezing when harvested in serum-free media (Strang et al., 2004).

\section{Stability of purified vector particles}

The sensitivity of vector stocks to freeze/thaw cycles is part of a larger problem - the thermolability of viral particles. In order to address this issue Strang et al. (2004) have tested the stability of different pseudotypes at $37^{\circ} \mathrm{C}$. Regardless of the presence of serum, VSV-G-containing particles were stable up to six hours of incubation, whereas HIV-1 vectors with gamma-retrovirus envelope glycoproteins were partially inactivated already after two hours. Other studies showed that serum-free conditions during vector production had a negative impact on the stability of purified vector stocks. For example, it was observed that for gamma-retrovirus envelope pseudotypes the rapid decrease in infectivity at $37^{\circ} \mathrm{C}$ was a consequence of the inactivation of the reverse transcription process (Carmo et al., 2009a). Since stability of vector particles produced for gene therapy applications is crucial, methods for protection of purified viral stocks are highly desired. It was recently demonstrated that addition of recombinant human serum albumin (rHSA) and lipoproteins to the storage buffer significantly increases stability of purified lentiviral vectors (Carmo et al., 2009b).

In summary, the concentration and purification of a crude vector stock is necessary for most in vivo applications. Particularly, for the clinical grade vectors a multistep procedure is required. The method of choice for such vector preparation are chromatography-based techniques.

\section{TITRATION OF LENTIVIRAL VECTOR STOCKS}

A variety of quantification methods for vector titer assessment are in use (for review see: Segura et al., 2006), each with specific advantages and disadvantages. Depending on particular needs, one titration method should be preferred over the others, taking into account that results can be either overor underestimated. Hence, a combination of methods can be used in order to obtain the most reliable results. Protocols for the most often used titration methods are described in Kutner et al. (2009b).

The vector titration methods can be divided into two groups: 1) assays for assessment of the total number of vector particles present, and 2) functional titers, which correspond to the concentration of vector particles capable of transducing target cells. Although functional titers have greater importance in most applications, the ratio between the number of infective particles and the total number of vectors gives an idea about the preparation quality and helps monitor vector production reproducibility. In this section we will discuss differences in titers obtained using distinct methods, pointing at possible sources of errors.

\section{Quantification of total number of vector particles}

The total number of virus particles can be measured directly in a vector preparation by detection of vector components:

- p24 CA by enzyme-linked immunosorbent assay (ELISA);

- vector gRNA concentration in viral supernatant by quantitative reverse transcriptase polymerase chain reaction (qRT-PCR);

- RT activity by product-enhanced reverse transcriptase assay.

These methods are relatively quick and may be conducted using commercially available kits (e.g., p24gag ELISA, Perkin-Elmer, and the RT assay, Roche). However, these techniques usually overestimate the functional vector titer. Quantification of p24 molecules does not discriminate between free $\mathrm{CA}$ and CA that originates from vector particles, including those that are non-functional. Moreover, titers obtained in immunoassay can be affected by the ratio of plasmids used for vector production, independently of vector specific infectivity (Logan et al., 2004b). The qRT-PCR-based titers do not distinguish defective particles that contain gRNA. In turn, the RT-assay measures only the RT activity which can be different for distinct vectors.

\section{Functional titers}

The functional titer is usually evaluated by transduction of target cells with a serial dilution of the vector stock and is defined as the number 
of transducing units per $\mathrm{ml}$ of virus stock (TU/ml). Quantification of the infective vector particles can be achieved by the following techniques:

- marker transgene expression assays;

- quantification of proviral DNA using quantitative PCR (qPCR);

- measurement of transgene mRNA levels by qRTPCR.

Titration methods based on marker gene expression involve detection of fluorescent proteins (by FACS or fluorescence microscopy), immunodetection of non-fluorescent transgene product (also employing flow cytometry and microscopy), enzymatic assays (e.g., $\beta$-galactosidase assay for lac $Z$ marker gene activity), antibiotic resistance of transduced cells (usually for selection genes that confer resistance to puromycin or G418/neomycin; in this case titer is defined as $\mathrm{CFU} / \mathrm{ml}$ ). These approaches are very useful when the assessment of vector infectibility is the goal. Yet, there are some disadvantages associated with the measurement of transgene expression. Transduction of target cells and synthesis of the marker protein takes several days, which makes the assay time-consuming. Quantification of infectious particles directly relies on transduction efficiency that is dependent on several factors, such as: time of cell exposure to the virus, vector sample volume, cell culture medium volume (rate of virus diffusion), etc (Zhang et al., 2004b). Hence, these methods tend to underestimate the number of transduction competent vector particles. Indeed, in a direct comparison to proviral DNA-based method, EGFP expression-based titer was ten times lower. Even larger differences can be found if a weak promoter is used to drive the marker gene expression (Sastry et al., 2002). In summary, although the use of some marker proteins (e.g., EGFP) may seem easy and straightforward, it requires careful consideration and proper planning of the experiment (see notes in Bagnis et al., 2009).

Titers based on proviral DNA copy number are obtained using real-time qPCR and primers/ probes targeting vector sequences present in most lentiviral vectors derived from HIV-1 (e.g., 5' portion of gag or LTR). This method shares some limitations with the previous approach (i.e., transduction efficiency-dependence), but is independent of the marker gene or the promoter used. Nevertheless, DNA copy quantification can be affected by the limitations of PCR (e.g., accuracy of the standard curve). More importantly, the number of provirus integrations does not always correlate with the transgene expression level, due to chromosomal position effects (for references see section on Chromatin Insulators) and, thus, this approach may overestimate functional titers.

Therefore, the most accurate and reliable method for vector titration could be measurement of proviral mRNA levels by real-time qRT-PCR. This technique is marker expression-independent, yet measures real levels of transgene activity. It is particularly useful in the light of the fact that transgenes themselves can affect functional titers due to transgene-associated toxicity in both producer cells and target cells (Lizée et al., 2003). Selection of the target mRNA sequence that is commonly used in Lentivirus-based vectors (e.g., WPRE) can simplify experimental design (Lizée et al., 2003; Geraerts et al., 2006).

Since choosing an optimal method for vector stock titration is a challenge, several research groups have conducted comparative studies on the accuracy of different approaches. As mentioned above, calculation of proviral DNA copy number is more accurate than tracking EGFP signal in a situation when the actual number of particles capable of transducing target cells is of interest (Sastry et al., 2002). On the other hand, calculation of gRNA directly in virus supernatant gives overestimated titers. This can be partially attributed to the presence of background plasmids in the vector preparation, yet has little impact on protocols that include a transduction procedure and time for transgene expression (Sastry et al., 2002). Although plasmid carryover is minimal during ex vivo transduction, it might be a problem for some titration methods and in vivo applications. Hence, to eliminate DNA contaminants vector preparations can be treated with benzonase (Sastry et al., 2004) or DNaseI (Pluta et al., 2005) prior to transduction.

In a comprehensive study, Geraerts et al. (2006) compared lentiviral vector titration methods that measure the amount of p24 (in pg/ml), gRNA (equivalents/ml), EGFP-based transducing units (TU/ml) and proviral mRNA equivalents. Not surprisingly, methods based on p24 concentration turned out to be the least reliable for the evaluation of functional vector particles. Still, this cheap and fast technique can be routinely used for quality control of virus production (TU per pg of p24). Similarly, gRNA content poorly correlates with functional titers and is rather dependent on the transfer vector features. In turn, fluorescence- and mRNA-based titers are very similar to each other and can be applied alternatively.

\section{Transduction of target cells}

Since the functional titer depends on transduction efficiency, constant infection conditions should be kept for vector stock testing. In this regard also selection of proper target cells is important. The HEK 293T, human osteosarcoma (HOS) (ATCC No. CRL1543) and HeLa (ATCC No. CCL-2) cell lines are widely used for this purpose. All these cells are permissive to transduction, yet titers obtained in HeLa are usually lower (Kutner et al., 2009a). Although the traditional reporter gene expression assay uses 
adherent cells, employment of suspension-growing target cells, 293E cell line, was also reported (Segura et al., 2007). Because the overnight cell attachment and trypsinization steps are skipped in this protocol, the time and complexity of the assay are reduced.

For routine vector titer evaluation, reproducibility of transduction is more important than its efficiency. However, for research applications, especially when cell types that are hard to infect are the subject, any trick that can boost transduction is highly desired. Generally, since vector particles must diffuse in the medium to reach the target cells and only the Brownian motion promotes the contacts between viral particles and target cells, the total volume of medium covering the cells should be minimal. Additionally, prolonged incubation with the virus or delivery of the vector at the same MOI in only half of the volume significantly elevates titers.

The majority of currently used protocols include addition of polybrene (hexadimethrine bromide) into medium during transduction (at a final concentration of $8 \mu \mathrm{g} / \mathrm{ml}$ ). This polycationic agent is believed to promote virus-cell interactions by decreasing putative repulsive forces. The increase of vector titer after polybrene treatment can be six-fold (O'Doherty et al., 2000). However, this positive effect is envelope-dependent and a comparative study of different pseudotypes showed that in the case of VSV-G the presence of polybrene did not substantially enhance the lentiviral vector titer (Strang et al., 2004).

Since the infection efficacy depends on the rate at which the vector can reach target cells, methods for sedimenting viral particles during transduction might be helpful. According to this assumption, low-speed centrifugation $(1200 \times g$ for $2 \mathrm{~h})$ of the cell culture plate following virus application greatly enhanced transduction (up to 45-fold) ( $\mathrm{O}^{\prime}$ Doherty et al., 2000). This technique, referred to as spinoculation, simply accelerates the binding of viruses to target cells. However, similarly to polybrene, spinoculation increases the infection efficiency in a pseudotypedependent manner (Strang et al., 2004). As it was shown, for VSV-G-enveloped particles centrifugation gave less pronounced results ( $\mathrm{O}^{\prime}$ Doherty et al., 2000; Strang et al., 2004). Nevertheless, as one might expect, usage of polybrene and spinoculation simultaneously had an additive effect (Strang et al., 2004).

An adsorption of viral vectors to cells can be also enhanced by nanoparticles. Hofmann et al. (2009) reported successful transduction of various cell types under nonpermissive conditions with lentiviral vector particles coupled to magnetic nanoparticles (MNPs). MNP-assisted infection, under such cumbersome conditions as the presence of hydrodynamic forces (shaking of the culture plate) and lowered temperature, was efficiently facilitated by the magnets placed under the plate. Interestingly, this technique also enabled targeting of vector/MNP complexes and positioning of transduced cells in desired organs and tissues when vectors and cells were administered systemically and then mice were exposed to a magnetic field.

For vector-based gene delivery to extremely sensitive targets, such as HSPCs, minimal ex vivo manipulation combined with high MOIs are required. Hence, protocols for infection of these cells employ conditions that do not alter their proliferation potential or ability to maintain multilineage differentiation. Lentiviral vectors have a greater potential than gamma-retrovirus-based vectors to transduce hematopoietic stem cells since efficient gene transfer with the former virus requires shorter time of incubation and little (or no) cytokine stimulation (De Palma et al., 2003). Recently, application of retronectin (TaKaRa Bio), a recombinant human fibronectin fragment $\mathrm{CH}-296$, for Lentivirus vector-based transduction has been reported (Kurre et al., 2006; Lee et al., 2009; Millington et al., 2009). Retronectin facilitates co-localization of the virus particles and target cells. Although enhancement of HSPCs infection in the presence of this peptide is rather poor (two-four-fold), immobilized retronectin exerts a positive effect on the behavior of hematopoietic stem cells in ex vivo culture (retronectin-coated culture plates). A further increase in gene delivery efficiency can be obtained by combination of retronectin and spinoculation (Millington et al., 2009). Interestingly, when mixed with the vector sample, retronectin also increases marker gene expression in bone marrow cells after direct bone marrow injection (Lee et al., 2009).

Since the HIV-1 Tat protein shares some functional domains with fibronectin, its influence on infection efficiency has been investigated recently (Nappi et al., 2009). Indeed, immobilized Tat, as well as Tat-derived peptides, bound viral particles and facilitated transduction with input doses that were otherwise insufficient to infect target cells. In contrast to the transduction process itself, binding of the virus to Tat appeared to be Env-independent. Another interesting observation is that, although Tat adsorbs viruses within a region responsible for its transactivation activity, Tat-mediated transduction enhancement does not require transactivation.

In summary, different vector titration methods have the specific strengths and weaknesses. Some of the methods tend to underestimate the number of infectious particles, other - to give overestimated results. Hence, the choice of the way to quantify vector particles depends on the particular needs and experimental setup. For example, FACS analysis of the cells expressing fluorescent marker can be used if transgene expression is of interest. On the other hand, measurement of proviral mRNA levels by real-time qRT-PCR is useful when the marker gene expression 
is not planned. Finally, since the methods estimating functional titers are based on the cell transduction, reproducibility of infection procedure is crucial.

\section{APPLICATIONS OF LENTIVIRAL VECTORS}

During the last decade, lentiviral vectors have emerged as popular gene delivery tools in many areas of basic and applied research. An ever increasing number of scientific publications provides new facts about its flexibility and versatility. Here, we will give a brief outline of the state of the art of Lentivirus-derived vector applications.

\section{APPLICATIONS OF LENTIVIRAL VECTORS FOR GENOME-WIDE FUNCTIONAL STUDIES OF GENE EXPRESSION}

The ability to study genes function on a genome-wide scale is of tremendous value since it enables rapid identification of the major regulators of biological pathways and, thus, provide a global view of mammalian genetic circuits.

Recently, a combination of Lentivirus-based vectors and RNAi technology has led to the establishment of libraries for high-throughput loss-offunction screens in a wide range of mammalian cell types, including primary and nondividing cells (Root et al., 2006). The reported libraries currently contain over 135000 lentiviral clones targeting 27000 human and mouse genes with multiple sequence-verified constructs. Using this technique, it was possible to identify more than one hundred putative regulators of mitotic progression and proliferation in human cancer cells (Moffat et al., 2006) or to find kinases, whose silencing modulates sensitivity of tumor cells to an inhibitor of the mitotic spindle protein kinesin- 5 (kinesin-5i) (Klinghoffer et al., 2008). In a similar approach, screening of the arrayed lentivirus library expressing shRNAs targeting over 5000 human druggable genes for silencing events that activate $\beta$-catenin pathway identified dihydrofolate reductase (DHFR) as a novel modulator of the $\beta$-catenin and GSK3 signaling (Klinghoffer et al., 2009). Reverse genetic screens showed their potential also in studies of the hematopoietic system. Using a lentiviral shRNA library, Ali et al. (2009) identified novel specific gene targets capable of altering HSPCs differentiation as well as shRNA constructs affecting stem cell expansion.

An interesting system for genome-wide studies of gene expression on the protein level has been described by Bialkowska et al. (2005). They presented genetic tool for protein tagging in mammalian cells based on lentiviral vector that harbors an artificial exon encoding EGFP tag. According to the preference of Lentivirus to integrate into transcriptionally active regions of host genome insertion of the artificial exon within introns of cellular genes resulted in expression of hybrid proteins consisting of the tag sequence fused in-frame to sequences of a cellular protein. Importantly, the tagged proteins were expressed from their endogenous promoters and the EGFP tag did not affect their subcellular localization. This technique enabled identification of tagged proteins by sequencing of RACE (rapid amplification of cDNAs ends) - PCR products and investigation of their subcellular distribution under variable physiological conditions using confocal microscopy. The protein tagging strategy could be also used for protein purification and studies on the protein-protein interactions.

\section{LENTIVIRAL VECTORS IN ANIMAL TRANSGENESIS}

The implementation of the Lentivirus-based gene delivery system in animal transgenesis has been discussed in detail by others (Park, 2007; Singer \& Verma, 2008; Pfeifer \& Hofmann, 2009). It is noteworthy that besides transgenic mice, rats, pigs, chickens and cows (see references in Park, 2007), also less popular species of transgenic cloned animals were generated recently using lentiviruses such as domestic cats (Gómez et al., 2009) and prairie voles (Donaldson et al., 2009). Genetic-modified animals are created by infection of fertilized or unfertilized oocytes, single-cell embryos, early blastocysts, embryonic stem cells or by transduction of cells that are used as donors of nucleus for somatic cell nuclear transfer (SCNT). Importantly, the progeny of these animals inherit and express the transgene.

Although lentiviral vectors have a smaller payload than is achievable with DNA-based methods, gene delivery via DNA microinjection displays a relatively low efficacy. Moreover, lentiviral-modified embryos show very high transgene expression levels and better survival rate. Utilization of Lentivirus-based vectors enables generation of transgenic animals with a tissue-specific and/or conditional expression of the desired gene or gene silencing. Therefore, this technique offers a good alternative for efficient and low-cost animal transgenesis for research applications, agriculture, biotechnology and biomedicine. It is particularly important in the light of the fact that large animals could be modified to become organ donors (pigs) or living protein factories (cows). Last but not least, the progress in Lentivirus-based transgenesis opens a gate to creation of virtually all possible animal models of human diseases. Recently, successful generation of the lentiviral-modified transgenic primate model of Huntington's disease has been reported (Yang et al., 2008d). Due to the close genetic, physiological and neuro- 
logical similarities between humans and monkeys, non-human primates are ideal models for studying human physiology and diseases.

\section{LENTIVIRAL VECTORS FOR CELL ENGINEERING}

Lentivirus-based vectors have recently emerged as the convenient tool for reprogramming of somatic cells. Induced pluripotent stem (iPS) cells focus recent attention as the possible source of autologous stem cells for use in regenerative medicine. Pluripotency can be induced in differentiated murine and human cells (e.g., fibroblasts) by lentiviral transduction of four transcription factors: Oct4, Sox2, Klf4, and c-Myc, as was originally reported by Takahashi \& Yamanaka (2006) who used gammaretroviral vector.

Brambrink et al. (2008) obtained iPS cells from mouse embryonic fibroblasts (MEFs) infected with four vectors expressing the transcription factors from Dox-inducible promoters. Interestingly, it was demonstrated that the generation of iPS cells required the ectopic expression of the transcription factors for a minimum of 12-16 days. Moreover, normal differentiation of already derived iPS cells required subsequent downregulation of the transgenes. Another group used similar Dox-inducible lentiviral system to generate human iPS cells from keratinocytes and found that these cells required only 10 days of expression of the reprogramming factors (Maherali et al., 2008). Switching Dox-inducible gene expression off and again on, enabled pluripotent cells differentiation and subsequent generation of "secondary" iPS cells at a frequency much greater than the initial conversion (Hockemeyer et al., 2008; Maherali et al., 2008).

However, the use of multiple vectors for cell reprogramming results in the high numbers of genomic integrations in iPS cells which limits their use for therapeutic applications. Hence, several research groups (e.g., Sommer et al., 2009; Carey et al., 2009; Shao et al., 2009) have independently demonstrated generation of iPS cells with single lentiviral vector expressing a "stem cell cassette" composed of all four transcription factors. This powerful, yet simple system, expressing four genes from a single transcript (using 2A peptide sequences or a combination of 2A peptide and IRES), allowed induction of pluripotency with a single viral integration. The next step toward the application of iPS technology for clinical purposes was the implementation of the Cre/loxP recombination system. After induction of pluripotency in adult skin fibroblast by their transduction with single polycistronic lentiviral vector, expression of the Cre recombinase in generated iPS cells resulted in deletion of the vector from the host DNA (Chang et al., 2009).
Such lentiviral vector particles that induce pluripotency of transduced cells are commercially available (Allele Biotechnology).

\section{LENTIVIRAL VECTORS IN CLINICAL GENE THERAPY APPLICATIONS}

Potential gene therapy targets accessible to lentiviral vectors but out of reach for most other gene delivery agents can be found in the CNS, liver, heart, kidney, muscle, ocular tissue and pancreas. Successful preclinical studies have been carried out for treatment of Alzheimer's, Parkinson's and Huntington's diseases. Unlike the CNS or other organs, therapy of most genetic disorders linked to the hematopoietic system does not require in vivo administration of the vector and can be achieved by an ex vivo transduction and subsequent transplantation of isolated autologous HSPCs. Over the last decade, several research groups have reported successful correction of genetic disorders, including immunodeficiencies and hemoglobinopathies, in preclinical animal models (for references see: Wiznerowicz \& Trono, 2005; Ralph et al., 2006; Cockrell \& Kafri, 2007; Neschadim et al., 2007; Nanou \& Azzouz, 2009).

The first clinical study using a lentiviral vector was approved in 2001 for anti-HIV therapy (MacGregor, 2001). This trial was performed to evaluate the safety and efficiency of a conditionally replicating HIV-1-derived vector delivering HIV-1 envelope antisense gene to $\mathrm{CD} 4+\mathrm{T}$ cells. Of note, the design of the vector (transfer vector contains both LTRs) makes expression of the env antisense RNA Tat- and Rev-dependent and, thus, basal expression increases when wild-type HIV infects vector-containing cells. In four of five patients with chronic HIV infection enrolled in this study, immune function improved after infusion of $10^{10}$ ex vivo gene-modified autologous CD4+ $\mathrm{T}$ cells. The presented results showed an absence of RCLs and a lack of clonal outgrowths (potential precursors to insertional mutagenesis) after 21-36 months of observation (Levine et al., 2006). Additionally, a high-throughput analysis of vector integration sites in ex vivo vector-transduced CD4+ T cells and in cells recovered at several time points after infusion clearly showed preference for active genes and epigenetic marks associated with active transcription units. There was also no indication that proliferation of the transduced cells after infusion resulted in any enrichment for integration sites in proximity of protooncogenes or within tumor suppressor genes (Wang et al., 2009a). Altogether, these reports show the clinical usefulness of the $\mathrm{T}$ cell culture system and gene transfer using lentiviral vectors.

Since that time, twenty-one clinical trials with Lentivirus-based vectors have been initiated or are 
currently under review (including eight new clinical trials opened in 2008 and two under revision in 2009). This number constitutes $1.4 \%$ of the total 1537 gene therapy clinical trials employing distinct gene delivery methods. To date (March 2009), all trials, including phase I/II studies, were based on ex vivo vector delivery. Eight of the trials were performed for the treatment of HIV infection, nine for treatment of genetic diseases (X-linked cerebral adrenoleukodystrophy, sickle cell anemia, $\beta$-thalassemia, Wiskott-Aldrich syndrome, mucopolysaccharidosis type VII, Fanconi anemia complementation group A, Parkinson's disease, X-SCID) and four trials were directed against various cancers (data provided by J Gene Med; see List of websites [5]).

There is also a growing interest in the development of lentiviral vector-based vaccines against various diseases, including HIV infection (reviewed by Lemiale \& Korokhov, 2009). Just recently, nonintegrating Lentivirus-based vectors have been shown to confer in mice immunity against West Nile virus (WNV) (Coutant et al., 2008), HBV (Karwacz et al., 2009) and against tumor cells expressing the ovalbumin (OVA) antigen (Hu et al., 2009b; Karwacz et al., 2009). Another interesting application of vectors derived from Lentivirus is in wound healing. Badillo et al. (2007) demonstrated in an animal model that lentiviral-mediated overproduction of stromal-derived growth factor- $1 \alpha$ (SDF-1 $\alpha$ ) is sufficient to correct the pathophysiologic abnormalities associated with diabetic wound healing.

\section{CONCLUDING REMARKS}

Molecular studies that were initiated more than two decades ago, have led to the development of efficient and safe gene transfer system that is being successfully used for a wide variety of sophisticated in vitro and in vivo experiments. This was made possible by the constantly expanding knowledge about the virus structure and biology that we have gained from the decades-lasting research. However, this knowledge is far from being complete.

Vectors that utilize lentiviral genomes are characterized by a broad range of targets, long-lasting expression, relatively high capacity and low toxicity. They are also easy to adopt to such interesting and potent techniques as RNAi technology and targeted regulatable gene expression. Vectors derived from lentiviruses have also demonstrated their great potential in the generation of transgenic animals expected to be extremely beneficial in modern agriculture, biotechnology and biomedicine.

Lentivirus-based methods of gene delivery have proven their indisputable superiority over simple retroviruses in safe modification of quiescent, non-stimulated hematopoietic progenitor cells $e x$ vivo, potent delivery of genetic payload to neurons in the CNS and to other differentiated cells in distinct organs (liver, pancreas, etc). They also appeared to be more potent than non-viral gene delivery methods in different experimental settings (Dullaers et al., 2004; Cao et al., 2009; Kim et al., 2009). Consequently, lentiviral vectors have been successfully used for treatment of various genetic deficiencies and physiological disorders in animal models. Results from preclinical studies have provided grounds for the application of these vectors in human gene therapy.

The stigma understandably associated with HIV-1-based vectors forced the tremendous progress in vector system development in terms of its safety and availability of a variety of standardized procedures and assays for vector evaluation. That led to the first clinical trial involving a lentiviral genomebased vector to be approved eight years ago. Although, to the best of our knowledge, a satisfactory result of a clinical trial is yet to be reported, with the search for a perfect vector ongoing, such a breakthrough report cannot be far.

\section{Acknowledgements}

The authors express their deepest appreciations to Dr. J. Reiser and Dr. D. Pijanowska for the fruitful discussion and their help in preparation of the manuscript.

We also apologize to those investigators whose studies could not be presented here due to space limitations.

\section{LIST OF WEBSITES}

1. www.emea.europa.eu

2. www.fda.gov/ForConsumers/ByAudience/ForPatientAdvocates/HIVandAIDSActivities/ucm118915.htm

3. www.ncbi.nlm.nih.gov/ICTVdb/ICTVdB/00.061.1.06. htm

4. www.ncbi.nlm.nih.gov/Taxonomy/Browser/wwwtax.cgi? $i d=11632 \&$ keep $=1 \& l v l=6 \&$ filter=genome filter\&p=genome

5. www.wiley.co.uk/genmed/clinical/

6. www.unaids.org/en/KnowledgeCentre/HIVData/GlobalReport/2008/2008_Global_report.asp

7. www.hiv.lanl.gov/content/sequence/HIV/COMPENDI$\mathrm{UM} / 2009 /$ sequence2009.pdf

8. www.ncbi.nlm.nih.gov/RefSeq/HIVInteractions/

9. www.emea.europa.eu/pdfs/human/bwp/245803en.pdf

10. oba.od.nih.gov/oba/rac/Guidance/LentiVirus_Containment/pdf/Lenti_Containment_Guidance.pdf

11. www.jove.com/index/Details.stp? ID $=550$

\section{REFERENCES}

Abrahamyan LG, Markosyan RM, Moore JP, Cohen FS, Melikyan GB (2003) Human immunodeficiency virus 
type 1 Env with an intersubunit disulfide bond engages coreceptors but requires bond reduction after engagement to induce fusion. J Virol 77: 5829-5836.

Adamson CS, Freed EO (2007) Human immunodeficiency virus type 1 assembly, release, and maturation. Adv Pharmacol 55: 347-387.

Agha-Mohammadi S, Lotze MT (2000) Regulatable systems: applications in gene therapy and replicating viruses. J Clin Invest 105: 1177-1183.

Agha-Mohammadi S, O'Malley M, Etemad A, Wang Z, Xiao X, Lotze MT (2004) Second-generation tetracycline-regulatable promoter: repositioned tet operator elements optimize transactivator synergy while shorter minimal promoter offers tight basal leakiness. J Gene Med 6: 817-828.

Aguiar RS, Peterlin BM (2008) APOBEC3 proteins and reverse transcription. Virus Res 134: 74-85.

Aiken C (1997) Pseudotyping human immunodeficiency virus type 1 (HIV-1) by the glycoprotein of vesicular stomatitis virus targets HIV-1 entry to an endocytic pathway and suppresses both the requirement for Nef and the sensitivity to cyclosporin A. J Virol 71: 58715877.

Aker M, Tubb J, Groth AC, Bukovsky AA, Bell AC, Felsenfeld G, Kiem HP, Stamatoyannopoulos G, Emery DW (2007) Extended core sequences from the cHS4 insulator are necessary for protecting retroviral vectors from silencing position effects. Hum Gene Ther 18: 333-343.

Akira S, Uematsu S, Takeuchi O (2006) Pathogen recognition and innate immunity. Cell 124: 783-801.

Akkina RK, Walton RM, Chen ML, Li QX, Planelles V, Chen IS (1996) High-efficiency gene transfer into CD34+ cells with a human immunodeficiency virus type 1-based retroviral vector pseudotyped with vesicular stomatitis virus envelope glycoprotein G. J Virol 70: 2581-2585.

al Yacoub N, Romanowska M, Haritonova N, Foerster J (2007) Optimized production and concentration of lentiviral vectors containing large inserts. J Gene Med 9: 579-584.

Ali N, Karlsson C, Aspling M, Hu G, Hacohen N, Scadden DT, Larsson J (2009) Forward RNAi screens in primary human hematopoietic stem/progenitor cells. Blood 113: 3690-3695.

Alkhatib G, Combadiere C, Broder CC, Feng Y, Kennedy PE, Murphy PM, Berger EA (1996) CC CKR5: a RANTES, MIP-1alpha, MIP-1beta receptor as a fusion cofactor for macrophage-tropic HIV-1. Science 272: 1955-1958.

Amanna IJ, Slifka MK (2009) Wanted, dead or alive: New viral vaccines. Antiviral Res 84: 119-130.

Amar L, Desclaux M, Faucon-Biguet N, Mallet J, Vogel R (2006) Control of small inhibitory RNA levels and RNA interference by doxycycline induced activation of a minimal RNA polymerase III promoter. Nucleic Acids Res 34: e37.

Amendola M, Venneri MA, Biffi A, Vigna E, Naldini L (2005) Coordinate dual-gene transgenesis by lentiviral vectors carrying synthetic bidirectional promoters. Nat Biotechnol 23: 108-116.

Amini S, Khalili K, Sawaya BE (2004) Effect of HIV-1 Vpr on cell cycle regulators. DNA Cell Biol 23: 249-260.

Andersen JL, Le Rouzic E, Planelles V (2008) HIV-1 Vpr: mechanisms of G2 arrest and apoptosis. Exp Mol Pathol 85: 2-10.

Anderson EC, Lever AM (2006) Human immunodeficiency virus type $1 \mathrm{Gag}$ polyprotein modulates its own translation. J Virol 80: 10478-10486.
Anderson JL, Hope TJ (2005) Intracellular trafficking of retroviral vectors: obstacles and advances. Gene Ther 12: 1667-1678.

Anderson JL, Hope TJ (2008) APOBEC3G restricts early HIV-1 replication in the cytoplasm of target cells. Virology 375: 1-12.

Anderson JL, Johnson AT, Howard JL, Purcell DF (2007) Both linear and discontinuous ribosome scanning are used for translation initiation from bicistronic human immunodeficiency virus type 1 env mRNAs. J Virol 81: 4664-4676.

Ansorge S, Lanthier S, Transfiguracion J, Durocher Y, Henry O, Kamen A (2009) Development of a scalable process for high-yield lentiviral vector production by transient transfection of HEK293 suspension cultures. J Gene Med 11: 868-876.

Ao Z, Yao X, Cohen EA (2004) Assessment of the role of the central DNA flap in human immunodeficiency virus type 1 replication by using a single-cycle replication system. J Virol 78: 3170-3177.

Ao Z, Huang G, Yao H, Xu Z, Labine M, Cochrane AW, Yao X (2007) Interaction of human immunodeficiency virus type 1 integrase with cellular nuclear import receptor importin 7 and its impact on viral replication. $J$ Biol Chem 282: 13456-13467.

Ao Z, Yu Z, Wang L, Zheng Y, Yao X (2008) Vpr14-88Apobec3G fusion protein is efficiently incorporated into Vif-positive HIV-1 particles and inhibits viral infection. PLoS ONE 3: e1995.

Apolonia L, Waddington SN, Fernandes C, Ward NJ, Bouma G, Blundell MP, Thrasher AJ, Collins MK, Philpott NJ (2007) Stable gene transfer to muscle using non-integrating lentiviral vectors. Mol Ther 15: 1947-1954.

Arhel N, Genovesio A, Kim KA, Miko S, Perret E, OlivoMarin JC, Shorte S, Charneau P (2006) Quantitative four-dimensional tracking of cytoplasmic and nuclear HIV-1 complexes. Nat Methods 3: 817-824.

Arhel NJ, Souquere-Besse S, Munier S, Souque $P$, Guadagnini S, Rutherford S, Prevost MC, Allen TD, Charneau P (2007) HIV-1 DNA Flap formation promotes uncoating of the pre-integration complex at the nuclear pore. EMBO J 26: 3025-3037.

Arumugam PI, Scholes J, Perelman N, Xia P, Yee JK, Malik $P$ (2007) Improved human beta-globin expression from self-inactivating lentiviral vectors carrying the chicken hypersensitive site-4 (cHS4) insulator element. Mol Ther 15: 1863-1871.

Arumugam PI, Urbinati F, Velu CS, Higashimoto T, Grimes HL, Malik P (2009) The 3' region of the chicken hypersensitive site- 4 insulator has properties similar to its core and is required for full insulator activity. PLoS One 4: e6995.

Asang C, Hauber I, Schaal H (2008) Insights into the selective activation of alternatively used splice acceptors by the human immunodeficiency virus type-1 bidirectional splicing enhancer. Nucleic Acids Res 36: 1450-1463.

Auewarakul P, Wacharapornin P, Srichatrapimuk S, Chutipongtanate S, Puthavathana P (2005) Uncoating of HIV-1 requires cellular activation. Virology 337: 93-101.

Badillo AT, Chung S, Zhang L, Zoltick P, Liechty KW (2007) Lentiviral gene transfer of SDF-1alpha to wounds improves diabetic wound healing. J Surg Res 143: $35-42$.

Baekelandt V, Claeys A, Eggermont K, Lauwers E, De Strooper B, Nuttin B, Debyser Z (2002) Characterization of lentiviral vector-mediated gene transfer in adult mouse brain. Hum Gene Ther 13: 841-853.

Baekelandt V, Eggermont K, Michiels M, Nuttin B, Debyser Z (2003) Optimized lentiviral vector production and 
purification procedure prevents immune response after transduction of mouse brain. Gene Ther 10: 1933-1940.

Bagnis C, Bailly P, Chapel-Fernandes S (2009) Using an EGFPmeter to evaluate the lentiviral vector production: tricks and traps. Methods Mol Biol 515: 151-163.

Barat C, Lullien V, Schatz O, Keith G, Nugeyre MT, Gruninger-Leitch F, Barre-Sinoussi F, LeGrice SF, Darlix JL (1989) HIV-1 reverse transcriptase specifically interacts with the anticodon domain of its cognate primer tRNA. EMBO J 8: 3279-3285.

Barboric M, Peterlin BM (2005) A new paradigm in eukaryotic biology: HIV Tat and the control of transcriptional elongation. PLoS Biol 3: e76.

Baron U, Bujard H (2000) Tet repressor-based system for regulated gene expression in eukaryotic cells: principles and advances. Methods Enzymol 327: 401-421.

Barraud P, Paillart JC, Marquet R, Tisne C (2008) Advances in the structural understanding of Vif proteins. Curr HIV Res 6: 91-99.

Barre-Sinoussi F, Chermann JC, Rey F, Nugeyre MT, Chamaret S, Gruest J, Dauguet C, Axler-Blin C, Vezinet-Brun F, Rouzioux C, Rozenbaum W, Montagnier L (1983) Isolation of a T-lymphotropic retrovirus from a patient at risk for acquired immune deficiency syndrome (AIDS) Science 220: 868-871.

Basu VP, Song M, Gao L, Rigby ST, Hanson MN, Bambara RA (2008) Strand transfer events during HIV-1 reverse transcription. Virus Res 134: 19-38.

Bayer M, Kantor B, Cockrell A, Ma H, Zeithaml B, Li X, McCown T, Kafri T (2008) A large U3 deletion causes increased in vivo expression from a nonintegrating lentiviral vector. Mol Ther 16: 1968-1976.

Ben-Dor I, Itsykson P, Goldenberg D, Galun E, Reubinoff BE (2006) Lentiviral vectors harboring a dual-gene system allow high and homogeneous transgene expression in selected polyclonal human embryonic stem cells. Mol Ther 14: 255-267.

Berkowitz R, Ilves H, Lin WY, Eckert K, Coward A, Tamaki S, Veres G, Plavec I (2001a) Construction and molecular analysis of gene transfer systems derived from bovine immunodeficiency virus. J Virol 75: 3371-3382.

Berkowitz RD, Ilves H, Plavec I, Veres G (2001b) Gene transfer systems derived from Visna virus: analysis of virus production and infectivity. Virology 279: 116-129.

Bhatia AK, Kaushik R, Campbell NA, Pontow SE, Ratner L (2009) Mutation of critical serine residues in HIV-1 matrix result in an envelope incorporation defect which can be rescued by truncation of the gp41 cytoplasmic tail. Virology 384: 233-241.

Bhattacharya J, Repik A, Clapham PR (2006) Gag regulates association of human immunodeficiency virus type 1 envelope with detergent-resistant membranes. J Virol 80: 5292-5300.

Bialkowska A, Zhang XY, Reiser J (2005) Improved tagging strategy for protein identification in mammalian cells. BMC Genomics 6: 113 .

Bieniasz PD (2006) Late budding domains and host proteins in enveloped virus release. Virology 344: 55-63.

Bilbille Y, Vendeix FA, Guenther R, Malkiewicz A, Ariza X, Vilarrasa J, Agris PF (2009) The structure of the human tRNALys3 anticodon bound to the HIV genome is stabilized by modified nucleosides and adjacent mismatch base pairs. Nucleic Acids Res 37: 3342-3353.

Bishop KN, Verma M, Kim EY, Wolinsky SM, Malim MH (2008) APOBEC3G inhibits elongation of HIV-1 reverse transcripts. PLoS Pathog 4: e1000231.

Blomer U, Naldini L, Kafri T, Trono D, Verma IM, Gage FH (1997) Highly efficient and sustained gene transfer in adult neurons with a lentivirus vector. I Virol 71: 6641-6649.

Board of the European Society of Gene, Cell Therapy (2008) Case of leukaemia associated with X-linked severe combined immunodeficiency gene therapy trial in London. Hum Gene Ther 19: 3-4.

Boasso A, Shearer GM (2008) Chronic innate immune activation as a cause of HIV-1 immunopathogenesis. Clin Immunol 126: 235-242.

Boasso A, Herbeuval JP, Hardy AW, Anderson SA, Dolan MJ, Fuchs D, Shearer GM (2007) HIV inhibits CD4+ T-cell proliferation by inducing indoleamine 2,3-dioxygenase in plasmacytoid dendritic cells. Blood 109: 3351-3359.

Boasso A, Hardy AW, Anderson SA, Dolan MJ, Shearer GM (2008) HIV-induced type I interferon and tryptophan catabolism drive $\mathrm{T}$ cell dysfunction despite phenotypic activation. PLOS ONE 3: e2961.

Boden D, Markowitz M (1998) Resistance to human immunodeficiency virus type 1 protease inhibitors. Antimicrob Agents Chemother 42: 2775-2783.

Bokhoven M, Stephen SL, Knight S, Gevers EF, Robinson IC, Takeuchi Y, Collins MK (2009) Insertional gene activation by lentiviral and gammaretroviral vectors. $J$ Virol 83: 283-294.

Bolinger C, Boris-Lawrie K (2009) Mechanisms employed by retroviruses to exploit host factors for translational control of a complicated proteome. Retrovirology 6: 8 .

Bowman MC, Archin NM, Margolis DM (2009) Pharmaceutical approaches to eradication of persistent HIV infection. Expert Rev Mol Med 11: e6.

Brady J, Kashanchi F (2005) Tat gets the "green" light on transcription initiation. Retrovirology 2: 69.

Brambrink T, Foreman R, Welstead GG, Lengner CJ, Wernig M, Suh H, Jaenisch R (2008) Sequential expression of pluripotency markers during direct reprogramming of mouse somatic cells. Cell Stem Cell 2: 151-159.

Brasey A, Lopez-Lastra M, Ohlmann T, Beerens N, Berkhout B, Darlix JL, Sonenberg N (2003) The leader of human immunodeficiency virus type 1 genomic RNA harbors an internal ribosome entry segment that is active during the G2/M phase of the cell cycle. $J \mathrm{Vi}$ rol 77: 3939-3949.

Brass AL, Dykxhoorn DM, Benita Y, Yan N, Engelman A, Xavier RJ, Lieberman J, Elledge SJ (2008) Identification of host proteins required for HIV infection through a functional genomic screen. Science 319: 921-926.

Bray M, Prasad S, Dubay JW, Hunter E, Jeang KT, Rekosh D, Hammarskjold ML (1994) A small element from the Mason-Pfizer monkey virus genome makes human immunodeficiency virus type 1 expression and replication Rev-independent. Proc Natl Acad Sci USA 91: 1256-1260.

Breckpot K, Dullaers M, Bonehill A, van Meirvenne S, Heirman $C$, de Greef C, van der Bruggen P, Thielemans K (2003) Lentivirally transduced dendritic cells as a tool for cancer immunotherapy. J Gene Med 5: 654-667.

Broussau S, Jabbour N, Lachapelle G, Durocher Y, Tom R, Transfiguracion J, Gilbert R, Massie B (2008) Inducible packaging cells for large-scale production of lentiviral vectors in serum-free suspension culture. Mol Ther 16: 500-507.

Brown BD, Sitia G, Annoni A, Hauben E, Sergi LS, Zingale A, Roncarolo MG, Guidotti LG, Naldini L (2007a) In vivo administration of lentiviral vectors triggers a type I interferon response that restricts hepatocyte gene transfer and promotes vector clearance. Blood 109: 2797-2805. 
Brown BD, Cantore A, Annoni A, Sergi LS, Lombardo A, Della Valle P, D’Angelo A, Naldini L (2007b) A microRNA-regulated lentiviral vector mediates stable correction of hemophilia B mice. Blood 110: 4144-4152.

Buchschacher GL Jr (2001) Introduction to retroviruses and retroviral vectors. Somat Cell Mol Genet 26: 1-11.

Buck CB, Shen X, Egan MA, Pierson TC, Walker CM, Siliciano RF (2001) The human immunodeficiency virus type 1 gag gene encodes an internal ribosome entry site. J Virol 75: 181-191.

Bukovsky AA, Song JP, Naldini L (1999) Interaction of human immunodeficiency virus-derived vectors with wild-type virus in transduced cells. J Virol 73: 70877092.

Bukrinskaya A (2007) HIV-1 matrix protein: a mysterious regulator of the viral life cycle. Virus Res 124: 1-11.

Bukrinskaya A, Brichacek B, Mann A, Stevenson M (1998) Establishment of a functional human immunodeficiency virus type 1 (HIV-1) reverse transcription complex involves the cytoskeleton. J Exp Med 188: 2113-2125.

Bukrinsky M (2004) A hard way to the nucleus. Mol Med 10: 1-5.

Bukrinsky MI, Stanwick TL, Dempsey MP, Stevenson M (1991) Quiescent T lymphocytes as an inducible virus reservoir in HIV-1 infection. Science 254: 423-427.

Bukrinsky MI, Sharova N, Dempsey MP, Stanwick TL, Bukrinskaya AG, Haggerty S, Stevenson M (1992) Active nuclear import of human immunodeficiency virus type 1 preintegration complexes. Proc Natl Acad Sci USA 89: 6580-6584.

Bukrinsky MI, Sharova N, McDonald TL, Pushkarskaya T, Tarpley WG, Stevenson M (1993a) Association of integrase, matrix, and reverse transcriptase antigens of human immunodeficiency virus type 1 with viral nucleic acids following acute infection. Proc Natl Acad Sci USA 90: 6125-6129.

Bukrinsky MI, Haggerty S, Dempsey MP, Sharova N, Adzhubel A, Spitz L, Lewis P, Goldfarb D, Emerman M, Stevenson M (1993b) A nuclear localization signal within HIV-1 matrix protein that governs infection of non-dividing cells. Nature 365: 666-669.

Burns JC, Friedmann T, Driever W, Burrascano M, Yee JK (1993) Vesicular stomatitis virus G glycoprotein pseudotyped retroviral vectors: concentration to very high titer and efficient gene transfer into mammalian and nonmammalian cells. Proc Natl Acad Sci USA 90: 80338037.

Bushman FD, Fujiwara T, Craigie R (1990) Retroviral DNA integration directed by HIV integration protein in vitro. Science 249: 1555-1558.

Busschots K, Vercammen J, Emiliani S, Benarous R, Engelborghs Y, Christ F, Debyser Z (2005) The interaction of LEDGF/p75 with integrase is lentivirus-specific and promotes DNA binding. J Biol Chem 280: 17841-17847.

Busschots K, Voet A, De Maeyer M, Rain JC, Emiliani S, Benarous R, Desender L, Debyser Z, Christ F (2007) Identification of the LEDGF/p75 binding site in HIV-1 integrase. J Mol Biol 365: 1480-1492.

Butsch M, Boris-Lawrie K (2000) Translation is not required to generate virion precursor RNA in human immunodeficiency virus type 1-infected T cells. J Virol 74: 11531-11537.

Campbell EM, Nunez R, Hope TJ (2004) Disruption of the actin cytoskeleton can complement the ability of Nef to enhance human immunodeficiency virus type 1 infectivity. J Virol 78: 5745-5755.

Cao F, Xie X, Gollan T, Zhao L, Narsinh K, Lee RJ, Wu JC (2009) Comparison of Gene-Transfer Efficiency in
Human Embryonic Stem Cells. Mol Imaging Biol (Epub ahead of print).

Carey BW, Markoulaki S, Hanna J, Saha K, Gao Q, Mitalipova M, Jaenisch R (2009) Reprogramming of murine and human somatic cells using a single polycistronic vector. Proc Natl Acad Sci USA 106: 157-162.

Carlton JG, Martin-Serrano J (2009) The ESCRT machinery: new functions in viral and cellular biology. Biochem Soc Trans 37: 195-199.

Carmo M, Dias JD, Panet A, Coroadinha AS, Carrondo MJ, Alves PM, Cruz PE (2009a) Thermosensitivity of the reverse transcription process as an inactivation mechanism of lentiviral vectors. Hum Gene Ther 20: 1168-1176.

Carmo M, Alves A, Rodrigues AF, Coroadinha AS, Carrondo MJ, Alves PM, Cruz PE (2009b) Stabilization of gammaretroviral and lentiviral vectors: from production to gene transfer. J Gene Med 11: 670-678.

Carneiro FA, Lapido-Loureiro PA, Cordo SM, Stauffer F, Weissmuller G, Bianconi ML, Juliano MA, Juliano L, Bisch PM, Da Poian AT (2006) Probing the interaction between vesicular stomatitis virus and phosphatidylserine. Eur Biophys J 35: 145-154.

CDC (1981) Pneumocystis pneumonia - Los Angeles. MMWR Morb Mortal Wkly Rep 30: 250-252.

Chang AH, Stephan MT, Sadelain M (2006) Stem cell-derived erythroid cells mediate long-term systemic protein delivery. Nat Biotechnol 24: 1017-1021.

Chang LJ, McNulty E, Martin M (1993) Human immunodeficiency viruses containing heterologous enhancer/ promoters are replication competent and exhibit different lymphocyte tropisms. J Virol 67: 743-752.

Chang LJ, Zaiss AK (2003) Self-inactivating lentiviral vectors and a sensitive Cre-loxP reporter system. Methods Mol Med 76: 367-382.

Chang MI, Panorchan P, Dobrowsky TM, Tseng Y, Wirtz D (2005) Single-molecule analysis of human immunodeficiency virus type 1 gp120-receptor interactions in living cells. J Virol 79: 14748-14755.

Charneau P, Alizon M, Clavel F (1992) A second origin of DNA plus-strand synthesis is required for optimal human immunodeficiency virus replication. J Virol 66: 2814-2820.

Chang CW, Lai YS, Pawlik KM, Liu K, Sun CW, Li C, Schoeb TR, Townes TM (2009) Polycistronic lentiviral vector for "hit and run" reprogramming of adult skin fibroblasts to induced pluripotent stem cells. Stem Cells 27: 1042-1049.

Charrier S, Dupre L, Scaramuzza S, Jeanson-Leh L, Blundell MP, Danos O, Cattaneo F, Aiuti A, Eckenberg R, Thrasher AJ, Roncarolo MG, Galy A (2007) Lentiviral vectors targeting WASp expression to hematopoietic cells, efficiently transduce and correct cells from WAS patients. Gene Ther 14: 415-428.

Chazal N, Singer G, Aiken C, Hammarskjold ML, Rekosh D (2001) Human immunodeficiency virus type 1 particles pseudotyped with envelope proteins that fuse at low $\mathrm{pH}$ no longer require Nef for optimal infectivity. $J$ Virol 75: 4014-4018.

Chen BJ, Lamb RA (2008) Mechanisms for enveloped virus budding: can some viruses do without an ESCRT? Virology 372: 221-232.

Chen ST, Iida A, Guo L, Friedmann T, Yee JK (1996) Generation of packaging cell lines for pseudotyped retroviral vectors of the $G$ protein of vesicular stomatitis virus by using a modified tetracycline inducible system. Proc Natl Acad Sci USA 93: 10057-10062.

Chen Y, Stamatoyannopoulos G, Song CZ (2003) Downregulation of CXCR4 by inducible small interfering 
RNA inhibits breast cancer cell invasion in vitro. Cancer Res 63: 4801-4804.

Cheng SM, Li JC, Lin SS, Lee DC, Liu L, Chen Z, Lau AS (2009) HIV-1 trans-activator protein induction of suppressor of cytokine signaling-2 contributes to dysregulation of IFN \{gamma\} signaling. Blood 113: 5192-5201.

Cherepanov P (2007) LEDGF/p75 interacts with divergent lentiviral integrases and modulates their enzymatic activity in vitro. Nucleic Acids Res 35: 113-124.

Cherepanov P, Devroe E, Silver PA, Engelman A (2004) Identification of an evolutionarily conserved domain in human lens epithelium-derived growth factor/transcriptional co-activator p75 (LEDGF/p75) that binds HIV-1 integrase. J Biol Chem 279: 48883-48892.

Cherepanov P, Maertens G, Proost P, Devreese B, Van Beeumen J, Engelborghs Y, De Clercq E, Debyser Z (2003) HIV-1 integrase forms stable tetramers and associates with LEDGF/p75 protein in human cells. J Biol Chem 278: 372-381.

Chertova E, Chertov O, Coren LV, Roser JD, Trubey CM, Bess JW Jr, Sowder RC 2nd, Barsov E, Hood BL, Fisher RJ, Nagashima K, Conrads TP, Veenstra TD, Lifson JD, Ott DE (2006) Proteomic and biochemical analysis of purified human immunodeficiency virus type 1 produced from infected monocyte-derived macrophages. $J$ Virol 80: 9039-9052.

Chien AI, Liao WH, Yang DM, Wang CT (2006) A domain directly C-terminal to the major homology region of human immunodeficiency type 1 capsid protein plays a crucial role in directing both virus assembly and incorporation of Gag-Pol. Virology 348: 84-95.

Chinnasamy D, Milsom MD, Shaffer J, Neuenfeldt J, Shaaban AF, Margison GP, Fairbairn LJ, Chinnasamy N (2006) Multicistronic lentiviral vectors containing the FMDV 2A cleavage factor demonstrate robust expression of encoded genes at limiting MOI. Virol J 3: 14.

Chinnasamy N, Shaffer J, Chinnasamy D (2009) Production of multicistronic HIV-1 based lentiviral vectors. Methods Mol Biol 515: 137-150.

Chiu HC, Wang FD, Chen YM, Wang CT (2006) Effects of human immunodeficiency virus type 1 transframe protein $\mathrm{p}^{*}$ mutations on viral protease-mediated Gag processing. J Gen Virol 87: 2041-2046.

Christ F, Thys W, De Rijck J, Gijsbers R, Albanese A, Arosio D, Emiliani S, Rain JC, Benarous R, Cereseto A, Debyser Z (2008) Transportin-SR2 imports HIV into the nucleus. Curr Biol 18: 1192-1202.

Christensen HS, Daher A, Soye KJ, Frankel LB, Alexander MR, Laine S, Bannwarth S, Ong CL, Chung SW, Campbell SM, Purcell DF, Gatignol A (2007) Small interfering RNAs against the TAR RNA binding protein, TRBP, a Dicer cofactor, inhibit human immunodeficiency virus type 1 long terminal repeat expression and viral production. J Virol 81: 5121-5131.

Chung JH, Whiteley M, Felsenfeld G (1993) A 5' element of the chicken beta-globin domain serves as an insulator in human erythroid cells and protects against position effect in Drosophila. Cell 74: 505-514.

Cicala C, Arthos J, Martinelli E, Censoplano N, Cruz CC, Chung E, Selig SM, Van Ryk D, Yang J, Jagannatha S, Chun TW, Ren P, Lempicki RA, Fauci AS (2006) R5 and X4 HIV envelopes induce distinct gene expression profiles in primary peripheral blood mononuclear cells. Proc Natl Acad Sci USA 103: 3746-3751.

Ciuffi A (2008) Mechanisms governing lentivirus integration site selection. Curr Gene Ther 8: 419-429.

Ciuffi A, Llano M, Poeschla E, Hoffmann C, Leipzig J, Shinn P, Ecker JR, Bushman F (2005) A role for LEDGF/ p75 in targeting HIV DNA integration. Nat Med 11: $1287-1289$.

Clackson T (2000) Regulated gene expression systems. Gene Ther 7: 120-125.

Clever J, Sassetti C, Parslow TG (1995) RNA secondary structure and binding sites for gag gene products in the $5^{\prime}$ packaging signal of human immunodeficiency virus type 1. J Virol 69: 2101-2109.

Cockrell AS, Kafri T (2007) Gene delivery by lentivirus vectors. Mol Biotechnol 36: 184-204.

Cockrell AS, Ma H, Fu K, McCown TJ, Kafri T (2006) A trans-lentiviral packaging cell line for high-titer conditional self-inactivating HIV-1 vectors. Mol Ther 14: 276-284.

Cocohoba J, Dong BJ (2008) Raltegravir: the first HIV integrase inhibitor. Clin Ther 30: 1747-1765.

Coil DA, Miller AD (2004) Phosphatidylserine is not the cell surface receptor for vesicular stomatitis virus. $J \mathrm{Vi}$ rol 78: 10920-10926.

Connolly JB (2002) Lentiviruses in gene therapy clinical research. Gene Ther 9: 1730-1734.

Corbel SY, Rossi FM (2002) Latest developments and in vivo use of the Tet system: ex vivo and in vivo delivery of tetracycline-regulated genes. Curr Opin Biotechnol 13: 448-452.

Cornu TI, Cathomen T (2007) Targeted genome modifications using integrase-deficient lentiviral vectors. $\mathrm{Mol}$ Ther 15: 2107-2113.

Coutant F, Frenkiel MP, Despres P, Charneau P (2008) Protective antiviral immunity conferred by a nonintegrative lentiviral vector-based vaccine. PLoS One 3: e3973.

Cronin J, Zhang XY, Reiser J (2005) Altering the tropism of lentiviral vectors through pseudotyping. Curr Gene Ther 5: 387-398.

Croyle MA, Callahan SM, Auricchio A, Schumer G, Linse KD, Wilson JM, Brunner LJ, Kobinger GP (2004) PEGylation of a vesicular stomatitis virus $G$ pseudotyped lentivirus vector prevents inactivation in serum. J Virol 78: 912-921.

Cui Y, Golob J, Kelleher E, Ye Z, Pardoll D, Cheng L (2002) Targeting transgene expression to antigen-presenting cells derived from lentivirus-transduced engrafting human hematopoietic stem/progenitor cells. Blood 99: 399-408.

Cui Y, Iwakuma T, Chang LJ (1999) Contributions of viral splice sites and cis-regulatory elements to lentivirus vector function. J Virol 73: 6171-6176.

Cui Y, Kelleher E, Straley E, Fuchs E, Gorski K, Levitsky $\mathrm{H}$, Borrello I, Civin CI, Schoenberger SP, Cheng L, Pardoll DM, Whartenby KA (2003) Immunotherapy of established tumors using bone marrow transplantation with antigen gene-modified hematopoietic stem cells. Nat Med 9: 952-958.

Cullen BR (1991) Regulation of HIV-1 gene expression. FASEB I 5: 2361-2368.

Cullen BR (2003) Nuclear mRNA export: insights from virology. Trends Biochem Sci 28: 419-424.

Cullen BR (2009) Viral RNAs: lessons from the enemy. Cell 136: 592-597.

Dagher I, Nguyen TH, Groyer-Picard MT, Lainas P, Mainot S, Guettier C, Pariente D, Franco D, Weber A (2009) Efficient hepatocyte engraftment and long-term transgene expression after reversible portal embolization in nonhuman primates. Hepatology 49: 950-959.

Dalgleish AG, Beverley PC, Clapham PR, Crawford DH, Greaves MF, Weiss RA (1984) The CD4 (T4) antigen is an essential component of the receptor for the AIDS retrovirus. Nature 312: 763-767. 
Daniel R, Smith JA (2008) Integration site selection by retroviral vectors: molecular mechanism and clinical consequences. Hum Gene Ther 19: 557-568.

Dardalhon V, Herpers B, Noraz N, Pflumio F, Guetard D, Leveau C, Dubart-Kupperschmitt A, Charneau P, Taylor N (2001) Lentivirus-mediated gene transfer in primary $\mathrm{T}$ cells is enhanced by a central DNA flap. Gene Ther 8: 190-198.

Das G, Hinkley CS, Herr W (1995) Basal promoter elements as a selective determinant of transcriptional activator function. Nature 374: 657-660.

de Felipe P, Ryan MD (2004) Targeting of proteins derived from self-processing polyproteins containing multiple signal sequences. Traffic 5: 616-626.

Denard J, Rundwasser S, Laroudie N, Gonnet F, Naldini L, Radrizzani M, Galy A, Merten OW, Danos O, Svinartchouk F (2009) Quantitative proteomic analysis of lentiviral vectors using 2-DE. Proteomics 9: 3666-3676.

De Palma M, Mazzieri R, Politi LS, Pucci F, Zonari E, Sitia G, Mazzoleni S, Moi D, Venneri MA, Indraccolo S, Falini A, Guidotti LG, Galli R, Naldini L (2008) Tumortargeted interferon-alpha delivery by Tie2-expressing monocytes inhibits tumor growth and metastasis. Cancer Cell 14: 299-311.

De Palma M, Montini E, Santoni de Sio FR, Benedicenti F, Gentile A, Medico E, Naldini L (2005) Promoter trapping reveals significant differences in integration site selection between MLV and HIV vectors in primary hematopoietic cells. Blood 105: 2307-2315.

De Palma M, Venneri MA, Roca C, Naldini L (2003) Targeting exogenous genes to tumor angiogenesis by transplantation of genetically modified hematopoietic stem cells. Nat Med 9: 789-795.

De Rijck J, Debyser Z (2006) The central DNA flap of the human immunodeficiency virus type 1 is important for viral replication. Biochem Biophys Res Commun 349: 1100-1110.

De Rijck J, Van Maele B, Debyser Z (2005) Positional effects of the central DNA flap in HIV-1-derived lentiviral vectors. Biochem Biophys Res Commun 328: 987-994.

Deichmann A, Hacein-Bey-Abina S, Schmidt M, Garrigue A, Brugman MH, Hu J, Glimm H, Gyapay G, Prum B, Fraser CC, Fischer N, Schwarzwaelder K, Siegler ML, de Ridder D, Pike-Overzet K, Howe SJ, Thrasher AJ, Wagemaker G, Abel U, Staal FJ, Delabesse E, Villeval JL, Aronow B, Hue C, Prinz C, Wissler M, Klanke C, Weissenbach J, Alexander I, Fischer A, von Kalle C, Cavazzana-Calvo M (2007) Vector integration is nonrandom and clustered and influences the fate of lymphopoiesis in SCID-X1 gene therapy. J Clin Invest 117: 2225-2232.

Del Vecchio C, Calistri A, Lombardi G, Celegato M, Biasolo MA, Palu G, Parolin C (2009) Analysis of human immunodeficiency virus type 1 vector cis- and transacting elements production by means of Semliki Forest virus. Gene Ther 16: 279-290.

Delelis O, Carayon K, Saib A, Deprez E, Mouscadet JF (2008) Integrase and integration: biochemical activities of HIV-1 integrase. Retrovirology 5: 114.

Demirov DG, Orenstein JM, Freed EO (2002) The late domain of human immunodeficiency virus type 1 p 6 promotes virus release in a cell type-dependent manner. $J$ Virol 76: 105-117.

DePolo NJ, Reed JD, Sheridan PL, Townsend K, Sauter SL, Jolly DJ, Dubensky TW Jr (2000) VSV-G pseudotyped lentiviral vector particles produced in human cells are inactivated by human serum. Mol Ther 2: 218-222.
Deuschle U, Meyer WK, Thiesen HJ (1995) Tetracycline-reversible silencing of eukaryotic promoters. Mol Cell Biol 15: 1907-1914.

DeWire SM, Kim J, Whalen EJ, Ahn S, Chen M, Lefkowitz RJ (2008) Beta-arrestin-mediated signaling regulates protein synthesis. J Biol Chem 283: 10611-10620.

DeZazzo JD, Scott JM, Imperiale MJ (1992) Relative roles of signals upstream of AAUAAA and promoter proximity in regulation of human immunodeficiency virus type 1 mRNA 3' end formation. Mol Cell Biol 12: 5555-5562.

Dhami H, Fritz CE, Gankin B, Pak SH, Yi W, Seya MJ, Raffa RB, Nagar S (2009) The chemokine system and CCR5 antagonists: potential in HIV treatment and other novel therapies. J Clin Pharm Ther 34: 147-160.

Dismuke DJ, Aiken C (2006) Evidence for a functional link between uncoating of the human immunodeficiency virus type 1 core and nuclear import of the viral preintegration complex. J Virol 80: 3712-3720.

Dobrowsky TM, Zhou Y, Sun SX, Siliciano RF, Wirtz D (2008) Monitoring early fusion dynamics of human immunodeficiency virus type 1 at single-molecule resolution. J Virol 82: 7022-7033.

Donaldson ZR, Yang SH, Chan AW, Young LJ (2009) Production of germline transgenic prairie voles (Microtus ochrogaster) using lentiviral vectors. Biol Reprod 81: 1189-1195.

Dorin D, Bonnet MC, Bannwarth S, Gatignol A, Meurs EF, Vaquero C (2003) The TAR RNA-binding protein, TRBP, stimulates the expression of TAR-containing RNAs in vitro and in vivo independently of its ability to inhibit the dsRNA-dependent kinase PKR. J Biol Chem 278: 4440-4448.

D'Orso I, Frankel AD (2009) Tat acetylation modulates assembly of a viral-host RNA-protein transcription complex. Proc Natl Acad Sci USA 106: 3101-3106.

Dowling D, Nasr-Esfahani S, Tan CH, O'Brien K, Howard JL, Jans DA, Purcell DF, Stoltzfus CM, Sonza S (2008) HIV-1 infection induces changes in expression of cellular splicing factors that regulate alternative viral splicing and virus production in macrophages. Retrovirology 5: 18.

D'Souza V, Summers MF (2004) Structural basis for packaging the dimeric genome of Moloney murine leukaemia virus. Nature 431: 586-590.

Duan Y, Catana A, Meng Y, Yamamoto N, He S, Gupta S, Gambhir SS, Zern MA (2007) Differentiation and enrichment of hepatocyte-like cells from human embryonic stem cells in vitro and in vivo. Stem Cells 25: 3058-3068.

Dull T, Zufferey R, Kelly M, Mandel RJ, Nguyen M, Trono D, Naldini L (1998) A third-generation lentivirus vector with a conditional packaging system. J Virol 72: 8463-8471.

Dullaers M, Breckpot K, Van Meirvenne S, Bonehill A, Tuyaerts S, Michiels A, Straetman L, Heirman C, De Greef C, Van Der Bruggen P, Thielemans K (2004) Side-byside comparison of lentivirally transduced and mRNAelectroporated dendritic cells: implications for cancer immunotherapy protocols. Mol Ther 10: 768-779.

Dürst M, Gissmann L, Ikenberg H, zur Hausen H (1983) A papillomavirus DNA from a cervical carcinoma and its prevalence in cancer biopsy samples from different geographic regions. Proc Natl Acad Sci USA 80: 38123815.

Dvorin JD, Bell P, Maul GG, Yamashita M, Emerman M, Malim MH (2002) Reassessment of the roles of integrase and the central DNA flap in human immunodeficiency virus type 1 nuclear import. J Virol 76: 1208712096. 
Eckstein DA, Penn ML, Korin YD, Scripture-Adams DD, Zack JA, Kreisberg JF, Roederer M, Sherman MP, Chin PS, Goldsmith MA (2001) HIV-1 actively replicates in naive $\mathrm{CD} 4(+) \mathrm{T}$ cells residing within human lymphoid tissues. Immunity 15: 671-682.

Engelman A, Cherepanov P (2008) The lentiviral integrase binding protein LEDGF/p75 and HIV-1 replication. PLoS Pathog 4: e1000046.

European Society of Gene Therapy (ESGT) (2006) One of three successfully treated CGD patients in a Swiss-German gene therapy trial died due to his underlying disease: A position statement from the European Society of Gene Therapy (ESGT) J Gene Med 8: 1435.

Evans JT, Garcia JV (2000) Lentivirus vector mobilization and spread by human immunodeficiency virus. Hum Gene Ther 11: 2331-2339.

Evans-Galea MV, Wielgosz MM, Hanawa H, Srivastava DK, Nienhuis AW (2007) Suppression of clonal dominance in cultured human lymphoid cells by addition of the cHS4 insulator to a lentiviral vector. Mol Ther 15: 801-809.

Farnet CM, Bushman FD (1997) HIV-1 cDNA integration: requirement of HMG $\mathrm{I}(\mathrm{Y})$ protein for function of preintegration complexes in vitro. Cell 88: 483-492.

Farson D, Witt R, McGuinness R, Dull T, Kelly M, Song J, Radeke R, Bukovsky A, Consiglio A, Naldini L (2001) A new-generation stable inducible packaging cell line for lentiviral vectors. Hum Gene Ther 12: 981-997.

Fassati A, Goff SP (1999) Characterization of intracellular reverse transcription complexes of Moloney murine leukemia virus. J Virol 73: 8919-8925.

Federici T, Kutner R, Zhang XY, Kuroda H, Tordo N, Boulis NM, Reiser J (2009) Comparative analysis of HIV-1-based lentiviral vectors bearing lyssavirus glycoproteins for neuronal gene transfer. Genet Vaccines Ther 7: 1.

Felice B, Cattoglio C, Cittaro D, Testa A, Miccio A, Ferrari G, Luzi L, Recchia A, Mavilio F (2009) Transcription factor binding sites are genetic determinants of retroviral integration in the human genome. PLOS ONE 4: e4571.

Feng S, Holland EC (1988) HIV-1 tat trans-activation requires the loop sequence within tar. Nature 334: 165167.

Feng Y, Broder CC, Kennedy PE, Berger EA (1996) HIV1 entry cofactor: functional cDNA cloning of a seventransmembrane, G protein-coupled receptor. Science 272: 872-877.

Finzi A, Orthwein A, Mercier J, Cohen EA (2007b) Productive human immunodeficiency virus type 1 assembly takes place at the plasma membrane. J Virol 81: 74767490.

Finzi D, Dieffenbach CW, Basavappa R (2007a) Defining and solving the essential protein-protein interactions in HIV infection. J Struct Biol 158: 148-155.

Fisher AG, Feinberg MB, Josephs SF, Harper ME, Marselle LM, Reyes G, Gonda MA, Aldovini A, Debouk C, Gallo RC et al. (1986) The trans-activator gene of HTLV-III is essential for virus replication. Nature 320: 367-371.

Flessa S, Marschall P (2009) Socio-economic impact of antiviral intervention. Handb Exp Pharmacol 189: 347-374.

Follenzi A, Battaglia M, Lombardo A, Annoni A, Roncarolo MG, Naldini L (2004) Targeting lentiviral vector expression to hepatocytes limits transgene-specific immune response and establishes long-term expression of human antihemophilic factor IX in mice. Blood 103: 3700-3709.
Fornerod M, Ohno M, Yoshida M, Mattaj IW (1997) CRM1 is an export receptor for leucine-rich nuclear export signals. Cell 90: 1051-1060.

Forsman A, Weiss RA (2008) Why is HIV a pathogen? Trends Microbiol 16: 555-560.

Foster JL, Garcia JV (2008) HIV-1 Nef: at the crossroads. Retrovirology 5: 84.

Frecha C, Costa C, Nègre D, Gauthier E, Russell SJ, Cosset FL, Verhoeyen E (2008a) Stable transduction of quiescent $\mathrm{T}$ cells without induction of cycle progression by a novel lentiviral vector pseudotyped with measles virus glycoproteins. Blood 112: 4843-4852.

Frecha C, Szecsi J, Cosset FL, Verhoeyen E (2008b) Strategies for targeting lentiviral vectors. Curr Gene Ther 8: 449-460.

Frecha C, Costa C, Lévy C, Nègre D, Russell SJ, Maisner A, Salles G, Peng KW, Cosset FL, Verhoeyen E (2009) Efficient and stable transduction of resting B lymphocytes and primary chronic lymphocyte leukemia cells using measles virus gp displaying lentiviral vectors. Blood 114: 3173-3180.

Freed EO (2001) HIV-1 replication. Somat Cell Mol Genet 26: $13-33$.

Freed EO, Englund G, Maldarelli F, Martin MA (1997) Phosphorylation of residue 131 of HIV-1 matrix is not required for macrophage infection. Cell 88: 171-173; discussion 173-174.

Froelich S, Ziegler L, Stroup K, Wang P (2009) Targeted gene delivery to CD117-expressing cells in vivo with lentiviral vectors co-displaying stem cell factor and a fusogenic molecule. Biotechnol Bioeng 104: 206-215

Fu W, Sanders-Beer BE, Katz KS, Maglott DR, Pruitt KD, Ptak RG (2009) Human immunodeficiency virus type 1, human protein interaction database at NCBI. Nucleic Acids Res 37: D417-D422.

Funke S, Maisner A, Mühlebach MD, Koehl U, Grez M, Cattaneo R, Cichutek K, Buchholz CJ (2008) Targeted cell entry of lentiviral vectors. Mol Ther 16: 1427-1436.

Gallay P, Hope T, Chin D, Trono D (1997) HIV-1 infection of nondividing cells through the recognition of integrase by the importin/karyopherin pathway. Proc Natl Acad Sci USA 94: 9825-9830.

Gallay P, Stitt V, Mundy C, Oettinger M, Trono D (1996) Role of the karyopherin pathway in human immunodeficiency virus type 1 nuclear import. J Virol 70: 10271032.

Gallay P, Swingler S, Song J, Bushman F, Trono D (1995) HIV nuclear import is governed by the phosphotyrosine-mediated binding of matrix to the core domain of integrase. Cell 83: 569-576.

Ganser-Pornillos BK, Yeager M, Sundquist WI (2008) The structural biology of HIV assembly. Curr Opin Struct Biol 18: 203-217.

Garber ME, Mayall TP, Suess EM, Meisenhelder J, Thompson NE, Jones KA (2000) CDK9 autophosphorylation regulates high-affinity binding of the human immunodeficiency virus type 1 tat-P-TEFb complex to TAR RNA. Mol Cell Biol 20: 6958-6969.

Gavrilescu LC, Van Etten RA (2007) Production of replication-defective retrovirus by transient transfection of 293T cells. J Vis Exp 550.

Ge H, Si Y, Roeder RG (1998) Isolation of cDNAs encoding novel transcription coactivators p52 and p75 reveals an alternate regulatory mechanism of transcriptional activation. EMBO J 17: 6723-6729.

Gelderblom HC, Vatakis DN, Burke SA, Lawrie SD, Bristol GC, Levy DN (2008) Viral complementation allows HIV-1 replication without integration. Retrovirology 5: 60. 
Geraerts M, Michiels M, Baekelandt V, Debyser Z, Gijsbers $\mathrm{R}$ (2005) Upscaling of lentiviral vector production by tangential flow filtration. J Gene Med 7: 1299-1310.

Geraerts M, Willems S, Baekelandt V, Debyser Z, Gijsbers $R$ (2006) Comparison of lentiviral vector titration methods. BMC Biotechnol 6: 34 .

Gheysen D, Jacobs E, de Foresta F, Thiriart C, Francotte M, Thines D, De Wilde M (1989) Assembly and release of HIV-1 precursor Pr55gag virus-like particles from recombinant baculovirus-infected insect cells. Cell 59: 103-112.

Giannini C, Morosan S, Tralhao JG, Guidotti JE, Battaglia S, Mollier K, Hannoun L, Kremsdorf D, Gilgenkrantz H, Charneau P (2003) A highly efficient, stable, and rapid approach for ex vivo human liver gene therapy via a FLAP lentiviral vector. Hepatology 38: 114-122.

Gibbs JS, Regier DA, Desrosiers RC (1994) Construction and in vitro properties of HIV-1 mutants with deletions in "nonessential" genes. AIDS Res Hum Retroviruses 10: 343-350.

Giedroc DP, Cornish PV (2009) Frameshifting RNA pseudoknots: structure and mechanism. Virus Res 139: 193208.

Gillim-Ross L, Cara A, Klotman ME (2005) HIV-1 extrachromosomal 2-LTR circular DNA is long-lived in human macrophages. Viral Immunol 18: 190-196.

Goh WC, Rogel ME, Kinsey CM, Michael SF, Fultz PN, Nowak MA, Hahn BH, Emerman M (1998) HIV-1 Vpr increases viral expression by manipulation of the cell cycle: a mechanism for selection of $\mathrm{Vpr}$ in vivo. Nat Med 4: 65-71.

Goila-Gaur R, Strebel K (2008) HIV-1 Vif, APOBEC, and intrinsic immunity. Retrovirology 5: 51 .

Gomez MC, Pope CE, Kutner RH, Ricks DM, Lyons LA, Ruhe MT, Dumas C, Lyons J, Dresser BL, Reiser J (2009) Generation of domestic transgenic cloned kittens using lentivirus vectors. Cloning Stem Cells 11: 167-176.

Gonda MA, Wong-Staal F, Gallo RC, Clements JE, Narayan O, Gilden RV (1985) Sequence homology and morphologic similarity of HTLV-III and visna virus, a pathogenic lentivirus. Science 227: 173-177.

Gossen M, Bujard H (1992) Tight control of gene expression in mammalian cells by tetracycline-responsive promoters. Proc Natl Acad Sci USA 89: 5547-5551.

Gossen M, Bujard H (2002) Studying gene function in eukaryotes by conditional gene inactivation. Annu Rev Genet 36: 153-173.

Gossen M, Freundlieb S, Bender G, Muller G, Hillen W, Bujard H (1995) Transcriptional activation by tetracyclines in mammalian cells. Science 268: 1766-1769.

Gottlinger HG, Dorfman T, Sodroski JG, Haseltine WA (1991) Effect of mutations affecting the p6 gag protein on human immunodeficiency virus particle release. Proc Natl Acad Sci USA 88: 3195-3199.

Gould SJ, Booth AM, Hildreth JE (2003) The Trojan exosome hypothesis. Proc Natl Acad Sci USA 100: 1059210597.

Goverdhana S, Puntel M, Xiong W, Zirger JM, Barcia C, Curtin JF, Soffer EB, Mondkar S, King GD, Hu J, Sciascia SA, Candolfi M, Greengold DS, Lowenstein PR, Castro MG (2005) Regulatable gene expression systems for gene therapy applications: progress and future challenges. Mol Ther 12: 189-211.

Green DS, Center DM, Cruikshank WW (2009) HIV-1 gp120 reprogramming of CD4+ $\mathrm{T}$ cell migration provides a mechanism for lymphadenopathy. J Virol 83: 5765-5772.

Greenberg KP, Geller SF, Schaffer DV, Flannery JG (2007) Targeted transgene expression in muller glia of nor- mal and diseased retinas using lentiviral vectors. Invest Ophthalmol Vis Sci 48: 1844-1852.

Griffin SD, Allen JF, Lever AM (2001) The major human immunodeficiency virus type 2 (HIV-2) packaging signal is present on all HIV-2 RNA species: cotranslational RNA encapsidation and limitation of Gag protein confer specificity. J Virol 75: 12058-12069.

Groom HC, Anderson EC, Dangerfield JA, Lever AM (2009) Rev regulates translation of human immunodeficiency virus type 1 RNAs. J Gen Virol 90: 1141-1147.

Gruh I, Wunderlich S, Winkler M, Schwanke K, Heinke J, Blomer U, Ruhparwar A, Rohde B, Li RK, Haverich A, Martin U (2008) Human CMV immediate-early enhancer: a useful tool to enhance cell-type-specific expression from lentiviral vectors. J Gene Med 10: 21-32.

Guo J, Wu T, Anderson J, Kane BF, Johnson DG, Gorelick RJ, Henderson LE, Levin JG (2000) Zinc finger structures in the human immunodeficiency virus type $1 \mathrm{nu}-$ cleocapsid protein facilitate efficient minus- and plusstrand transfer. I Virol 74: 8980-8988.

Gupta S, Schoer RA, Egan JE, Hannon GJ, Mittal V (2004) Inducible, reversible, and stable RNA interference in mammalian cells. Proc Natl Acad Sci USA 101: 19271932.

Hacein-Bey-Abina S, Garrigue A, Wang GP, Soulier J, Lim A, Morillon E, Clappier E, Caccavelli L, Delabesse E, Beldjord K, Asnafi V, MacIntyre E, Dal Cortivo L, Radford I, Brousse N, Sigaux F, Moshous D, Hauer J, Borkhardt A, Belohradsky BH, Wintergerst U, Velez MC, Leiva L, Sorensen R, Wulffraat N, Blanche S, Bushman FD, Fischer A, Cavazzana-Calvo M (2008) Insertional oncogenesis in 4 patients after retrovirusmediated gene therapy of SCID-X1. J Clin Invest 118: 3132-3142.

Hacein-Bey-Abina S, von Kalle C, Schmidt M, Le Deist F, Wulffraat N, McIntyre E, Radford I, Villeval JL, Fraser CC, Cavazzana-Calvo M, Fischer A (2003) A serious adverse event after successful gene therapy for X-linked severe combined immunodeficiency. $N$ Engl J Med 348: 255-256.

Hager S, Frame FM, Collins AT, Burns JE, Maitland NJ (2008) An internal polyadenylation signal substantially increases expression levels of lentivirus-delivered transgenes but has the potential to reduce viral titer in a promoter-dependent manner. Hum Gene Ther 19: 840-850.

Han Y, Wang X, Dang Y, Zheng YH (2008) APOBEC3G and APOBEC3F require an endogenous cofactor to block HIV-1 replication. PLoS Pathog 4: e1000095.

Hanawa H, Persons DA, Nienhuis AW (2002) High-level erythroid lineage-directed gene expression using globin gene regulatory elements after lentiviral vector-mediated gene transfer into primitive human and murine hematopoietic cells. Hum Gene Ther 13: 2007-2016.

Hanawa H, Persons DA, Nienhuis AW (2005) Mobilization and mechanism of transcription of integrated self-inactivating lentiviral vectors. J Virol 79: 8410-8421.

Hanawa $\mathrm{H}$, Yamamoto $\mathrm{M}$, Zhao $\mathrm{H}$, Shimada $\mathrm{T}$, Persons DA (2009) Optimized lentiviral vector design improves titer and transgene expression of vectors containing the chicken beta-globin locus HS4 insulator element. Mol Ther 17: 667-674.

Harris RS, Bishop KN, Sheehy AM, Craig HM, PetersenMahrt SK, Watt IN, Neuberger MS, Malim MH (2003) DNA deamination mediates innate immunity to retroviral infection. Cell 113: 803-809.

He Z, Zhang W, Chen G, Xu R, Yu XF (2008) Characterization of conserved motifs in HIV-1 Vif required for 
APOBEC3G and APOBEC3F interaction. I Mol Biol 381: 1000-1011.

Heldt SA, Stanek L, Chhatwal JP, Ressler KJ (2007) Hippocampus-specific deletion of BDNF in adult mice impairs spatial memory and extinction of aversive memories. Mol Psychiatry 12: 656-670.

Helseth E, Kowalski M, Gabuzda D, Olshevsky U, Haseltine W, Sodroski J (1990) Rapid complementation assays measuring replicative potential of human immunodeficiency virus type 1 envelope glycoprotein mutants. J Virol 64: 2416-2420.

Herbeuval JP, Grivel JC, Boasso A, Hardy AW, Chougnet C, Dolan MJ, Yagita H, Lifson JD, Shearer GM (2005) CD4+ T-cell death induced by infectious and noninfectious HIV-1: role of type 1 interferon-dependent, TRAIL/DR5-mediated apoptosis. Blood 106: 3524-3531.

Herbeuval JP, Shearer GM (2007) HIV-1 immunopathogenesis: how good interferon turns bad. Clin Immunol 123: 121-128.

Herbreteau CH, Weill L, Decimo D, Prevot D, Darlix JL, Sargueil B, Ohlmann T (2005) HIV-2 genomic RNA contains a novel type of IRES located downstream of its initiation codon. Nat Struct Mol Biol 12: 1001-1007.

Higashikawa F, Chang L (2001) Kinetic analyses of stability of simple and complex retroviral vectors. Virology 280: 124-131.

Higashimoto T, Urbinati F, Perumbeti A, Jiang G, Zarzuela A, Chang LJ, Kohn DB, Malik P (2007) The woodchuck hepatitis virus post-transcriptional regulatory element reduces readthrough transcription from retroviral vectors. Gene Ther 14: 1298-1304.

Hindmarsh P, Ridky T, Reeves R, Andrake M, Skalka AM, Leis J (1999) HMG protein family members stimulate human immunodeficiency virus type 1 and avian sarcoma virus concerted DNA integration in vitro. J Virol 73: 2994-3003.

Hino S, Fan J, Taguwa S, Akasaka K, Matsuoka M (2004) Sea urchin insulator protects lentiviral vector from silencing by maintaining active chromatin structure. Gene Ther 11: 819-828.

Hioki H, Kuramoto E, Konno M, Kameda H, Takahashi Y, Nakano T, Nakamura KC, Kaneko T (2009) High-level transgene expression in neurons by lentivirus with TetOff system. Neurosci Res 63: 149-154.

Hitz C, Steuber-Buchberger P, Delic S, Wurst W, Kuhn R (2009) Generation of shRNA transgenic mice. Methods Mol Biol 530: 101-129.

Hitz C, Wurst W, Kuhn R (2007) Conditional brain-specific knockdown of MAPK using Cre/loxP regulated RNA interference. Nucleic Acids Res 35: e90.

Hockemeyer D, Soldner F, Cook EG, Gao Q, Mitalipova M, Jaenisch R (2008) A drug-inducible system for direct reprogramming of human somatic cells to pluripotency. Cell Stem Cell 3: 346-353.

Ho DD, Bieniasz PD (2008) HIV-1 at 25. Cell 133: 561-565.

Hofmann A, Wenzel D, Becher UM, Freitag DF, Klein AM, Eberbeck D, Schulte M, Zimmermann K, Bergemann C, Gleich B, Roell W, Weyh T, Trahms L, Nickenig G, Fleischmann BK, Pfeifer A (2009) Combined targeting of lentiviral vectors and positioning of transduced cells by magnetic nanoparticles. Proc Natl Acad Sci USA 106: $44-49$.

Holman AG, Coffin JM (2005) Symmetrical base preferences surrounding HIV-1, avian sarcoma/leukosis virus, and murine leukemia virus integration sites. Proc Natl Acad Sci USA 102: 6103-6107.

Hombrouck A, De Rijck J, Hendrix J, Vandekerckhove L, Voet A, De Maeyer M, Witvrouw M, Engelborghs Y, Christ F, Gijsbers R, Debyser Z (2007) Virus evolution reveals an exclusive role for LEDGF/p75 in chromosomal tethering of HIV. PLoS Pathog 3: e47.

Hu B, Yang H, Dai B, Tai A, Wang P (2009b) Nonintegrating lentiviral vectors can effectively deliver ovalbumin antigen for induction of antitumor immunity. Hum Gene Ther 00: 000-000.?

Hu T, Fu Q, Chen P, Zhang K, Guo D (2009a) Generation of a stable mammalian cell line for simultaneous expression of multiple genes by using $2 \mathrm{~A}$ peptide-based lentiviral vector. Biotechnol Lett 31: 353-359.

Huang M, Orenstein JM, Martin MA, Freed EO (1995) p6Gag is required for particle production from fulllength human immunodeficiency virus type 1 molecular clones expressing protease. J Virol 69: 6810-6818.

Huthoff H, Towers GJ (2008) Restriction of retroviral replication by APOBEC3G/F and TRIM5alpha. Trends Microbiol 16: 612-619.

Ibrahimi A, Velde GV, Reumers V, Toelen J, Thiry I, Vandeputte C, Vets S, Deroose C, Bormans G, Baekelandt V, Debyser Z, Gijsbers R (2009) Highly efficient multicistronic lentiviral vectors with peptide $2 \mathrm{~A}$ sequences. Hum Gene Ther 20: 845-860.

Iwakuma T, Cui Y, Chang LJ (1999) Self-inactivating lentiviral vectors with U3 and U5 modifications. Virology 261: 120-132.

Iyer SR, Yu D, Biancotto A, Margolis LB, Wu Y (2009) Measurement of human immunodeficiency virus type 1 preintegration transcription by using Rev-dependent Rev-CEM cells reveals a sizable transcribing DNA population comparable to that from proviral templates. J Virol 83: 8662-8673.

Jablonski JA, Caputi M (2009) Role of cellular RNA processing factors in human immunodeficiency virus type $1 \mathrm{mRNA}$ metabolism, replication, and infectivity. J Virol 83: 981-992.

Jacks T, Power MD, Masiarz FR, Luciw PA, Barr PJ, Varmus HE (1988) Characterization of ribosomal frameshifting in HIV-1 gag-pol expression. Nature 331: 280-283.

Johansen J, Rosenblad C, Andsberg K, Moller A, Lundberg C, Bjorlund A, Johansen TE (2002) Evaluation of Tet-on system to avoid transgene down-regulation in ex vivo gene transfer to the CNS. Gene Ther 9: 1291-1301.

Jolly C, Kashefi K, Hollinshead M, Sattentau QJ (2004) HIV-1 cell to cell transfer across an Env-induced, actindependent synapse. J Exp Med 199: 283-293.

Jolly C, Mitar I, Sattentau QJ (2007) Requirement for an intact T-cell actin and tubulin cytoskeleton for efficient assembly and spread of human immunodeficiency virus type 1. J Virol 81: 5547-5560.

Jolly C, Sattentau QJ (2005) Human immunodeficiency virus type 1 virological synapse formation in T cells requires lipid raft integrity. J Virol 79: 12088-12094.

Joshi A, Ablan SD, Soheilian F, Nagashima K, Freed EO (2009) Evidence that productive human immunodeficiency virus type 1 assembly can occur in an intracellular compartment. J Virol 83: 5375-5387.

Kafaie J, Dolatshahi M, Ajamian L, Song R, Mouland AJ, Rouiller I, Laughrea M (2009) Role of capsid sequence and immature nucleocapsid proteins $\mathrm{p} 9$ and $\mathrm{p} 15$ in human immunodeficiency virus type 1 genomic RNA dimerization. Virology 385: 233-244.

Kafaie J, Song R, Abrahamyan L, Mouland AJ, Laughrea M (2008) Mapping of nucleocapsid residues important for HIV-1 genomic RNA dimerization and packaging. Virology 375: 592-610.

Kafri T, Blomer U, Peterson DA, Gage FH, Verma IM (1997) Sustained expression of genes delivered directly 
into liver and muscle by lentiviral vectors. Nat Genet 17: 314-317.

Kafri T, van Praag H, Gage FH, Verma IM (2000) Lentiviral vectors: regulated gene expression. Mol Ther 1: 516-521.

Kafri T, van Praag H, Ouyang L, Gage FH, Verma IM (1999) A packaging cell line for lentivirus vectors. J Virol 73: 576-584.

Kalpana GV, Marmon S, Wang W, Crabtree GR, Goff SP (1994) Binding and stimulation of HIV-1 integrase by a human homolog of yeast transcription factor SNF5. Science 266: 2002-2006.

Kang SM, Compans RW (2009) Host responses from innate to adaptive immunity after vaccination: molecular and cellular events. Mol Cells 27: 5-14.

Kao S, Goila-Gaur R, Miyagi E, Khan MA, Opi S, Takeuchi H, Strebel K (2007) Production of infectious virus and degradation of APOBEC $3 \mathrm{G}$ are separable functional properties of human immunodeficiency virus type 1 Vif. Virology 369: 329-339.

Kao SY, Calman AF, Luciw PA, Peterlin BM (1987) Antitermination of transcription within the long terminal repeat of HIV-1 by tat gene product. Nature 330: 489493.

Kappes JC, Wu X, Wakefield JK (2003) Production of translentiviral vector with predictable safety. Methods $\mathrm{Mol}$ Med 76: 449-465.

Karolewski BA, Watson DJ, Parente MK, Wolfe JH (2003) Comparison of transfection conditions for a lentivirus vector produced in large volumes. Hum Gene Ther 14: 1287-1296.

Karwacz K, Mukherjee S, Apolonia L, Blundell MP, Bouma G, Escors D, Collins MK, Thrasher AJ (2009) Nonintegrating lentivector vaccines stimulate prolonged $\mathrm{T}$ cell and antibody responses and are effective in tumor therapy. J Virol 83: 3094-3103.

Kaye JF, Lever AM (1999) Human immunodeficiency virus types 1 and 2 differ in the predominant mechanism used for selection of genomic RNA for encapsidation. J Virol 73: 3023-3031.

Khare PD, Loewen N, Teo W, Barraza RA, Saenz DT, Johnson DH, Poeschla EM (2008) Durable, safe, multi-gene lentiviral vector expression in feline trabecular meshwork. Mol Ther 16: 97-106.

Kiernan RE, Vanhulle C, Schiltz L, Adam E, Xiao H, Maudoux F, Calomme C, Burny A, Nakatani Y, Jeang KT, Benkirane M, Van Lint C (1999) HIV-1 tat transcriptional activity is regulated by acetylation. EMBO J 18: 6106-6118.

Kim VN, Mitrophanous K, Kingsman SM, Kingsman AJ (1998) Minimal requirement for a lentivirus vector based on human immunodeficiency virus type $1 . \mathrm{J} \mathrm{Vi}$ rol 72: 811-816.

Kim Y, Tewari M, Pajerowski JD, Cai S, Sen S, Williams J, Sirsi S, Lutz G, Discher DE (2009) Polymersome delivery of siRNA and antisense oligonucleotides. J Control Release 134: 132-140.

Klages N, Zufferey R, Trono D (2000) A stable system for the high-titer production of multiply attenuated lentiviral vectors. Mol Ther 2: 170-176.

Klimas N, Koneru AO, Fletcher MA (2008) Overview of HIV. Psychosom Med 70: 523-530.

Klinghoffer RA, Roberts B, Annis J, Frazier J, Lewis P, Linsley PS, Cleary MA (2008) An optimized lentivirusmediated RNAi screen reveals kinase modulators of kinesin-5 inhibitor sensitivity. Assay Drug Dev Technol 6: 105-119.

Klinghoffer RA, Frazier J, Annis J, Berndt JD, Roberts BS, Arthur WT, Lacson R, Zhang XD, Ferrer M, Moon RT,
Cleary MA (2009) A lentivirus-mediated genetic screen identifies dihydrofolate reductase (DHFR) as a modulator of beta-catenin/GSK3 signaling. PLoS One 4: e6892.

Kobayashi M, Takaori-Kondo A, Miyauchi Y, Iwai K, Uchiyama T (2005) Ubiquitination of APOBEC3G by an HIV-1 Vif-Cullin5-Elongin B-Elongin C complex is essential for Vif function. J Biol Chem 280: 18573-18578.

Kolegraff K, Bostik P, Ansari AA (2006) Characterization and role of lentivirus-associated host proteins. Exp Biol Med (Maywood) 231: 252-263.

Komano J, Miyauchi K, Matsuda Z, Yamamoto N (2004) Inhibiting the Arp2/3 complex limits infection of both intracellular mature vaccinia virus and primate lentiviruses. Mol Biol Cell 15: 5197-5207.

Koponen JK, Kankkonen H, Kannasto J, Wirth T, Hillen W, Bujard H, Yla-Herttuala S (2003) Doxycycline-regulated lentiviral vector system with a novel reverse transactivator rtTA2S-M2 shows a tight control of gene expression in vitro and in vivo. Gene Ther 10: 459-466.

Kotsopoulou E, Kim VN, Kingsman AJ, Kingsman SM, Mitrophanous KA (2000) A Rev-independent human immunodeficiency virus type 1 (HIV-1)-based vector that exploits a codon-optimized HIV-1 gag-pol gene. $J$ Virol 74: 4839-4852.

Kramer B, Pelchen-Matthews A, Deneka M, Garcia E, Piguet V, Marsh M (2005) HIV interaction with endosomes in macrophages and dendritic cells. Blood Cells Mol Dis 35: 136-142.

Kringstein AM, Rossi FM, Hofmann A, Blau HM (1998) Graded transcriptional response to different concentrations of a single transactivator. Proc Natl Acad Sci USA 95: 13670-13675.

Kuate S, Stefanou D, Hoffmann D, Wildner O, Uberla K (2004) Production of lentiviral vectors by transient expression of minimal packaging genes from recombinant adenoviruses. J Gene Med 6: 1197-1205.

Kumar M, Keller B, Makalou N, Sutton RE (2001) Systematic determination of the packaging limit of lentiviral vectors. Hum Gene Ther 12: 1893-1905.

Kuroda H, Kutner RH, Bazan NG, Reiser J (2008) A comparative analysis of constitutive and cell-specific promoters in the adult mouse hippocampus using lentivirus vector-mediated gene transfer. J Gene Med 10: 1163-1175.

Kuroda H, Kutner RH, Bazan NG, Reiser J (2009) Simplified lentivirus vector production in protein-free media using polyethylenimine-mediated transfection. J Virol Methods 157: 113-121.

Kurre P, Anandakumar P, Kiem HP (2006) Rapid 1-hour transduction of whole bone marrow leads to long-term repopulation of murine recipients with lentivirus-modified hematopoietic stem cells. Gene Ther 13: 369-373.

Kutner RH, Puthli S, Marino MP, Reiser J (2009a) Simplified production and concentration of HIV-1-based lentiviral vectors using HYPERFlask vessels and anion exchange membrane chromatography. BMC Biotechnol 9: 10.

Kutner RH, Zhang XY, Reiser J (2009b) Production, concentration and titration of pseudotyped HIV-1-based lentiviral vectors. Nat Protoc 4: 495-505.

Laguette N, Benichou S, Basmaciogullari S (2009) Human immunodeficiency virus type 1 Nef incorporation into virions does not increase infectivity. J Virol 83: 10931104.

Lai Z, Brady RO (2002) Gene transfer into the central nervous system in vivo using a recombinanat lentivirus vector. J Neurosci Res 67: 363-371.

Landau NR, Page KA, Littman DR (1991) Pseudotyping with human T-cell leukemia virus type I broadens the 
human immunodeficiency virus host range. J Virol 65: 162-169.

Landau NR, Warton M, Littman DR (1988) The envelope glycoprotein of the human immunodeficiency virus binds to the immunoglobulin-like domain of CD4. $\mathrm{Na}$ ture 334: 159-162.

Laufs S, Guenechea G, Gonzalez-Murillo A, Zsuzsanna Nagy K, Luz Lozano M, del Val C, Jonnakuty S, HotzWagenblatt A, Jens Zeller W, Bueren JA, Fruehauf $S$ (2006) Lentiviral vector integration sites in human NOD/SCID repopulating cells. J Gene Med 8: 11971207.

Le Rouzic E, Benichou S (2005) The Vpr protein from HIV1: distinct roles along the viral life cycle. Retrovirology 2: 11.

Lee HJ, Lee YS, Kim HS, Kim YK, Kim JH, Jeon SH, Lee HW, Kim S, Miyoshi H, Chung HM, Kim DK (2009) Retronectin enhances lentivirus-mediated gene delivery into hematopoietic progenitor cells. Biologicals 37: 203-209.

Lei Y, Joo KI, Wang P (2009) Engineering fusogenic molecules to achieve targeted transduction of enveloped lentiviral vectors. J Biol Eng 3: 8.

Lemiale F, Korokhov N (2009) Lentiviral vectors for HIV disease prevention and treatment. Vaccine 27: 34433449.

Lesch HP, Turpeinen S, Niskanen EA, Mahonen AJ, Airenne KJ, Yla-Herttuala S (2008) Generation of lentivirus vectors using recombinant baculoviruses. Gene Ther 15: $1280-1286$

Levin A, Hayouka Z, Helfer M, Brack-Werner R, Friedler A, Loyter A (2009) Peptides derived from HIV-1 integrase that bind Rev stimulate viral genome integration. PLOS ONE 4: e4155.

Levine BL, Humeau LM, Boyer J, MacGregor RR, Rebello T, Lu X, Binder GK, Slepushkin V, Lemiale F, Mascola JR, Bushman FD, Dropulic B, June CH (2006) Gene transfer in humans using a conditionally replicating lentiviral vector. Proc Natl Acad Sci USA 103: 1737217377.

Lewinski MK, Yamashita M, Emerman M, Ciuffi A, Marshall H, Crawford G, Collins F, Shinn P, Leipzig J, Hannenhalli S, Berry CC, Ecker JR, Bushman FD (2006) Retroviral DNA integration: viral and cellular determinants of target-site selection. PLoS Pathog 2: e60.

Lewis PF, Emerman M (1994) Passage through mitosis is required for oncoretroviruses but not for the human immunodeficiency virus. J Virol 68: 510-516.

Li L, Olvera JM, Yoder KE, Mitchell RS, Butler SL, Lieber M, Martin SL, Bushman FD (2001) Role of the non-homologous DNA end joining pathway in the early steps of retroviral infection. EMBO J 20: 3272-3281.

Li M, Craigie R (2009) Nucleoprotein complex intermediates in HIV-1 integration. Methods 47: 237-242.

Li Y, Drone C, Sat E, Ghosh HP (1993) Mutational analysis of the vesicular stomatitis virus glycoprotein $G$ for membrane fusion domains. J Virol 67: 4070-4077.

Lieberman-Blum SS, Fung HB, Bandres JC (2008) Maraviroc: a CCR5-receptor antagonist for the treatment of HIV-1 infection. Clin Ther 30: 1228-1250.

Lillico SG, Sherman A, McGrew MJ, Robertson CD, Smith J, Haslam C, Barnard P, Radcliffe PA, Mitrophanous KA, Elliot EA, Sang HM (2007) Oviduct-specific expression of two therapeutic proteins in transgenic hens. Proc Natl Acad Sci USA 104: 1771-1776.

Limberis MP, Bell CL, Heath J, Wilson JM (2009) Activation of Transgene-specific T Cells Following Lentivirusmediated Gene Delivery to Mouse Lung. Mol Ther 00: 000-000.?
Limon A, Devroe E, Lu R, Ghory HZ, Silver PA, Engelman A (2002a) Nuclear localization of human immunodeficiency virus type 1 preintegration complexes (PICs): V165A and R166A are pleiotropic integrase mutants primarily defective for integration, not PIC nuclear import. J Virol 76: 10598-10607.

Limon A, Nakajima N, Lu R, Ghory HZ, Engelman A (2002b) Wild-type levels of nuclear localization and human immunodeficiency virus type 1 replication in the absence of the central DNA flap. J Virol 76: 1207812086.

Lin CW, Engelman A (2003) The barrier-to-autointegration factor is a component of functional human immunodeficiency virus type 1 preintegration complexes. J Virol 77: 5030-5036.

Lin X, Yang J, Chen J, Gunasekera A, Fesik SW, Shen Y (2004) Development of a tightly regulated U6 promoter for shRNA expression. FEBS Lett 577: 376-380.

Liovat AS, Jacquelin B, Ploquin MJ, Barre-Sinoussi F, Muller-Trutwin MC (2009) African non human primates infected by SIV - why don't they get sick? Lessons from studies on the early phase of non-pathogenic SIV infection. Curr HIV Res 7: 39-50.

Liu B, Paton JF, Kasparov S (2008) Viral vectors based on bidirectional cell-specific mammalian promoters and transcriptional amplification strategy for use in vitro and in vivo. BMC Biotechnol 8: 49.

Liu B, Sarkis PT, Luo K, Yu Y, Yu XF (2005) Regulation of Apobec3F and human immunodeficiency virus type 1 Vif by Vif-Cul5-ElonB/C E3 ubiquitin ligase. J Virol 79: 9579-9587.

Liu R, Paxton WA, Choe S, Ceradini D, Martin SR, Horuk R, MacDonald ME, Stuhlmann H, Koup RA, Landau NR (1996) Homozygous defect in HIV-1 coreceptor accounts for resistance of some multiply-exposed individuals to HIV-1 infection. Cell 86: 367-377.

Lizee G, Aerts JL, Gonzales MI, Chinnasamy N, Morgan RA, Topalian SL (2003) Real-time quantitative reverse transcriptase-polymerase chain reaction as a method for determining lentiviral vector titers, measuring transgene expression. Hum Gene Ther 14: 497-507.

Llano M, Gaznick N, Poeschla EM (2009) Rapid, controlled and intensive lentiviral vector-based RNAi. Methods Mol Biol 485: 257-270.

Llano M, Vanegas M, Fregoso O, Saenz D, Chung S, Peretz M, Poeschla EM (2004) LEDGF/p75 determines cellular trafficking of diverse lentiviral but not murine oncoretroviral integrase proteins and is a component of functional lentiviral preintegration complexes. J Virol 78: 9524-9537.

Llano M, Vanegas M, Hutchins N, Thompson D, Delgado S, Poeschla EM (2006) Identification and characterization of the chromatin-binding domains of the HIV-1 integrase interactor LEDGF/p75. J Mol Biol 360: 760-773.

Locher CP, Witt SA, Kassel R, Dowell NL, Fujimura S, Levy JA (2005) Differential effects of R5 and X4 human immunodeficiency virus type 1 infection on CD4+ cell proliferation and activation. J Gen Virol 86: 1171-1179.

Logan AC, Haas DL, Kafri T, Kohn DB (2004a) Integrated self-inactivating lentiviral vectors produce full-length genomic transcripts competent for encapsidation and integration. J Virol 78: 8421-8436.

Logan AC, Nightingale SJ, Haas DL, Cho GJ, Pepper KA, Kohn DB (2004b) Factors influencing the titer and infectivity of lentiviral vectors. Hum Gene Ther 15: 976988.

Lois C, Hong EJ, Pease S, Brown EJ, Baltimore D (2002) Germline transmission and tissue-specific expression of 
transgenes delivered by lentiviral vectors. Science 295: 868-872.

Lombardo A, Genovese P, Beausejour CM, Colleoni S, Lee YL, Kim KA, Ando D, Urnov FD, Galli C, Gregory PD, Holmes MC, Naldini L (2007) Gene editing in human stem cells using zinc finger nucleases and integrasedefective lentiviral vector delivery. Nat Biotechnol 25: 1298-1306.

Louis JM, Ishima R, Torchia DA, Weber IT (2007) HIV-1 protease: structure, dynamics, and inhibition. Adv Pharmacol 55: 261-298.

Lourenco S, Boni S, Furling D, Cosset FL, Cahour A (2009) A cell-based bicistronic lentiviral reporter system for identification of inhibitors of the hepatitis $C$ virus internal ribosome entry site. J Virol Methods 158: 152-159.

Lu X, Humeau L, Slepushkin V, Binder G, Yu Q, Slepushkina T, Chen Z, Merling R, Davis B, Chang YN, Dropulic B (2004) Safe two-plasmid production for the first clinical lentivirus vector that achieves $>99 \%$ transduction in primary cells using a one-step protocol. J Gene Med 6: 963-973.

Ma H, Kafri T (2004) A single-LTR HIV-1 vector optimized for functional genomics applications. Mol Ther 10: 139149.

MacGregor RR (2001) Clinical protocol. A phase 1 openlabel clinical trial of the safety and tolerability of single escalating doses of autologous CD4 T cells transduced with VRX496 in HIV-positive subjects. Hum Gene Ther 12: 2028-2029.

Maertens G, Cherepanov P, Pluymers W, Busschots K, De Clercq E, Debyser Z, Engelborghs Y (2003) LEDGF/p75 is essential for nuclear and chromosomal targeting of HIV-1 integrase in human cells. J Biol Chem 278: 3352833539.

Maherali N, Ahfeldt T, Rigamonti A, Utikal J, Cowan C, Hochedlinger K (2008) A high-efficiency system for the generation and study of human induced pluripotent stem cells. Cell Stem Cell 3: 340-345.

Majumder B, Venkatachari NJ, Srinivasan A, Ayyavoo V (2009) HIV-1 mediated immune pathogenesis: spotlight on the role of viral protein $\mathrm{R}(\mathrm{Vpr})$ Curr HIV Res 7: 169-177.

Malim MH (2009) APOBEC proteins and intrinsic resistance to HIV-1 infection. Philos Trans $R$ Soc Lond B Biol Sci 364: 675-687.

Malim MH, Hauber J, Le SY, Maizel JV, Cullen BR (1989) The HIV-1 rev trans-activator acts through a structured target sequence to activate nuclear export of unspliced viral mRNA. Nature 338: 254-257.

Manganini M, Serafini M, Bambacioni F, Casati C, Erba E, Follenzi A, Naldini L, Bernasconi S, Gaipa G, Rambaldi A, Biondi A, Golay J, Introna M (2002) A human immunodeficiency virus type 1 pol gene-derived sequence (cPPT/CTS) increases the efficiency of transduction of human nondividing monocytes and T lymphocytes by lentiviral vectors. Hum Gene Ther 13: 1793-1807.

Manilla P, Rebello T, Afable C, Lu X, Slepushkin V, Humeau LM, Schonely K, Ni Y, Binder GK, Levine BL, MacGregor RR, June CH, Dropulic B (2005) Regulatory considerations for novel gene therapy products: a review of the process leading to the first clinical lentiviral vector. Hum Gene Ther 16: 17-25.

Manjunath N, Wu H, Subramanya S, Shankar P (2009) Lentiviral delivery of short hairpin RNAs. Adv Drug Deliv Rev 61: 732-745.

Mann R, Mulligan RC, Baltimore D (1983) Construction of a retrovirus packaging mutant and its use to produce helper-free defective retrovirus. Cell 33: 153-159.
Mansharamani M, Graham DR, Monie D, Lee KK, Hildreth JE, Siliciano RF, Wilson KL (2003) Barrier-to-autointegration factor BAF binds p55 Gag and matrix and is a host component of human immunodeficiency virus type 1 virions. J Virol 77: 13084-13092.

Margolin JF, Friedman JR, Meyer WK, Vissing H, Thiesen HJ, Rauscher FJ 3rd (1994) Kruppel-associated boxes are potent transcriptional repression domains. Proc Natl Acad Sci USA 91: 4509-4513.

Mariani R, Chen D, Schrofelbauer B, Navarro F, Konig R, Bollman B, Munk C, Nymark-McMahon H, Landau NR (2003) Species-specific exclusion of APOBEC3G from HIV-1 virions by Vif. Cell 114: 21-31.

Marino MP, Luce MJ, Reiser J (2003) Small- to large-scale production of lentivirus vectors. Methods Mol Biol 229: 43-55.

Markosyan RM, Cohen FS, Melikyan GB (2003) HIV-1 envelope proteins complete their folding into six-helix bundles immediately after fusion pore formation. Mol Biol Cell 14: 926-938.

Marshall HM, Ronen K, Berry C, Llano M, Sutherland H, Saenz D, Bickmore W, Poeschla E, Bushman FD (2007) Role of PSIP1/LEDGF/p75 in lentiviral infectivity and integration targeting. PLoS ONE 2: e1340.

Mazarakis ND, Azzouz M, Rohll JB, Ellard FM, Wilkes FJ, Olsen AL, Carter EE, Barber RD, Baban DF, Kingsman SM, Kingsman AJ, O'Malley K, Mitrophanous KA (2001) Rabies virus glycoprotein pseudotyping of lentiviral vectors enables retrograde axonal transport and access to the nervous system after peripheral delivery. Hum Mol Genet 10: 2109-2121.

McDonald D, Vodicka MA, Lucero G, Svitkina TM, Borisy GG, Emerman M, Hope TJ (2002) Visualization of the intracellular behavior of HIV in living cells. J Cell Biol 159: 441-452.

McNatt MW, Zang T, Hatziioannou T, Bartlett M, Fofana IB, Johnson WE, Neil SJ, Bieniasz PD (2009) Speciesspecific activity of HIV-1 Vpu and positive selection of tetherin transmembrane domain variants. PLoS Pathog 5: e1000300.

Meehan AM, Saenz DT, Morrison JH, Garcia-Rivera JA, Peretz M, Llano M, Poeschla EM (2009) LEDGF/p75 proteins with alternative chromatin tethers are functional HIV-1 cofactors. PLoS Pathog 5: e1000522.

Melikyan GB (2008) Common principles and intermediates of viral protein-mediated fusion: the HIV-1 paradigm. Retrovirology 5: 111.

Melikyan GB, Markosyan RM, Hemmati H, Delmedico MK, Lambert DM, Cohen FS (2000) Evidence that the transition of HIV-1 gp41 into a six-helix bundle, not the bundle configuration, induces membrane fusion. $J$ Cell Biol 151: 413-423.

Metharom P, Takyar S, Xia HH, Ellem KA, Macmillan J, Shepherd RW, Wilcox GE, Wei MQ (2000) Novel bovine lentiviral vectors based on Jembrana disease virus. J Gene Med 2: 176-185.

Miest T, Saenz D, Meehan A, Llano M, Poeschla EM (2009) Intensive RNAi with lentiviral vectors in mammalian cells. Methods 47: 298-303.

Miller MD, Farnet CM, Bushman FD (1997) Human immunodeficiency virus type 1 preintegration complexes: studies of organization and composition. J Virol 71: 5382-5390.

Millington M, Arndt A, Boyd M, Applegate T, Shen S (2009) Towards a clinically relevant lentiviral transduction protocol for primary human CD34 hematopoietic stem/progenitor cells. PLoS One 4: e6461.

Mitchell RS, Beitzel BF, Schroder AR, Shinn P, Chen H, Berry CC, Ecker JR, Bushman FD (2004) Retroviral 
DNA integration: ASLV, HIV, and MLV show distinct target site preferences. PLOS Biol 2: e234.

Mitta B, Rimann M, Ehrengruber MU, Ehrbar M, Djonov V, Kelm J, Fussenegger M (2002) Advanced modular self-inactivating lentiviral expression vectors for multigene interventions in mammalian cells and in vivo transduction. Nucleic Acids Res 30: e113.

Mitta B, Rimann M, Fussenegger M (2005) Detailed design and comparative analysis of protocols for optimized production of high-performance HIV-1-derived lentiviral particles. Metab Eng 7: 426-436.

Miyake K, Miyake N, Shimada T (2007) Development of targeted gene transfer into human primary $\mathrm{T}$ lymphocytes and macrophages using high-titer recombinant HIV vectors. J Biotechnol 129: 532-538.

Miyoshi H, Blomer U, Takahashi M, Gage FH, Verma IM (1998) Development of a self-inactivating lentivirus vector. J Virol 72: 8150-8157.

Miyoshi H, Smith KA, Mosier DE, Verma IM, Torbett BE (1999) Transduction of human CD34+ cells that mediate long-term engraftment of NOD/SCID mice by HIV vectors. Science 283: 682-686.

Mizuguchi $\mathrm{H}, \mathrm{Xu} \mathrm{Z}$, Ishii-Watabe A, Uchida E, Hayakawa $\mathrm{T}$ (2000) IRES-dependent second gene expression is significantly lower than cap-dependent first gene expression in a bicistronic vector. Mol Ther 1: 376-382.

Mochizuki H, Schwartz JP, Tanaka K, Brady RO, Reiser J (1998) High-titer human immunodeficiency virus type 1-based vector systems for gene delivery into nondividing cells. J Virol 72: 8873-8883.

Moffat J, Grueneberg DA, Yang X, Kim SY, Kloepfer AM, Hinkle G, Piqani B, Eisenhaure TM, Luo B, Grenier JK, Carpenter AE, Foo SY, Stewart SA, Stockwell BR, Hacohen N, Hahn WC, Lander ES, Sabatini DM, Root DE (2006) A lentiviral RNAi library for human and mouse genes applied to an arrayed viral high-content screen. Cell 124: 1283-1298.

Mok HP, Javed S, Lever A (2007) Stable gene expression occurs from a minority of integrated HIV-1-based vectors: transcriptional silencing is present in the majority. Gene Ther 14: 741-751.

Moldt B, Staunstrup NH, Jakobsen M, Yáñez-Muñoz RJ, Mikkelsen JG (2008) Genomic insertion of lentiviral DNA circles directed by the yeast Flp recombinase. BMC Biotechnol 8: 60.

Montagnier L, Dauguet C, Axler C, Chamaret S, Gruest J, Nugeyre MT, Rey F, Barre-Sinoussi F, Chermann JC (1984) A new type of retrovirus isolated from patients presenting with lymphadenopathy and acquired immune deficiency syndrome: structural and antigenic relatedness with equine infectious anaemia virus. Ann Virologie 135e: 119-134.

Montini E, Cesana D, Schmidt M, Sanvito F, Bartholomae CC, Ranzani M, Benedicenti F, Sergi LS, Ambrosi A, Ponzoni M, Doglioni C, Di Serio C, von Kalle C, Naldini L (2009) The genotoxic potential of retroviral vectors is strongly modulated by vector design and integration site selection in a mouse model of HSC gene therapy. $J$ Clin Invest 119: 964-975.

Moosmann P, Georgiev O, Thiesen HJ, Hagmann M, Schaffner W (1997) Silencing of RNA polymerases II and III-dependent transcription by the KRAB protein domain of KOX1, a Kruppel-type zinc finger factor. Biol Chem 378: 669-677.

Moreau-Gaudry F, Xia P, Jiang G, Perelman NP, Bauer G, Ellis J, Surinya KH, Mavilio F, Shen CK, Malik P (2001) High-level erythroid-specific gene expression in primary human and murine hematopoietic cells with self-inactivating lentiviral vectors. Blood 98: 2664-2672.
Morris KV, Rossi JJ (2006) Lentivirus-mediated RNA interference therapy for human immunodeficiency virus type 1 infection. Hum Gene Ther 17: 479-486.

Mougel M, Houzet L, Darlix JL (2009) When is it time for reverse transcription to start and go? Retrovirology 6: 24.

Mselli-Lakhal L, Favier C, Da Silva Teixeira MF, Chettab K, Legras C, Ronfort C, Verdier G, Mornex JF, Chebloune Y (1998) Defective RNA packaging is responsible for low transduction efficiency of CAEV-based vectors. Arch Virol 143: 681-695.

Murphy S (1997) Differential in vivo activation of the class II and class III snRNA genes by the POU-specific domain of Oct-1. Nucleic Acids Res 25: 2068-2076.

Nakajima T, Nakamaru K, Ido E, Terao K, Hayami M, Hasegawa M (2000) Development of novel simian immunodeficiency virus vectors carrying a dual gene expression system. Hum Gene Ther 11: 1863-1874.

Naldini L, Blomer U, Gage FH, Trono D, Verma IM (1996b) Efficient transfer, integration, and sustained long-term expression of the transgene in adult rat brains injected with a lentiviral vector. Proc Natl Acad Sci USA 93: 11382-11388.

Naldini L, Blomer U, Gallay P, Ory D, Mulligan R, Gage FH, Verma IM, Trono D (1996a) In vivo gene delivery and stable transduction of nondividing cells by a lentiviral vector. Science 272: 263-267.

Nanou A, Azzouz M (2009) Gene therapy for neurodegenerative diseases based on lentiviral vectors. Prog Brain Res 175: 187-200.

Nappi F, Chiozzini C, Bordignon V, Borsetti A, Bellino S, Cippitelli M, Barillari G, Caputo A, Tyagi M, Giacca M, Ensoli B (2009) Immobilized HIV-1 Tat protein promotes gene transfer via a transactivation-independent mechanism which requires binding of Tat to viral particles. J Gene Med 11: 955-965.

Negre D, Mangeot PE, Duisit G, Blanchard S, Vidalain PO, Leissner P, Winter AJ, Rabourdin-Combe C, Mehtali M, Moullier P, Darlix JL, Cosset FL (2000) Characterization of novel safe lentiviral vectors derived from simian immunodeficiency virus (SIVmac251) that efficiently transduce mature human dendritic cells. Gene Ther 7: 1613-1623.

Neil SJ, Zang T, Bieniasz PD (2008) Tetherin inhibits retrovirus release and is antagonized by HIV-1 Vpu. Nature 451: 425-430.

Neschadim A, McCart JA, Keating A, Medin JA (2007) A roadmap to safe, efficient, and stable lentivirus-mediated gene therapy with hematopoietic cell transplantation. Biol Blood Marrow Transplant 13: 1407-1416.

Ngumbela KC, Ryan KP, Sivamurthy R, Brockman MA, Gandhi RT, Bhardwaj N, Kavanagh DG (2008) Quantitative effect of suboptimal codon usage on translational efficiency of mRNA encoding HIV-1 gag in intact $\mathrm{T}$ cells. PLOS ONE 3: e2356.

Nguyen DG, Booth A, Gould SJ, Hildreth JE (2003) Evidence that HIV budding in primary macrophages occurs through the exosome release pathway. J Biol Chem 278: 52347-52354.

Nguyen DH, Hildreth JE (2000) Evidence for budding of human immunodeficiency virus type 1 selectively from glycolipid-enriched membrane lipid rafts. J Virol 74: 3264-3272.

Nguyen TH, Oberholzer J, Birraux J, Majno P, Morel P, Trono D (2002) Highly efficient lentiviral vector-mediated transduction of nondividing, fully reimplantable primary hepatocytes. Mol Ther 6: 199-209.

Ni Y, Sun S, Oparaocha I, Humeau L, Davis B, Cohen R, Binder G, Chang YN, Slepushkin V, Dropulic B (2005) 
Generation of a packaging cell line for prolonged largescale production of high-titer HIV-1-based lentiviral vector. J Gene Med 7: 818-834.

Nielsen TT, Jakobsson J, Rosenqvist N, Lundberg C (2009) Incorporating double copies of a chromatin insulator into lentiviral vectors results in less viral integrants. BMC Biotechnol 9: 13.

Nishizawa Y, Usukura J, Singh DP, Chylack LT Jr, Shinohara T (2001) Spatial and temporal dynamics of two alternatively spliced regulatory factors, lens epithelium-derived growth factor (ledgf/p75) and p52, in the nucleus. Cell Tissue Res 305: 107-114.

Nitahara-Kasahara $Y$, Kamata M, Yamamoto T, Zhang X, Miyamoto $Y$, Muneta K, Iijima S, Yoneda $Y$, Tsunetsugu-Yokota Y, Aida Y (2007) Novel nuclear import of Vpr promoted by importin alpha is crucial for human immunodeficiency virus type 1 replication in macrophages. J Virol 81: 5284-5293.

Nomaguchi M, Fujita M, Adachi A (2008) Role of HIV-1 $\mathrm{Vpu}$ protein for virus spread and pathogenesis. Microbes Infect 10: 960-967.

O'Doherty U, Swiggard WJ, Malim MH (2000) Human immunodeficiency virus type 1 spinoculation enhances infection through virus binding. J Virol 74: 10074-10080.

Oh T, Bajwa A, Jia G, Park F (2007) Lentiviral vector design using alternative RNA export elements. Retrovirology 4: 38.

Ohlmann T, Lopez-Lastra M, Darlix JL (2000) An internal ribosome entry segment promotes translation of the simian immunodeficiency virus genomic RNA. J Biol Chem 275: 11899-11906.

Okada Y, Ueshin Y, Hasuwa H, Takumi K, Okabe M, Ikawa M (2009) Targeted gene modification in mouse ES cells using integrase-defective lentiviral vectors. Genesis 47: 217-223.

Okumura A, Alce T, Lubyova B, Ezelle H, Strebel K, Pitha PM (2008) HIV-1 accessory proteins VPR and Vif modulate antiviral response by targeting IRF-3 for degradation. Virology 373: 85-97.

Olsen JC (1998) Gene transfer vectors derived from equine infectious anemia virus. Gene Ther 5: 1481-1487.

Ono A, Freed EO (2004) Cell-type-dependent targeting of human immunodeficiency virus type 1 assembly to the plasma membrane and the multivesicular body. J Virol 78: 1552-1563.

Ono A, Waheed AA, Freed EO (2007) Depletion of cellular cholesterol inhibits membrane binding and higher-order multimerization of human immunodeficiency virus type 1 Gag. Virology 360: 27-35.

Oppermann M (2004) Chemokine receptor CCR5: insights into structure, function, and regulation. Cell Signal 16: 1201-1210.

Ory DS, Neugeboren BA, Mulligan RC (1996) A stable human-derived packaging cell line for production of high titer retrovirus/vesicular stomatitis virus G pseudotypes. Proc Natl Acad Sci USA 93: 11400-11406.

Osti D, Marras E, Ceriani I, Grassini G, Rubino T, Vigano D, Parolaro D, Perletti G (2006) Comparative analysis of molecular strategies attenuating positional effects in lentiviral vectors carrying multiple genes. J Virol Methods 136: 93-101.

Ott DE (2008) Cellular proteins detected in HIV-1. Rev Med Virol 18: 159-175.

Ott MG, Schmidt M, Schwarzwaelder K, Stein S, Siler U, Koehl U, Glimm H, Kuhlcke K, Schilz A, Kunkel H, Naundorf S, Brinkmann A, Deichmann A, Fischer M, Ball C, Pilz I, Dunbar C, Du Y, Jenkins NA, Copeland NG, Luthi U, Hassan M, Thrasher AJ, Hoelzer D, von Kalle C, Seger R, Grez M (2006) Correction of X- linked chronic granulomatous disease by gene therapy, augmented by insertional activation of MDS1-EVI1, PRDM16 or SETBP1. Nat Med 12: 401-409.

Page KA, Landau NR, Littman DR (1990) Construction and use of a human immunodeficiency virus vector for analysis of virus infectivity. J Virol 64: 5270-5276.

Pandya S, Boris-Lawrie K, Leung NJ, Akkina R, Planelles V (2001) Development of an Rev-independent, minimal simian immunodeficiency virus-derived vector system. Hum Gene Ther 12: 847-857.

Pante N, Kann M (2002) Nuclear pore complex is able to transport macromolecules with diameters of about 39 nm. Mol Biol Cell 13: 425-434.

Parissi V, Calmels C, De Soultrait VR, Caumont A, Fournier M, Chaignepain S, Litvak S (2001) Functional interactions of human immunodeficiency virus type 1 integrase with human and yeast HSP60. J Virol 75: 11344-11353.

Park F (2007) Lentiviral vectors: are they the future of animal transgenesis? Physiol Genomics 31: 159-173.

Park F, Kay MA (2001) Modified HIV-1 based lentiviral vectors have an effect on viral transduction efficiency and gene expression in vitro and in vivo. Mol Ther 4: 164-173.

Park IW, He JJ (2009) HIV-1 Nef-mediated inhibition of T cell migration and its molecular determinants. J Leukoc Biol 86: 1171-1178.

Parolin C, Dorfman T, Palu G, Gottlinger H, Sodroski J (1994) Analysis in human immunodeficiency virus type 1 vectors of cis-acting sequences that affect gene transfer into human lymphocytes. J Virol 68: 3888-3895.

Paxton W, Connor RI, Landau NR (1993) Incorporation of Vpr into human immunodeficiency virus type 1 virions: requirement for the p6 region of gag and mutational analysis. J Virol 67: 7229-7237.

Pearson R, Kim YK, Hokello J, Lassen K, Friedman J, Tyagi M, Karn J (2008) Epigenetic silencing of human immunodeficiency virus (HIV) transcription by formation of restrictive chromatin structures at the viral long terminal repeat drives the progressive entry of HIV into latency. J Virol 82: 12291-12303.

Pery E, Rajendran KS, Brazier AJ, Gabuzda D (2009) Regulation of APOBEC3 proteins by a novel YXXL motif in human immunodeficiency virus type 1 Vif and simian immunodeficiency virus SIVagm Vif. J Virol 83: 23742381.

Pessler F, Cron RQ (2004) Reciprocal regulation of the nuclear factor of activated T cells and HIV-1. Genes Immun 5: 158-167.

Pfeifer A, Kessler T, Yang M, Baranov E, Kootstra N, Cheresh DA, Hoffman RM, Verma IM (2001) Transduction of liver cells by lentiviral vectors: analysis in living animals by fluorescence imaging. Mol Ther 3: 319-322.

Pfeifer A, Hofmann A (2009) Lentiviral transgenesis. Methods Mol Biol 530: 391-405.

Philippe S, Sarkis C, Barkats M, Mammeri H, Ladroue C, Petit C, Mallet J, Serguera C (2006) Lentiviral vectors with a defective integrase allow efficient and sustained transgene expression in vitro and in vivo. Proc Natl Acad Sci USA 103: 17684-17689.

Piacentini L, Biasin M, Fenizia C, Clerici M (2009) Genetic correlates of protection against HIV infection: the ally within. J Intern Med 265: 110-124.

Pistello M, Vannucci L, Ravani A, Bonci F, Chiuppesi F, Del Santo B, Freer G, Bendinelli M (2007) Streamlined design of a self-inactivating feline immunodeficiency virus vector for transducing ex vivo dendritic cells and T lymphocytes. Genet Vaccines Ther 5: 8. 
Pizzato M, Popova E, Gottlinger HG (2008) Nef can enhance the infectivity of receptor-pseudotyped human immunodeficiency virus type 1 particles. J Virol 82: 10811-10819.

Plantier JC, Leoz M, Dickerson JE, De Oliveira F, Cordonnier F, Lemee $V$, Damond F, Robertson DL, Simon F (2009) A new human immunodeficiency virus derived from gorillas. Nat Med 15: 871-872.

Pluta K, Diehl W, Zhang XY, Kutner R, Bialkowska A, Reiser J (2007) Lentiviral vectors encoding tetracyclinedependent repressors and transactivators for reversible knockdown of gene expression: a comparative study. BMC Biotechnol 7: 41.

Pluta K, Luce MJ, Bao L, Agha-Mohammadi S, Reiser J (2005) Tight control of transgene expression by lentivirus vectors containing second-generation tetracyclineresponsive promoters. J Gene Med 7: 803-817.

Poeschla E, Corbeau P, Wong-Staal F (1996) Development of HIV vectors for anti-HIV gene therapy. Proc Natl Acad Sci USA 93: 11395-11399.

Poeschla E, Gilbert J, Li X, Huang S, Ho A, Wong-Staal F (1998a) Identification of a human immunodeficiency virus type 2 (HIV-2) encapsidation determinant and transduction of nondividing human cells by HIV-2based lentivirus vectors. J Virol 72: 6527-6536.

Poeschla EM (2008) Integrase, LEDGF/p75 and HIV replication. Cell Mol Life Sci 65: 1403-1424.

Poeschla EM, Wong-Staal F, Looney DJ (1998b) Efficient transduction of nondividing human cells by feline immunodeficiency virus lentiviral vectors. Nat Med 4: 354-357.

Pollard VW, Malim MH (1998) The HIV-1 Rev protein. Annu Rev Microbiol 52: 491-532.

Pond SJ, Ridgeway WK, Robertson R, Wang J, Millar DP (2009) HIV-1 Rev protein assembles on viral RNA one molecule at a time. Proc Natl Acad Sci USA 106: 14041408.

Popov S, Rexach M, Zybarth G, Reiling N, Lee MA, Ratner L, Lane CM, Moore MS, Blobel G, Bukrinsky M (1998) Viral protein $\mathrm{R}$ regulates nuclear import of the HIV-1 pre-integration complex. EMBO J 17: 909-917.

Poznansky M, Lever A, Bergeron L, Haseltine W, Sodroski J (1991) Gene transfer into human lymphocytes by a defective human immunodeficiency virus type 1 vector. J Virol 65: 532-536.

Ptak RG, Fu W, Sanders-Beer BE, Dickerson JE, Pinney JW, Robertson DL, Rozanov MN, Katz KS, Maglott DR, Pruitt KD, Dieffenbach CW (2008) Cataloguing the HIV type 1 human protein interaction network. AIDS Res Hum Retroviruses 24: 1497-1502.

Puigdomenech I, Massanella M, Izquierdo-Useros N, RuizHernandez R, Curriu M, Bofill M, Martinez-Picado J, Juan M, Clotet B, Blanco J (2008) HIV transfer between CD4 T cells does not require LFA-1 binding to ICAM1 and is governed by the interaction of HIV envelope glycoprotein with CD4. Retrovirology 5: 32.

Purcell DF, Martin MA (1993) Alternative splicing of human immunodeficiency virus type 1 mRNA modulates viral protein expression, replication, and infectivity. J Virol 67: 6365-6378.

Rahim AA, Wong AM, Howe SJ, Buckley SM, Acosta-Saltos AD, Elston KE, Ward NJ, Philpott NJ, Cooper JD, Anderson PN, Waddington SN, Thrasher AJ, Raivich G (2009) Efficient gene delivery to the adult and fetal CNS using pseudotyped non-integrating lentiviral vectors. Gene Ther 16: 509-520.

Ralph GS, Binley K, Wong LF, Azzouz M, Mazarakis ND (2006) Gene therapy for neurodegenerative and ocular diseases using lentiviral vectors. Clin Sci (Lond) 110: 37-46.

Ramezani A, Hawley TS, Hawley RG (2003) Performanceand safety-enhanced lentiviral vectors containing the human interferon-beta scaffold attachment region and the chicken beta-globin insulator. Blood 101: 4717-4724.

Ramezani A, Hawley TS, Hawley RG (2008) Combinatorial incorporation of enhancer-blocking components of the chicken beta-globin 5'HS4 and human T-cell receptor alpha/delta BEAD-1 insulators in self-inactivating retroviral vectors reduces their genotoxic potential. Stem Cells 26: 3257-3266.

Rang A, Will H (2000) The tetracycline-responsive promoter contains functional interferon-inducible response elements. Nucleic Acids Res 28: 1120-1125.

Regulier E, Pereira de Almeida L, Sommer B, Aebischer P, Deglon N (2002) Dose-dependent neuroprotective effect of ciliary neurotrophic factor delivered via tetracycline-regulated lentiviral vectors in the quinolinic acid rat model of Huntington's disease. Hum Gene Ther 13: 1981-1990.

Regulier E, Trottier Y, Perrin V, Aebischer P, Deglon N (2003) Early and reversible neuropathology induced by tetracycline-regulated lentiviral overexpression of mutant huntingtin in rat striatum. Hum Mol Genet 12: 2827-2836.

Reiser J (2000) Production and concentration of pseudotyped HIV-1-based gene transfer vectors. Gene Ther 7: 910-913.

Reiser J, Harmison G, Kluepfel-Stahl S, Brady RO, Karlsson S, Schubert M (1996) Transduction of nondividing cells using pseudotyped defective high-titer HIV type 1 particles. Proc Natl Acad Sci USA 93: 15266-15271.

Reiser J, Lai Z, Zhang XY, Brady RO (2000) Development of multigene and regulated lentivirus vectors. J Virol 74: 10589-10599.

Resh MD (2005) Intracellular trafficking of HIV-1 Gag: how Gag interacts with cell membranes and makes viral particles. AIDS Rev 7: 84-91.

Rhodes T, Wargo H, Hu WS (2003) High rates of human immunodeficiency virus type 1 recombination: nearrandom segregation of markers one kilobase apart in one round of viral replication. J Virol 77: 11193-11200.

Richard E, Robert E, Cario-Andre M, Ged C, Geronimi F, Gerson SL, de Verneuil H, Moreau-Gaudry F (2004) Hematopoietic stem cell gene therapy of murine protoporphyria by methylguanine-DNA-methyltransferase-mediated in vivo drug selection. Gene Ther 11: 1638-1647.

Richman DD, Margolis DM, Delaney M, Greene WC, Hazuda D, Pomerantz RJ (2009) The challenge of finding a cure for HIV infection. Science 323: 1304-1307.

Ricks DM, Kutner R, Zhang XY, Welsh DA, Reiser J (2008) Optimized lentiviral transduction of mouse bone marrow-derived mesenchymal stem cells. Stem Cells Dev 17: 441-450.

Rizvi TA, Lew KA, Murphy EC Jr, Schmidt RD (1996) Role of Mason-Pfizer monkey virus (MPMV) constitutive transport element (CTE) in the propagation of MPMV vectors by genetic complementation using homologous/ heterologous env genes. Virology 224: 517-532.

Roe T, Reynolds TC, Yu G, Brown PO (1993) Integration of murine leukemia virus DNA depends on mitosis. EMBO J 12: 2099-2108.

Root DE, Hacohen N, Hahn WC, Lander ES, Sabatini DM (2006) Genome-scale loss-of-function screening with a lentiviral RNAi library. Nat Methods 3: 715-719.

Rosenbluh J, Hayouka Z, Loya S, Levin A, Armon-Omer A, Britan E, Hizi A, Kotler M, Friedler A, Loyter A (2007) 
Interaction between HIV-1 Rev and integrase proteins: a basis for the development of anti-HIV peptides. J Biol Chem 282: 15743-15753.

Rougemaille M, Villa T, Gudipati RK, Libri D (2008) mRNA journey to the cytoplasm: attire required. Biol Cell 100: 327-342.

Russell RS, Liang C, Wainberg MA (2004) Is HIV-1 RNA dimerization a prerequisite for packaging? Yes, no, probably? Retrovirology 1: 23.

Ryan RF, Schultz DC, Ayyanathan K, Singh PB, Friedman JR, Fredericks WJ, Rauscher FJ 3rd (1999) KAP-1 corepressor protein interacts and colocalizes with heterochromatic and euchromatic HP1 proteins: a potential role for Kruppel-associated box-zinc finger proteins in heterochromatin-mediated gene silencing. Mol Cell Biol 19: 4366-4378.

Saad JS, Miller J, Tai J, Kim A, Ghanam RH, Summers MF (2006) Structural basis for targeting HIV-1 Gag proteins to the plasma membrane for virus assembly. Proc Natl Acad Sci USA 103: 11364-11369.

Saadatmand J, Guo F, Cen S, Niu M, Kleiman L (2008) Interactions of reverse transcriptase sequences in Pol with Gag and LysRS in the HIV-1 tRNALys3 packaging/annealing complex. Virology 380: 109-117.

Sachdeva G, D'Costa J, Cho JE, Kachapati K, Choudhry V, Arya SK (2007) Chimeric HIV-1 and HIV-2 lentiviral vectors with added safety insurance. J Med Virol 79: 118-126.

Salgado GF, Marquant R, Vogel A, Alves ID, Feller SE, Morellet N, Bouaziz S (2009) Structural studies of HIV1 Gag p6ct and its interaction with Vpr determined by solution nuclear magnetic resonance (dagger) (,) (double dagger) Biochemistry 48: 2355-2367.

Salmon P, Kindler V, Ducrey O, Chapuis B, Zubler RH, Trono D (2000a) High-level transgene expression in human hematopoietic progenitors and differentiated blood lineages after transduction with improved lentiviral vectors. Blood 96: 3392-3398.

Salmon P, Oberholzer J, Occhiodoro T, Morel P, Lou J, Trono D (2000b) Reversible immortalization of human primary cells by lentivector-mediated transfer of specific genes. Mol Ther 2: 404-414.

Sangiolo D, Lesnikova M, Nash RA, Jensen MC, Nikitine A, Kiem HP, Georges GE (2007) Lentiviral vector conferring resistance to mycophenolate mofetil and sensitivity to ganciclovir for in vivo T-cell selection. Gene Ther 14: 1549-1554.

Santoni de Sio F, Naldini L (2009) Short-term culture of human CD34+ cells for lentiviral gene transfer. Methods Mol Biol 506: 59-70.

Saphire AC, Gallay PA, Bark SJ (2006) Proteomic analysis of human immunodeficiency virus using liquid chromatography/tandem mass spectrometry effectively distinguishes specific incorporated host proteins. J Proteome Res 5: 530-538.

Sarkis C, Philippe S, Mallet J, Serguera C (2008) Non-integrating lentiviral vectors. Curr Gene Ther 8: 430-437.

Sastry L, Johnson T, Hobson MJ, Smucker B, Cornetta K (2002) Titering lentiviral vectors: comparison of DNA, RNA and marker expression methods. Gene Ther 9: 1155-1162.

Sastry L, Xu Y, Cooper R, Pollok K, Cornetta K (2004) Evaluation of plasmid DNA removal from lentiviral vectors by benzonase treatment. Hum Gene Ther 15: 221-226.

Sastry L, Cornetta K (2009) Detection of replication competent retrovirus and lentivirus. Methods Mol Biol 506: 243-263.

Sato H, Orenstein J, Dimitrov D, Martin M (1992) Cell-tocell spread of HIV-1 occurs within minutes and may not involve the participation of virus particles. Virology 186: 712-724.

Sattentau QJ, Moore JP (1991) Conformational changes induced in the human immunodeficiency virus envelope glycoprotein by soluble CD4 binding. J Exp Med 174: 407-415.

Scarlata S, Carter C (2003) Role of HIV-1 Gag domains in viral assembly. Biochim Biophys Acta 1614: 62-72.

Schambach A, Bohne J, Baum C, Hermann FG, Egerer L, von Laer D, Giroglou T (2006) Woodchuck hepatitis virus post-transcriptional regulatory element deleted from $X$ protein and promoter sequences enhances retroviral vector titer and expression. Gene Ther 13: 641645.

Schambach A, Galla M, Maetzig T, Loew R, Baum C (2007) Improving transcriptional termination of self-inactivating gamma-retroviral and lentiviral vectors. Mol Ther 15: 1167-1173.

Scherr M, Eder M (2007) Gene silencing by small regulatory RNAs in mammalian cells. Cell Cycle 6: 444-449.

Schmid M, Jensen TH (2008) Quality control of mRNP in the nucleus. Chromosoma 117: 419-429.

Schroder AR, Shinn P, Chen H, Berry C, Ecker JR, Bushman F (2002) HIV-1 integration in the human genome favors active genes and local hotspots. Cell 110: 521529.

Schrofelbauer B, Senger T, Manning G, Landau NR (2006) Mutational alteration of human immunodeficiency virus type 1 Vif allows for functional interaction with nonhuman primate APOBEC3G. J Virol 80: 5984-5991.

Schwartz S, Felber BK, Benko DM, Fenyo EM, Pavlakis GN (1990) Cloning and functional analysis of multiply spliced mRNA species of human immunodeficiency virus type 1. J Virol 64: 2519-2529.

Schwartz S, Felber BK, Pavlakis GN (1991) Expression of human immunodeficiency virus type 1 vif and vpr mRNAs is rev-dependent and regulated by splicing. Virology 183: 677-686.

Schwartz S, Felber BK, Pavlakis GN (1992) Mechanism of translation of monocistronic and multicistronic human immunodeficiency virus type 1 mRNAs. Mol Cell Biol 12: 207-219.

Schweighardt B, Roy AM, Meiklejohn DA, Grace EJ 2nd, Moretto WJ, Heymann JJ, Nixon DF (2004) R5 human immunodeficiency virus type 1 (HIV-1) replicates more efficiently in primary CD4+ T-cell cultures than $\mathrm{X} 4$ HIV-1. J Virol 78: 9164-9173.

Seelamgari A, Maddukuri A, Berro R, de la Fuente C, Kehn K, Deng L, Dadgar S, Bottazzi ME, Ghedin E, Pumfery A, Kashanchi F (2004) Role of viral regulatory and accessory proteins in HIV-1 replication. Front Biosci 9: 2388-2413.

Segura MM, Garnier A, Durocher Y, Coelho H, Kamen A (2007) Production of lentiviral vectors by large-scale transient transfection of suspension cultures and affinity chromatography purification. Biotechnol Bioeng 98: 789-799.

Segura MM, Kamen A, Garnier A (2006) Downstream processing of oncoretroviral and lentiviral gene therapy vectors. Biotechnol Adv 24: 321-337.

Semple-Rowland SL, Eccles KS, Humberstone EJ (2007) Targeted expression of two proteins in neural retina using self-inactivating, insulated lentiviral vectors carrying two internal independent promoters. Mol Vis 13: 2001-2011.

Sena-Esteves M, Tebbets JC, Steffens S, Crombleholme T, Flake AW (2004) Optimized large-scale production of high titer lentivirus vector pseudotypes. J Virol Methods 122: 131-139. 
Senatore B, Cafieri A, Di Marino I, Rosati M, Di Nocera PP, Grimaldi G (1999) A variety of RNA polymerases II and III-dependent promoter classes is repressed by factors containing the Kruppel-associated/finger preceding box of zinc finger proteins. Gene 234: 381-394.

Shao L, Feng W, Sun Y, Bai H, Liu J, Currie C, Kim J, Gama R, Wang Z, Qian Z, Liaw L, Wu WS (2009) Generation of iPS cells using defined factors linked via the self-cleaving $2 \mathrm{~A}$ sequences in a single open reading frame. Cell Res 19: 296-306.

Shaw KL, Pais E, Ge S, Hardee C, Skelton D, Hollis RP, Crooks GM, Kohn DB (2009) Lentiviral vectors with amplified beta cell-specific gene expression. Gene Ther 16: $998-1008$.

Sheehy AM, Gaddis NC, Choi JD, Malim MH (2002) Isolation of a human gene that inhibits HIV-1 infection and is suppressed by the viral Vif protein. Nature 418: 646-650.

Shehu-Xhilaga M, Crowe SM, Mak J (2001) Maintenance of the Gag/Gag-Pol ratio is important for human immunodeficiency virus type 1 RNA dimerization and viral infectivity. J Virol 75: 1834-1841.

Shin KJ, Wall EA, Zavzavadjian JR, Santat LA, Liu J, Hwang JI, Rebres R, Roach T, Seaman W, Simon MI, Fraser ID (2006) A single lentiviral vector platform for microRNA-based conditional RNA interference and coordinated transgene expression. Proc Natl Acad Sci USA 103: 13759-13764.

Shukla V, Coumoul X, Deng CX (2007) RNAi-based conditional gene knockdown in mice using a U6 promoter driven vector. Int J Biol Sci 3: 91-99.

Shun MC, Botbol Y, Li X, Di Nunzio F, Daigle JE, Yan N, Lieberman J, Lavigne M, Engelman A (2008) Identification and characterization of PWWP domain residues critical for LEDGF/p75 chromatin binding and human immunodeficiency virus type 1 infectivity. J Virol 82: 11555-11567.

Shun MC, Raghavendra NK, Vandegraaff N, Daigle JE, Hughes S, Kellam P, Cherepanov P, Engelman A (2007) LEDGF/p75 functions downstream from preintegration complex formation to effect gene-specific HIV-1 integration. Genes Dev 21: 1767-1778.

Signoret N, Hewlett L, Wavre S, Pelchen-Matthews A, Oppermann M, Marsh M (2005) Agonist-induced endocytosis of CC chemokine receptor 5 is clathrin dependent. Mol Biol Cell 16: 902-917.

Simon JH, Miller DL, Fouchier RA, Soares MA, Peden KW, Malim MH (1998) The regulation of primate immunodeficiency virus infectivity by Vif is cell species restricted: a role for Vif in determining virus host range and cross-species transmission. EMBO J 17: 1259-1267.

Singer O, Verma IM (2008) Applications of lentiviral vectors for shRNA delivery and transgenesis. Curr Gene Ther 8: 483-488.

Singh DP, Kimura A, Chylack LT Jr, Shinohara T (2000) Lens epithelium-derived growth factor (LEDGF/p75) and p52 are derived from a single gene by alternative splicing. Gene 242: 265-273.

Sirois M, Robitaille L, Sasik R, Estaquier J, Fortin J, Corbeil J (2008) R5 and X4 HIV viruses differentially modulate host gene expression in resting CD4+ T cells. AIDS Res Hum Retroviruses 24: 485-493.

Sirven A, Pflumio F, Zennou V, Titeux M, Vainchenker W, Coulombel L, Dubart-Kupperschmitt A, Charneau P (2000) The human immunodeficiency virus type-1 central DNA flap is a crucial determinant for lentiviral vector nuclear import and gene transduction of human hematopoietic stem cells. Blood 96: 4103-4110.
Skasko M, Kim B (2008) Compensatory role of human immunodeficiency virus central polypurine tract sequence in kinetically disrupted reverse transcription. J Virol 82: 7716-7720.

Slepushkin V, Chang N, Cohen R (2003) Large-scale purification of a lentiviral vector by size exclusion chromatography or Mustang Q ion exchange capsule. Bioprocess J 2: 89-95.

Smith AJ, Srinivasakumar N, Hammarskjold ML, Rekosh D (1993) Requirements for incorporation of Pr160gagpol from human immunodeficiency virus type 1 into virus-like particles. J Virol 67: 2266-2275.

Sodora DL, Allan JS, Apetrei C, Brenchley JM, Douek DC, Else JG, Estes JD, Hahn BH, Hirsch VM, Kaur A, Kirchhoff F, Muller-Trutwin M, Pandrea I, Schmitz JE, Silvestri G (2009) Toward an AIDS vaccine: lessons from natural simian immunodeficiency virus infections of African nonhuman primate hosts. Nat Med 15: 861-865.

Sommer CA, Stadtfeld M, Murphy GJ, Hochedlinger K, Kotton DN, Mostoslavsky G (2009) Induced pluripotent stem cell generation using a single lentiviral stem cell cassette. Stem Cells 27: 543-549.

Soneoka Y, Cannon PM, Ramsdale EE, Griffiths JC, Romano G, Kingsman SM, Kingsman AJ (1995) A transient three-plasmid expression system for the production of high titer retroviral vectors. Nucleic Acids Res 23: 628-633.

Song R, Kafaie J, Yang L, Laughrea M (2007) HIV-1 viral RNA is selected in the form of monomers that dimerize in a three-step protease-dependent process; the DIS of stem-loop 1 initiates viral RNA dimerization. J Mol Biol 371: 1084-1098.

Sonigo P, Alizon M, Staskus K, Klatzmann D, Cole S, Danos O, Retzel E, Tiollais P, Haase A, Wain-Hobson S (1985) Nucleotide sequence of the visna lentivirus: relationship to the AIDS virus. Cell 42: 369-382.

Sowinski S, Jolly C, Berninghausen O, Purbhoo MA, Chauveau A, Kohler K, Oddos S, Eissmann P, Brodsky FM, Hopkins C, Onfelt B, Sattentau Q, Davis DM (2008) Membrane nanotubes physically connect $\mathrm{T}$ cells over long distances presenting a novel route for HIV-1 transmission. Nat Cell Biol 10: 211-219.

Spirin PV, Vil'gelm AE, Prasolov VS (2008) [Lentiviral vectors]. Mol Biol (Mosk) 42: 913-926 (in Russian).

Srinivasakumar N (2001) HIV-1 vector systems. Somat Cell Mol Genet 26: 51-81.

Srinivasakumar N, Chazal N, Helga-Maria C, Prasad S, Hammarskjold ML, Rekosh D (1997) The effect of viral regulatory protein expression on gene delivery by human immunodeficiency virus type 1 vectors produced in stable packaging cell lines. J Virol 71: 5841-5848.

Srinivasakumar N, Hammarskjold ML, Rekosh D (1995) Characterization of deletion mutations in the capsid region of human immunodeficiency virus type 1 that affect particle formation and Gag-Pol precursor incorporation. J Virol 69: 6106-6114.

Staunstrup NH, Moldt B, Mátés L, Villesen P, Jakobsen M, Ivics Z, Izsvák Z, Mikkelsen JG (2009) Hybrid lentivirus-transposon vectors with a random integration profile in human cells. Mol Ther 17: 1205-1214.

Stegmeier F, Hu G, Rickles RJ, Hannon GJ, Elledge SJ (2005) A lentiviral microRNA-based system for single-copy polymerase II-regulated RNA interference in mammalian cells. Proc Natl Acad Sci USA 102: 1321213217.

Stern P, Astrof S, Erkeland SJ, Schustak J, Sharp PA, Hynes RO (2008) A system for Cre-regulated RNA interference in vivo. Proc Natl Acad Sci USA 105: 13895-13900. 
Steuber-Buchberger P, Wurst W, Kuhn R (2008) Simultaneous Cre-mediated conditional knockdown of two genes in mice. Genesis 46: 144-151.

Stoltzfus CM, Madsen JM (2006) Role of viral splicing elements and cellular RNA binding proteins in regulation of HIV-1 alternative RNA splicing. Curr HIV Res 4: 43-55.

Strang BL, Ikeda Y, Cosset FL, Collins MK, Takeuchi Y (2004) Characterization of HIV-1 vectors with gammaretrovirus envelope glycoproteins produced from stable packaging cells. Gene Ther 11: 591-598.

Stripecke R, Cardoso AA, Pepper KA, Skelton DC, Yu XJ, Mascarenhas L, Weinberg KI, Nadler LM, Kohn DB (2000) Lentiviral vectors for efficient delivery of CD80 and granulocyte-macrophage- colony-stimulating factor in human acute lymphoblastic leukemia and acute myeloid leukemia cells to induce antileukemic immune responses. Blood 96: 1317-1326.

Strom AC, Forsberg M, Lillhager P, Westin G (1996) The transcription factors $\mathrm{Sp} 1$ and Oct-1 interact physically to regulate human U2 snRNA gene expression. Nucleic Acids Res 24: 1981-1986.

Stumptner-Cuvelette P, Morchoisne S, Dugast M, Le Gall S, Raposo G, Schwartz O, Benaroch P (2001) HIV-1 Nef impairs MHC class II antigen presentation and surface expression. Proc Natl Acad Sci USA 98: 12144-12149.

Su YR, Blakemore JL, Zhang Y, Linton MF, Fazio S (2008) Lentiviral transduction of apoAI into hematopoietic progenitor cells and macrophages: applications to cell therapy of atherosclerosis. Arterioscler Thromb Vasc Biol 28: 1439-1446.

Suhasini M, Reddy TR (2009) Cellular proteins and HIV-1 Rev function. Curr HIV Res 7: 91-100.

Sun J, Li D, Hao Y, Zhang Y, Fan W, Fu J, Hu Y, Liu Y, Shao Y (2009) Posttranscriptional regulatory elements enhance antigen expression and DNA vaccine efficacy. DNA Cell Biol 28: 233-240.

Swanson AK, Stoltzfus CM (1998) Overlapping cis sites used for splicing of HIV-1 env/nef and rev mRNAs. J Biol Chem 273: 34551-34557.

Swartz JE, Bor YC, Misawa Y, Rekosh D, Hammarskjold ML (2007) The shuttling SR protein 9G8 plays a role in translation of unspliced mRNA containing a constitutive transport element. J Biol Chem 282: 19844-19853.

Szulc J, Wiznerowicz M, Sauvain MO, Trono D, Aebischer $P$ (2006) A versatile tool for conditional gene expression and knockdown. Nat Methods 3: 109-116.

Szymczak AL, Vignali DA (2005) Development of 2A peptide-based strategies in the design of multicistronic vectors. Expert Opin Biol Ther 5: 627-638.

Takahashi K, Yamanaka S (2006) Induction of pluripotent stem cells from mouse embryonic and adult fibroblast cultures by defined factors. Cell 126: 663-676.

Takeuchi H, Matano T (2008) Host factors involved in resistance to retroviral infection. Microbiol Immunol 52: 318-325.

Tan SL, Parker PJ (2003) Emerging and diverse roles of protein kinase $\mathrm{C}$ in immune cell signalling. Biochem $\mathrm{J}$ 376: 545-552.

Tian J, Andreadis ST (2009) Independent and high-level dual-gene expression in adult stem-progenitor cells from a single lentiviral vector. Gene Ther 16: 874-884.

Tiscornia G, Tergaonkar V, Galimi F, Verma IM (2004) CRE recombinase-inducible RNA interference mediated by lentiviral vectors. Proc Natl Acad Sci USA 101: 7347-7351.

Toniatti C, Bujard H, Cortese R, Ciliberto G (2004) Gene therapy progress and prospects: transcription regulatory systems. Gene Ther 11: 649-657.
Towers GJ (2007) The control of viral infection by tripartite motif proteins and cyclophilin A. Retrovirology 4: 40.

Tsukahara T, Agawa H, Matsumoto S, Matsuda M, Ueno S, Yamashita Y, Yamada K, Tanaka N, Kojima K, Takeshita T (2006) Murine leukemia virus vector integration favors promoter regions and regional hot spots in a human T-cell line. Biochem Biophys Res Commun 345: 1099-1107.

Urbinati F, Arumugam P, Higashimoto T, Perumbeti A, Mitts K, Xia P, Malik P (2009) Mechanism of reduction in titers from lentivirus vectors carrying large inserts in the 3'LTR. Mol Ther 17: 1527-1536.

Urlinger S, Baron U, Thellmann M, Hasan MT, Bujard H, Hillen W (2000) Exploring the sequence space for tetracycline-dependent transcriptional activators: novel mutations yield expanded range and sensitivity. Proc Natl Acad Sci USA 97: 7963-7968.

Valente ST, Gilmartin GM, Mott C, Falkard B, Goff SP (2009) Inhibition of HIV-1 replication by eIF3f. Proc Natl Acad Sci USA 106: 4071-4078.

Valente ST, Goff SP (2009) Somatic cell genetic analyses to identify HIV-1 host restriction factors. Methods Mol Biol 485: 235-255.

van Hooijdonk LW, Ichwan M, Dijkmans TF, Schouten TG, de Backer MW, Adan RA, Verbeek FJ, Vreugdenhil E, Fitzsimons CP (2009) Lentivirus-mediated transgene delivery to the hippocampus reveals sub-field specific differences in expression. BMC Neurosci 10: 2.

Van Maele B, De Rijck J, De Clercq E, Debyser Z (2003) Impact of the central polypurine tract on the kinetics of human immunodeficiency virus type 1 vector transduction. J Virol 77: 4685-4694.

Vandegraaff N, Engelman A (2007) Molecular mechanisms of HIV integration and therapeutic intervention. Expert Rev Mol Med 9: 1-19.

Vatakis DN, Kim S, Kim N, Chow SA, Zack JA (2009) Human immunodeficiency virus integration efficiency and site selection in quiescent CD4+ T cells. J Virol 83: 6222-6233.

Veljkovic V, Veljkovic N, Glisic S, Ho MW (2008) AIDS vaccine: efficacy, safety and ethics. Vaccine 26: 30723077.

Ventura A, Meissner A, Dillon CP, McManus M, Sharp PA, Van Parijs L, Jaenisch R, Jacks T (2004) Cre-lox-regulated conditional RNA interference from transgenes. Proc Natl Acad Sci USA 101: 10380-10385.

Verhoeyen E, Cosset FL (2004) Surface-engineering of lentiviral vectors. J Gene Med 6 (Suppl 1): S83-S94.

Veron P, Boutin S, Martin S, Chaperot L, Plumas J, Davoust J, Masurier C (2009) Highly efficient transduction of human plasmacytoid dendritic cells without phenotypic and functional maturation. J Transl Med 7: 10.

Vieyra DS, Goodell MA (2007) Pluripotentiality and conditional transgene regulation in human embryonic stem cells expressing insulated tetracycline-ON transactivator. Stem Cells 25: 2559-2566.

Vigna E, Cavalieri S, Ailles L, Geuna M, Loew R, Bujard H, Naldini L (2002) Robust and efficient regulation of transgene expression in vivo by improved tetracyclinedependent lentiviral vectors. Mol Ther 5: 252-261.

Vink CA, Gaspar HB, Gabriel R, Schmidt M, McIvor RS, Thrasher AJ, Qasim W (2009) Sleeping beauty transposition from nonintegrating lentivirus. Mol Ther 17: 1197-1204.

Waheed AA, Freed EO (2009) Lipids and membrane microdomains in HIV-1 Replication. Virus Res 143: 162-176.

Wainberg MA, Jeang KT (2008) 25 years of HIV-1 research - progress and perspectives. BMC Med 6: 31. 
Wang GP, Levine BL, Binder GK, Berry CC, Malani N, McGarrity G, Tebas P, June CH, Bushman FD (2009a) Analysis of lentiviral vector integration in HIV+ study subjects receiving autologous infusions of gene modified CD4+ T cells. Mol Ther 17: 844-850.

Wang JH, Kwas C, Wu L (2009b) Intercellular adhesion molecule 1 (ICAM-1), but not ICAM-2 and -3, is important for dendritic cell-mediated human immunodeficiency virus type 1 transmission. J Virol 83: 4195-4204.

Wang Y, Iyer M, Annala AJ, Chappell S, Mauro V, Gambhir SS (2005) Noninvasive monitoring of target gene expression by imaging reporter gene expression in living animals using improved bicistronic vectors. $\mathrm{J} \mathrm{Nucl}$ Med 46: 667-674.

Wanisch K, Yáñez-Muñoz RJ (2009) Integration-deficient lentiviral vectors: a slow coming of age. Mol Ther 17: 1316-1332.

Watanabe S, Temin HM (1983) Construction of a helper cell line for avian reticuloendotheliosis virus cloning vectors. Mol Cell Biol 3: 2241-2249.

Watts JM, Dang KK, Gorelick RJ, Leonard CW, Bess JW Jr, Swanstrom R, Burch CL, Weeks KM (2009) Architecture and secondary structure of an entire HIV-1 RNA genome. Nature 460: 711-716.

Wei P, Garber ME, Fang SM, Fischer WH, Jones KA (1998) A novel CDK9-associated C-type cyclin interacts directly with HIV-1 Tat and mediates its high-affinity, loop-specific binding to TAR RNA. Cell 92: 451-462.

Weinberg JB, Matthews TJ, Cullen BR, Malim MH (1991) Productive human immunodeficiency virus type 1 (HIV-1) infection of nonproliferating human monocytes. J Exp Med 174: 1477-1482.

Weiss RA (2008) Special anniversary review: twenty-five years of human immunodeficiency virus research: successes and challenges. Clin Exp Immunol 152: 201-210.

Weissenhorn W, Hinz A, Gaudin Y (2007) Virus membrane fusion. FEBS Lett 581: 2150-2155.

Wheeler JX, Jones C, Thorpe R, Zhao Y (2007) Proteomics analysis of cellular components in lentiviral vector production using Gel-LC-MS/MS. Proteomics Clin Appl 1: 224-230.

White SM, Renda M, Nam NY, Klimatcheva E, Zhu Y, Fisk J, Halterman M, Rimel BJ, Federoff H, Pandya S, Rosenblatt JD, Planelles V (1999) Lentivirus vectors using human and simian immunodeficiency virus elements. J Virol 73: 2832-2840.

Wiznerowicz M, Trono D (2003) Conditional suppression of cellular genes: lentivirus vector-mediated drug-inducible RNA interference. J Virol 77: 8957-8961.

Wiznerowicz M, Trono D (2005) Harnessing HIV for therapy, basic research and biotechnology. Trends Biotechnol 23: 42-47.

Wolf D, Goff SP (2008) Host restriction factors blocking retroviral replication. Annu Rev Genet 42: 143-163.

Wong LF, Azzouz M, Walmsley LE, Askham Z, Wilkes FJ, Mitrophanous KA, Kingsman SM, Mazarakis ND (2004) Transduction patterns of pseudotyped lentiviral vectors in the nervous system. Mol Ther 9: 101-111.

Woodward CL, Prakobwanakit S, Mosessian S, Chow SA (2009) Integrase interacts with nucleoporin NUP153 to mediate the nuclear import of human immunodeficiency virus type 1. J Virol 83: 6522-6533.

Woolaway K, Asai K, Emili A, Cochrane A (2007) hnRNP E1 and E2 have distinct roles in modulating HIV-1 gene expression. Retrovirology 4: 28.

Wu X, Li Y, Crise B, Burgess SM (2003) Transcription start regions in the human genome are favored targets for MLV integration. Science 300: 1749-1751.
Wu X, Li Y, Crise B, Burgess SM, Munroe DJ (2005) Weak palindromic consensus sequences are a common feature found at the integration target sites of many retroviruses. J Virol 79: 5211-5214.

Wu X, Wakefield JK, Liu H, Xiao H, Kralovics R, Prchal JT, Kappes JC (2000) Development of a novel trans-lentiviral vector that affords predictable safety. Mol Ther 2: $47-55$.

Xia X, Zhang Y, Zieth CR, Zhang SC (2007) Transgenes delivered by lentiviral vector are suppressed in human embryonic stem cells in a promoter-dependent manner. Stem Cells Dev 16: 167-176.

Yamada K, McCarty DM, Madden VJ, Walsh CE (2003) Lentivirus vector purification using anion exchange HPLC leads to improved gene transfer. Biotechniques 34: 1074-1078, 1080.

Yamashita M, Emerman M (2004) Capsid is a dominant determinant of retrovirus infectivity in nondividing cells. J Virol 78: 5670-5678.

Yáñez-Muñoz RJ, Balaggan KS, MacNeil A, Howe SJ, Schmidt M, Smith AJ, Buch P, MacLaren RE, Anderson PN, Barker SE, Duran Y, Bartholomae C, von Kalle C, Heckenlively JR, Kinnon C, Ali RR, Thrasher AJ (2006) Effective gene therapy with nonintegrating lentiviral vectors. Nat Med 12: 348-353.

Yang H, Joo KI, Ziegler L, Wang P (2009) Cell type-specific targeting with surface-engineered lentiviral vectors codisplaying OKT3 antibody and fusogenic molecule. Pharm Res 26: 1432-1445.

Yang L, Bailey L, Baltimore D, Wang P (2006) Targeting lentiviral vectors to specific cell types in vivo. Proc Natl Acad Sci USA 103: 11479-11484.

Yang L, Yang H, Rideout K, Cho T, Joo KI, Ziegler L, Elliot A, Walls A, Yu D, Baltimore D, Wang P (2008b) Engineered lentivector targeting of dendritic cells for in vivo immunization. Nat Biotechnol 26: 326-334.

Yang S, Cohen CJ, Peng PD, Zhao Y, Cassard L, Yu Z, Zheng Z, Jones S, Restifo NP, Rosenberg SA, Morgan RA (2008c) Development of optimal bicistronic lentiviral vectors facilitates high-level TCR gene expression and robust tumor cell recognition. Gene Ther 15: 1411-1423.

Yang SH, Cheng PH, Sullivan RT, Thomas JW, Chan AW (2008a) Lentiviral integration preferences in transgenic mice. Genesis 46: 711-718.

Yang $\mathrm{SH}$, Cheng $\mathrm{PH}$, Banta $\mathrm{H}$, Piotrowska-Nitsche $\mathrm{K}$, Yang JJ, Cheng EC, Snyder B, Larkin K, Liu J, Orkin J, Fang ZH, Smith Y, Bachevalier J, Zola SM, Li SH, Li XJ, Chan AW (2008d) Towards a transgenic model of Huntington's disease in a non-human primate. Nature 453: 921-924.

Yi R, Bogerd HP, Cullen BR (2002) Recruitment of the Crm1 nuclear export factor is sufficient to induce cytoplasmic expression of incompletely spliced human immunodeficiency virus mRNAs. J Virol 76: 2036-2042.

Yilmaz A, Fernandez S, Lairmore MD, Boris-Lawrie K (2006) Coordinate enhancement of transgene transcription and translation in a lentiviral vector. Retrovirology 3: 13.

Yu D, Chen D, Chiu C, Razmazma B, Chow YH, Pang S (2001) Prostate-specific targeting using PSA promoterbased lentiviral vectors. Cancer Gene Ther 8: 628-635.

Yu J, McMahon AP (2006) Reproducible and inducible knockdown of gene expression in mice. Genesis 44: 252-261.

Yu SF, von Ruden T, Kantoff PW, Garber C, Seiberg M, Ruther U, Anderson WF, Wagner EF, Gilboa E (1986) Self-inactivating retroviral vectors designed for transfer 
of whole genes into mammalian cells. Proc Natl Acad Sci USA 83: 3194-3198.

Yu X, Yu Y, Liu B, Luo K, Kong W, Mao P, Yu XF (2003a) Induction of APOBEC $3 G$ ubiquitination and degradation by an HIV-1 Vif-Cul5-SCF complex. Science 302: 1056-1060.

Yu X, Zhan X, D'Costa J, Tanavde VM, Ye Z, Peng T, Malehorn MT, Yang X, Civin CI, Cheng L (2003b) Lentiviral vectors with two independent internal promoters transfer high-level expression of multiple transgenes to human hematopoietic stem-progenitor cells. Mol Ther 7: 827-838.

Yukl S, Pillai S, Li P, Chang K, Pasutti W, Ahlgren C, Havlir D, Strain M, Gunthard H, Richman D, Rice AP, Daar E, Little S, Wong JK (2009) Latently-infected CD4+ T cells are enriched for HIV-1 Tat variants with impaired transactivation activity. Virology 387: 98-108.

Zack JA, Arrigo SJ, Weitsman SR, Go AS, Haislip A, Chen IS (1990) HIV-1 entry into quiescent primary lymphocytes: molecular analysis reveals a labile, latent viral structure. Cell 61: 213-222.

Zaitseva L, Cherepanov P, Leyens L, Wilson SJ, Rasaiyaah J, Fassati A (2009) HIV-1 exploits importin 7 to maximize nuclear import of its DNA genome. Retrovirology 6: 11 .

Zaitseva L, Myers R, Fassati A (2006) tRNAs promote nuclear import of HIV-1 intracellular reverse transcription complexes. PLoS Biol 4: e332.

Zamborlini A, Lehmann-Che J, Clave E, Giron ML, TobalyTapiero J, Roingeard P, Emiliani S, Toubert A, de The H, Saib A (2007) Centrosomal pre-integration latency of HIV-1 in quiescent cells. Retrovirology 4: 63.

Zamule SM, Strom SC, Omiecinski CJ (2008) Preservation of hepatic phenotype in lentiviral-transduced primary human hepatocytes. Chem Biol Interact 173: 179-186.

Zanta-Boussif MA, Charrier S, Brice-Ouzet A, Martin S, Opolon P, Thrasher AJ, Hope TJ, Galy A (2009) Validation of a mutated PRE sequence allowing high and sustained transgene expression while abrogating WHV$X$ protein synthesis: application to the gene therapy of WAS. Gene Ther 16: 605-619.

Zennou V, Petit C, Guetard D, Nerhbass U, Montagnier L, Charneau P (2000) HIV-1 genome nuclear import is mediated by a central DNA flap. Cell 101: 173-185.

Zennou V, Serguera C, Sarkis C, Colin P, Perret E, Mallet J, Charneau P (2001) The HIV-1 DNA flap stimulates HIV vector-mediated cell transduction in the brain. Nat Biotechnol 19: 446-450.

Zhang B, Metharom P, Jullie H, Ellem KA, Cleghorn G, West MJ, Wei MQ (2004b) The significance of controlled conditions in lentiviral vector titration and in the use of multiplicity of infection (MOI) for predicting gene transfer events. Genet Vaccines Ther 2: 6.

Zhang B, Xia HQ, Cleghorn G, Gobe G, West M, Wei MQ (2001) A highly efficient and consistent method for harvesting large volumes of high-titre lentiviral vectors. Gene Ther 8: 1745-1751.

Zhang F, Thornhill SI, Howe SJ, Ulaganathan M, Schambach A, Sinclair J, Kinnon C, Gaspar HB, Antoniou M,
Thrasher AJ (2007b) Lentiviral vectors containing an enhancer-less ubiquitously acting chromatin opening element (UCOE) provide highly reproducible and stable transgene expression in hematopoietic cells. Blood 110: $1448-1457$.

Zhang J, Wang C, Ke N, Bliesath J, Chionis J, He QS, Li QX, Chatterton JE, Wong-Staal F, Zhou D (2007a) A more efficient RNAi inducible system for tight regulation of gene expression in mammalian cells and xenograft animals. RNA 13: 1375-1383.

Zhang W, Chen G, Niewiadomska AM, Xu R, Yu XF (2008) Distinct determinants in HIV-1 Vif and human APOBEC3 proteins are required for the suppression of diverse host anti-viral proteins. PLoS ONE 3: e3963.

Zhang X, Aida Y (2009) HIV-1 Vpr: a novel role in regulating RNA splicing. Curr HIV Res 7: 163-168.

Zhang XY, La Russa VF, Reiser J (2004a) Transduction of bone-marrow-derived mesenchymal stem cells by using lentivirus vectors pseudotyped with modified RD114 envelope glycoproteins. J Virol 78: 1219-1229.

Zhao J, Ma L, Wu YL, Wang P, Hu W, Pei G (1998) Chemokine receptor CCR5 functionally couples to inhibitory $\mathrm{G}$ proteins and undergoes desensitization. J Cell Biochem 71: 36-45.

Zheng JY, Chen D, Chan J, Yu D, Ko E, Pang S (2003) Regression of prostate cancer xenografts by a lentiviral vector specifically expressing diphtheria toxin A. Cancer Gene Ther 10: 764-770.

Zhou BY, Ye Z, Chen G, Gao ZP, Zhang YA, Cheng L (2007) Inducible and reversible transgene expression in human stem cells after efficient and stable gene transfer. Stem Cells 25: 779-789.

Zhu Y, Feuer G, Day SL, Wrzesinski S, Planelles V (2001b) Multigene lentiviral vectors based on differential splicing and translational control. Mol Ther 4: 375-382.

Zhu Y, Gelbard HA, Roshal M, Pursell S, Jamieson BD, Planelles V (2001a) Comparison of cell cycle arrest, transactivation, and apoptosis induced by the simian immunodeficiency virus SIVagm and human immunodeficiency virus type 1 vpr genes. J Virol 75: 37913801.

Zhu Y, Pe'ery T, Peng J, Ramanathan Y, Marshall N, Marshall T, Amendt B, Mathews MB, Price DH (1997) Transcription elongation factor P-TEFb is required for HIV1 tat transactivation in vitro. Genes Dev 11: 2622-2632.

Ziegler L, Yang L, Joo K, Yang H, Baltimore D, Wang P (2008) Targeting lentiviral vectors to antigen-specific immunoglobulins. Hum Gene Ther 19: 861-872.

Zufferey R, Donello JE, Trono D, Hope TJ (1999) Woodchuck hepatitis virus posttranscriptional regulatory element enhances expression of transgenes delivered by retroviral vectors. J Virol 73: 2886-2892.

Zufferey R, Dull T, Mandel RJ, Bukovsky A, Quiroz D, Naldini L, Trono D (1998) Self-inactivating lentivirus vector for safe and efficient in vivo gene delivery. $J \mathrm{Vi}$ rol 72: 9873-9880.

Zufferey R, Nagy D, Mandel RJ, Naldini L, Trono D (1997) Multiply attenuated lentiviral vector achieves efficient gene delivery in vivo. Nat Biotechnol 15: 871-875. 